\title{
SEISMIC ATTENUATION ANISOTROPY IN THE SOUTHERNMOST PART OF THE TAUPO VOLCANIC ZONE, NORTH ISLAND, NEW ZEALAND
}

\author{
by \\ Syuhada \\ A thesis submitted to \\ Victoria University of Wellington \\ in partial fulfillment of the requirements for the degree of \\ Master of Science \\ in Geophysics
}

School of Geography, Enviromental and Earth sciences

Victoria University of Wellington

March 2010 



\begin{abstract}
We investigate the mechanisms of seismic anisotropy and attenuation $(1 / Q)$ beneath the southernmost part of the Taupo Volcanic Zone (TVZ) by computing variations in S-wave attenuation factors with the direction of wave polarization. We rotate pairs of horizontal components in steps of $22.5^{\circ}$ from $0^{\circ}$ to $67.5^{\circ}$ and into the radial and transverse directions to search for the optimal separation of the attenuation curves and thereby determine an anisotropy symmetry system. The frequency dependence of $Q$ for the rotated S-waves is estimated by means of the non-parametric generalized inversion technique (GIT) of Castro et al. (1990) using shallow earthquakes ( $<40 \mathrm{~km}$ depth) recorded by GeoNet within $100 \mathrm{~km}$ of Mt. Ruapehu. To analyze the effects on computed attenuation properties of source locations, we divide our dataset into two groups: a "TVZ" group containing earthquakes within the TVZ in a distance range of 5-55 km and a "non-TVZ" group containing earthquakes outside the TVZ in a distance range of 5-50 km.

To measure $Q$, we compute the spectral amplitude decay with distance in terms of empirical functions at 20 separate frequencies in the frequency bands $2-10 \mathrm{~Hz}$ and $2-$ $12 \mathrm{~Hz}$ for the TVZ and non-TVZ datasets respectively. We construct homogeneous and two-layer $Q$ models for the TVZ dataset based on characteristic features of the attenuation function, while for outside TVZ we only analyse a homogeneous $Q$ model.

The homogeneous $Q$ models obtained for the two datasets indicate that $\mathrm{S}$-waves are more attenuated within the TVZ than outside. The homogeneous $Q$ model for the TVZ dataset reveals that the $\mathrm{S}$-wave is anisotropic at high frequencies $(f>6 \mathrm{~Hz})$ along N-S/EW directions with the relation $Q_{S_{E}}(f)=(6.15 \pm 1.22) f^{(1.73 \pm 0.12)}$ and $Q_{S_{N}}(f)=(4.14 \pm$ 1.26) $f^{(2.06 \pm 0.14)}$, while the non-TVZ dataset shows a weak frequency dependence of attenuation anisotropy at low frequencies in NE-SW/SE-NW directions giving the power law
\end{abstract}


function $Q_{S_{N E}}(f)=(50.93 \pm 1.18) f^{(0.20 \pm 0.10)}$ and $Q_{S_{S E}}(f)=(22.60 \pm 1.10) f^{(0.53 \pm 0.06)}$. Here, the uncertainty estimates are $95 \%$ confidence intervals.

To investigate the variation of attenuation anisotropy with depth within the TVZ, we first calculate $Q$ along propagation paths $(<25 \mathrm{~km}$, which corresponds to a maximum turning point depth of $9 \mathrm{~km}$ ) and then using paths of 25-55 km length. Small attenuation anisotropy with low attenuation in the $\mathrm{N}-\mathrm{S}$ direction for the upper crust of TVZ may be related to heterogenous structure as reported by previous studies. Attenuation anisotropy in the northwest direction yielding lower attenuation inferred for the deeper crust suggests the presence of connected melt aligned with the extension direction of TVZ . 


\section{ACKNOWLEDGMENTS}

Above all, thanks to the almighty God for everything. I would like to thank my advisors Prof. Martha Savage and Dr. John Townend for their useful personal advice and thoughtful discussions which provided much-needed guidance throughout this study.

Many thanks go to Dr. Raul Ramon Castro who explained to me the non-paramateric GIT method in the early stages of my work. Thank you also to Dr. Adrien Oth for providing me the bootstrap inversion script and for discussions during his summer holiday in Wellington.

Thank you to the New Zealand GeoNet project and it sponsors EQC, GNS Science and FRST for providing me with data used in this study. Also thanks to NZAID who provided me with a scholarship and gave me the opportunity to study in New Zealand. Thank you also to many staff and students at Victoria university of Wellington who have contributed to this study. Special thanks to Nicolas, Kathi, Sapi, Lloyd, Cherif, Abu, Sanjay and others for being very good friends of mine. Finally, I dedicate this work to someone who always loves me, supports and encourages me to overcome all kinds of difficulties during my study. 



\section{Contents}

Abstract

Acknowledgments $\quad$ v

Table of Contents vii

List of Figures $\quad$ ix

List of Tables

1 Introduction 1

1.1 Motivation . . . . . . . . . . . . . . . . 1

1.2 Relation between attenuation anisotropy and seismic velocity anisotropy . 3

1.3 Tectonic and geologic setting . . . . . . . . . . . . . . . 4

1.4 Thesis outline . . . . . . . . . . . . . . . . . . 9

2 Seismic attenuation anisotropy 11

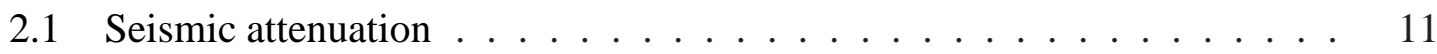

2.1.1 Scattering and intrinsic attenuation . . . . . . . . . . . 11

2.1.2 Geometrical spreading . . . . . . . . . . . . . 15

2.2 Measurement of seismic attenuation . . . . . . . . . . . . . . . . 16

2.2.1 Spectral ratio method . . . . . . . . . . . . . . . . 16

2.2.2 Spectral decay method ................. 17

2.2 .3 Coda-Q method . . . . . . . . . . . . . . . . . 17

2.2.4 Generalized inverse technique (GIT) . . . . . . . . . . . . 18

2.3 Seismic attenuation in volcanic regions . . . . . . . . . . . . . . . . . . 19

2.4 Seismic attenuation anisotropy . . . . . . . . . . . . 20

3 Methodology and data processing 23

3.1 The generalized inversion technique (GIT) . . . . . . . . . . . . 23

3.1.1 Nonparametric inversion method . . . . . . . . . . . . 25

3.1.2 Assessment of the inversion stability . . . . . . . . . . 31

3.2 Data . . . . . . . . . . . . . . . . . . 32

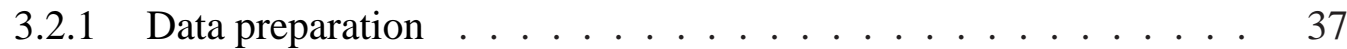

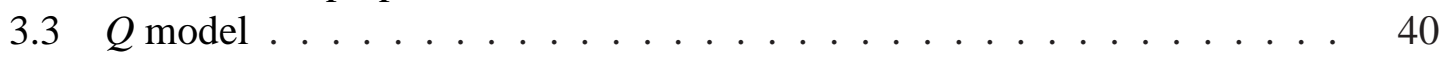

3.3.1 Homogenous $Q$ model . . . . . . . . . . . . . . . . 40

3.3.2 Two-layer $Q$ model . . . . . . . . . . . . . . . . . . 41 
4 Results $\quad 43$

4.1 Non-parametric attenuation function inside the TVZ . . . . . . . . . . 43

$4.2 Q$ model inside the TVZ . . . . . . . . . . . . . . . 50

4.2.1 Homogeneous $Q$ model . . . . . . . . . . . . . . 50

4.2.2 Two-layer $Q$ model . . . . . . . . . . . . . . . . . 53

4.3 Q model outside the TVZ . . . . . . . . . . . . . . . . 58

4.4 Source and directional site effects . . . . . . . . . . . . . . 62

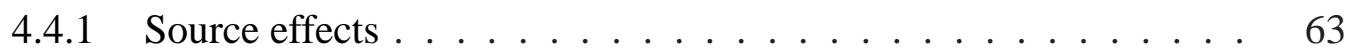

4.4.2 Directional site effects .................. 67

5 Discussion $\quad 79$

5.1 The data . . . . . . . . . . . . . . . . . . . 81

5.2 Inside the TVZ . . . . . . . . . . . . . . . . . . . . . 83

5.2.1 Amplitude decay functions .................... 83

5.2.2 $Q$ model . . . . . . . . . . . . . . . 84

5.3 Outside the TVZ . . . . . . . . . . . . . . . . . . . . . . . 88

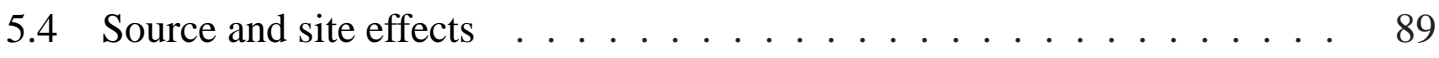

$\begin{array}{lll}6 & \text { Summary } & 93\end{array}$

$\begin{array}{ll}\text { References } & 95\end{array}$

$\begin{array}{ll}\text { A Event information } & 105\end{array}$

B Other decay curves 111

B.1 The TVZ decay functions . . . . . . . . . . . . . . . . . . . 112

B.2 The non-TVZ decay functions . . . . . . . . . . . 115

C Spectral ratio-differential attenuation method 119

C.1 Spectral ratio-differential attenuation method . . . . . . . . . . . . 119

C.2 Results . . . . . . . . . . . . . . . . 120 


\section{List of Figures}

1.1 The New Zealand tectonic setting _ . . . . . . . . . . . . . . . . . . 4

1.2 The TVZ tectonic setting . . . . . . . . . . . . . . 5

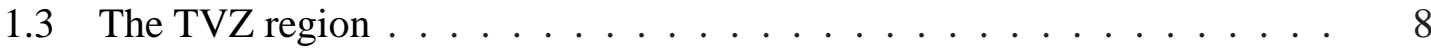

2.1 S-wave amplitude decay with time . . . . . . . . . . . . . . . . 13

2.2 S-wave amplitude decay with distance . . . . . . . . . . . . . . . . . 14

3.1 Application of weighting factors to the attenuation function . . . . . . . 27

3.2 The effect of bin width of the hypocentral distance to the decay curves . . 29

3.3 An example of a corrected attenuation function . . . . . . . . . 30

3.4 Comparison attenuation function with and without bootstrap inversion . . 31

3.5 Local earthquake distribution . . . . . . . . . . . . . . . . . . 34

3.6 Raypaths of the inside TVZ earthquakes . . . . . . . . . . . . . . 35

3.7 Depth cross section of the inside TVZ data . . . . . . . . . . . . 35

3.8 Raypaths of the outside TVZ earthquakes . . . . . . . . . . . . . 36

3.9 Depth cross section of the outside TVZ data . . . . . . . . . . . . . 36

3.10 A rotating reference frame of seismograms . . . . . . . . . . . . . 37

3.11 An example of smoothing spectra . . . . . . . . . . . . . 38

3.12 Examples of noise spectra . . . . . . . . . . . . 39

4.1 Amplitude decay function of E-W and N-S components . . . . . . . . 46

4.2 Attenuation function comparison . . . . . . . . . . . . . . . 47

4.3 Directional effect of amplitude decay function . . . . . . . . . . . . . . 48

4.4 Amplitude decay function of radial and transverse components . . . . . . 49

4.5 The direction of the $\mathrm{R}$ and $\mathrm{T}$ components of the TVZ data . . . . . . . . 50

4.6 The homogenous $\mathrm{Q}$ model inside the TVZ . . . . . . . . . . . 52

4.7 Ray tracing inside the TVZ . . . . . . . . . . . . . . . . . 54

4.8 The upper crust $Q$ model inside the TVZ . . . . . . . . . . . . . . 55

4.9 The lower crust $Q$ model inside the TVZ . . . . . . . . . . . . . . 56

4.10 The directions of the $\mathrm{R}$ and $\mathrm{T}$ components of the non-TVZ data . . . . . 58

4.11 Amplitude decay function of rotation $45^{\circ}$ (the non-TVZ) . . . . . . . 60

4.12 The $Q$ model outside the TVZ . . . . . . . . . . . . . . . . 61

4.13 Source functions . . . . . . . . . . . . . . . . . . 64

4.14 Site amplifications inside the TVZ $1 \ldots \ldots$. . . . . . . . . . . . . . . . . . . . 67

4.15 Site amplifications inside the TVZ $2 \ldots \ldots \ldots 6$

4.16 Site amplifications inside the TVZ $3 \ldots \ldots$. . . . . . . . . 69

4.17 Contours of the site amplifications of OTVZ and WTVZ station . . . . 71 
4.18 Contours of the site amplifications of PKVZ and WPVZ station . . . . . 72

4.19 Directional site effects around the TVZ region (the TVZ data) . . . . . 73

4.20 Site amplifications outside the TVZ $1 \ldots \ldots$. . . . . . . . . . . . . . . . . . . 74

4.21 Site amplifications outside the TVZ $2 \ldots \ldots \ldots$. . . . . . . 75

4.22 Site amplifications outside the TVZ $3 \ldots \ldots \ldots 76$

4.23 Directional site effects around the TVZ region (the non-TVZ data) . . . . 77

5.1 Theoretical amplitude decay . . . . . . . . . . . . . . . . . . 82

5.2 Comparison $Q$ model . . . . . . . . . . . . . . . . . . . . . . . . . . . 84

5.3 Comparison of average $Q$ model $\ldots \ldots \ldots$. . . . . . . . . 86

B.1 Amplitude decay function of rotation $22.5^{\circ}(\mathrm{TVZ}) \ldots \ldots \ldots$

B.2 Amplitude decay function of rotation $45^{\circ}$ (TVZ) . . . . . . . . . . . . . . 113

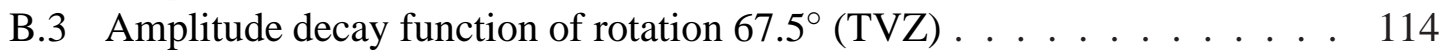

B.4 Amplitude decay function of $\mathrm{E}$ and $\mathrm{N}$ components (non-TVZ) . . . . . . 115

B.5 Amplitude decay function of R and T components (non-TVZ) . . . . . 116

B.6 Amplitude decay function of rotation $22.5^{\circ}$ (non-TVZ) . . . . . . . . 117

B.7 Amplitude decay function of rotation $67.5^{\circ}$ (non-TVZ) $\ldots \ldots . . . . .118$

C.1 Spectral ratio method . . . . . . . . . . . . . . . . 122 


\section{List of Tables}

2.1 Typical $Q$ values of rocks . . . . . . . . . . . . . . . . . . . 12

$2.2 Q_{0}$ and $\alpha$ in different tectonic regions $\ldots \ldots \ldots \ldots$

3.1 Locations of the GeoNet stations used for analysis $\ldots \ldots \ldots$

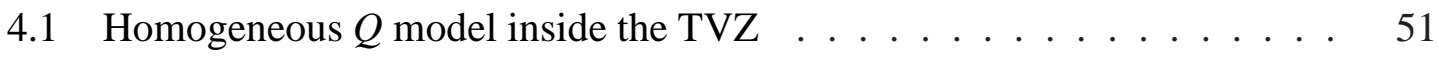

4.2 Raytracing velocity models for S-waves in the southernmost part of the TVZ 53

4.3 Upper crust $Q$ model inside the TVZ . . . . . . . . . . . . . . . . 54

4.4 Lower crust $Q$ model inside the TVZ . . . . . . . . . . . . . . . . 57

4.5 Homogeneous $Q$ model outside the TVZ . . . . . . . . . . . . . 59

4.6 Number of earthquakes recorded by GeoNet stations around the TVZ . . 62

4.7 Examples of source parameters . . . . . . . . . . . . . . . 65

A.1 Events used for Q model of inside TVZ . . . . . . . . . . . 105

A.2 Event used for Q model of outside TVZ . . . . . . . . . . . . 107 


\section{Chapter 1}

\section{Introduction}

\subsection{Motivation}

Seismic attenuation occurs when seismic waves passing through the Earth lose their energy in different amounts due to geometric spreading, scattering, multipathing and intrinsic processes (e.g. Stein and Wysession, 2003). The amount of attenuation is usually param-

eterised by the inverse of the quality factor $Q$, namely $Q^{-1}$. The two main mechanisms thought to be responsible for this amplitude variation are scattering and intrinsic attenuation. Intrinsic attenuation converts the seismic energy into heat, while scattering causes redirection of the energy of the wave (e.g. Stein and Wysession, 2003). In volcanic regions, both intrinsic and scattering attenuation are important but it is difficult to distinguish the mechanism of predominant attenuation (Arevalo et al., 2003).

Seismic attenuation measurements have been conducted on several volcanoes and gave significant contributions to both geological and geophysical studies in volcanic regions. For example, the seismic attenuation structure at Rabaul volcano, Papua New Guinea, has been studied using regional earthquake data and the spectral ratio method to estimate the quality factors of $\mathrm{P}$ and $\mathrm{S}$ waves (Gudmundsson et al., 2004). Gudmundsson et al. discovered that near-surface rocks inside the caldera are relatively more attenuative than those outside the caldera, with $Q$ values ranging from 15-20 in the caldera indicating the 
influence of fractured rocks and the presence of geothermal fluids. Del Pezzo et al. (2006) estimated the attenuation factor on Mt. Vesuvius using S-coda envelopes and determined the minimum value of the total quality factor $(Q)$ around 10 at $2 \mathrm{~Hz}$. The attenuation at Deception Island volcano (Antarctica) was studied by Arevalo et al. (2003), who analysed P-waves, S-waves and coda waves using frequency-dependent and -independent methods to determine the quality factor. Those results indicate that the region has relatively high attenuation compared to other volcanic or tectonic regions.

In an anisotropic medium, the attenuation depends on the propagation direction because scatters may be preferentially aligned. Recently, several techniques have been broadly adopted in seismic attenuation anisotropy studies to reveal the physical properties of the Earth and to separate the site and attenuation terms from the source term. Carter and Kendall (2006) used the spectral ratio method to quantify the differential attenuation of the slow waves $\left(S_{1}\right)$ and fast waves $\left(S_{2}\right)$ using a microseismic dataset extracted from an active oil well in Valhall, a North sea oil field. The result of the measurements appeared to contradict Hudson's theory (Hudson, 1981), which predicts that the slower arrival S2 should be more attenuated at high frequencies than the faster arrival $S 1$. Using a method based on the spectral ratio technique and a waveform-fitting method for the 1999 Chi-chi earthquake sequence, Liu et al. (2005) calculated $Q$ value of the near-surface crust to be approximately 61-68 for the fast components and 43-52 for the slow components in the range frequency of $2-15 \mathrm{~Hz}$. This observation suggests the presence of microcrack alignment, which is also suggested as the main cause of the velocity anisotropy in the same region.

An empirical method using a generalized inversion technique (GIT) is another common way of quantifying the anisotropy of body wave attenuation and to separate the site and attenuation terms from the source function (e.g. Castro et al., 1999). Castro et al. attempted to identify the presence of seismic anisotropy in the Marche region of Italy. That study provided additional evidence of seismic anisotropy in the region, as previously reported based on seismic velocity anisotropy observations (e.g. Margheriti et al., 1996). The low $Q_{S}$ anisotropy estimation using deep wave paths $(55-125 \mathrm{~km})$ showed that the $Q_{S V}$ val- 
ues are greater than the $Q_{S H}$ values at low frequencies $(f<4 \mathrm{~Hz})$, suggesting magmatic activity related to the compressional tectonics in that region.

\subsection{Relation between attenuation anisotropy and seismic velocity anisotropy}

In an anisotropic medium, S-waves split into two orthogonally-polarized fast and slow directions causing a difference in travel times (delay times). Several techniques can be used to determine the delay time, which separates the fast $\left(\mathrm{S}_{1}\right)$ and slow $\left(\mathrm{S}_{2}\right)$ components, and the direction of the fast shear wave-component (e.g. Savage, 1999). The splitting time is a function of the thickness of the anisotropic layer and the magnitude of the anisotropy, whereas the fast polarization direction commonly provides information on the orientation of the anisotropy axes (Barton, 2007). Seismic anisotropy can be caused either by aligned cracks or crystal lattice-preferred orientation (e.g. Stein and Wysession, 2003). Unfortunately, seismic velocity anisotropy analysis alone cannot determine the likely cause of the anisotropy, since aligned cracks and lattice-preferred orientation may yield the same observed seismic velocity anisotropy. However, these two mechanisms should produce different observations of seismic attenuation anisotropy. In particular, attenuation anisotropy may be strongly produced by preferential alignment of cracks but not by crystal lattice orientation (Carter and Kendall, 2006). Therefore, using a combination of velocity anisotropy and attenuation anisotropy measurements may allow us to determine the possible mechanism of the anisotropy and thereby elucidate the structure and composition of the Earth.

This study aims to measure attenuation anisotropy in the southernmost part of the TVZ and to test its conformity with the previously measured seismic anisotropy. This research is also motivated by the fact that there are no seismic attenuation anisotropy studies in New Zealand and we need to better understand anisotropic phenomena in the volcanic region, since seismic velocity anisotropy alone cannot distinguish possible causes of anisotropy. 


\subsection{Tectonic and geologic setting}

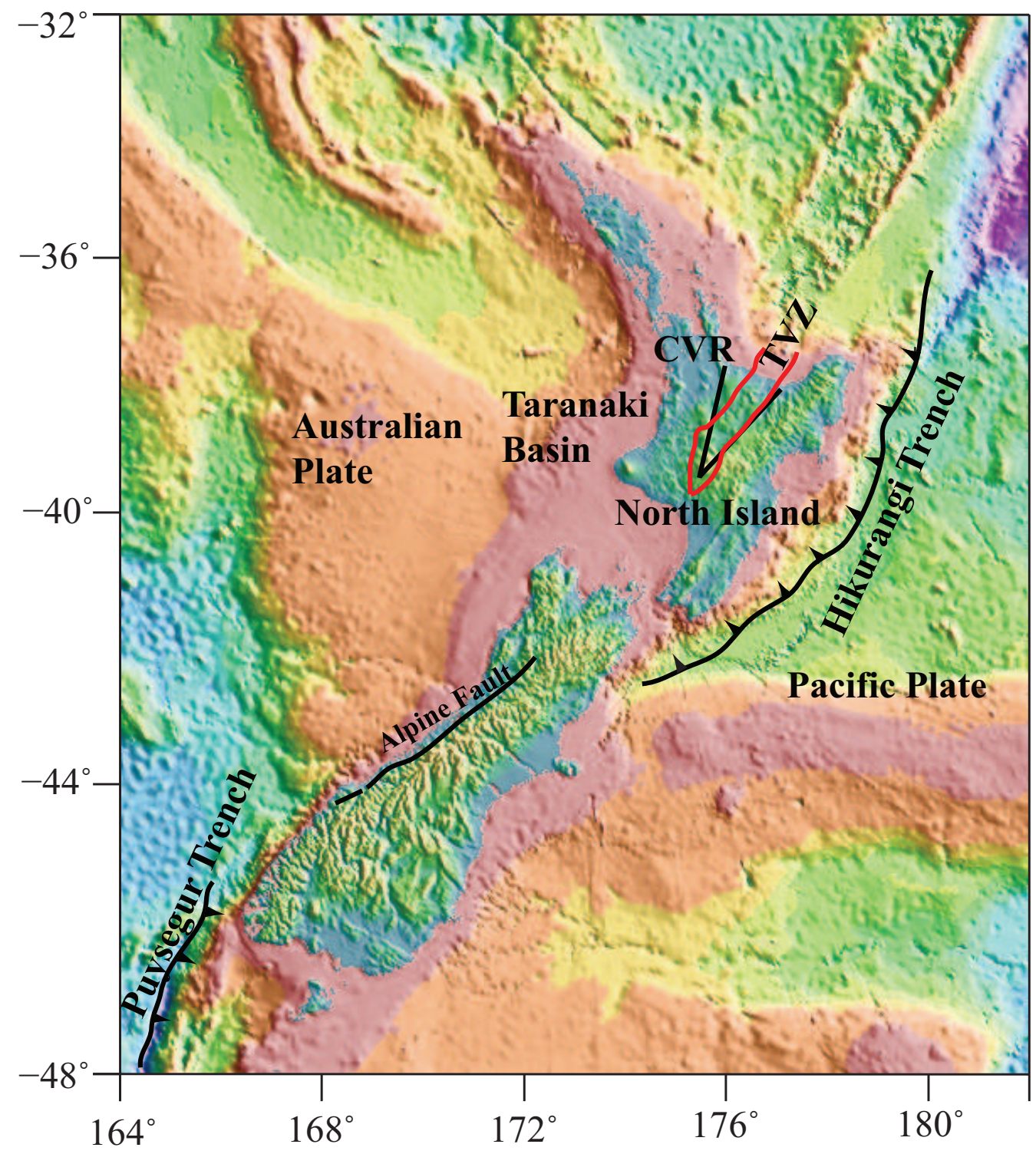

Figure 1.1 The plate tectonic setting of New Zealand and its environs describing the interaction between the Pacific and the Australian plates (adapted after Smith and Sandwell (1997), http://topex.ucsd.edu/marine_topo/mar_topo.html). 
New Zealand is located at the boundary between the Pacific and the Australian plates. To the southwest of New Zealand, the oceanic crust of the Australian plate is subducting beneath continental crust of the Pacific plate at the Puysegur trench (Lamarche and Lebrun, 2000). Along the Hikurangi margin of the North island, the thin dense Pacific plate subducts obliquely beneath the thicker lighter continental crust of the Australian plate with a relative convergence rate of $43 \mathrm{~mm} /$ year (Beavan et al., 2002) (Figure 1.1). These two subduction margins are connected by a northeast transpressional slip movement along the Alpine fault which crosses New Zealand (Sutherland et al., 2006).

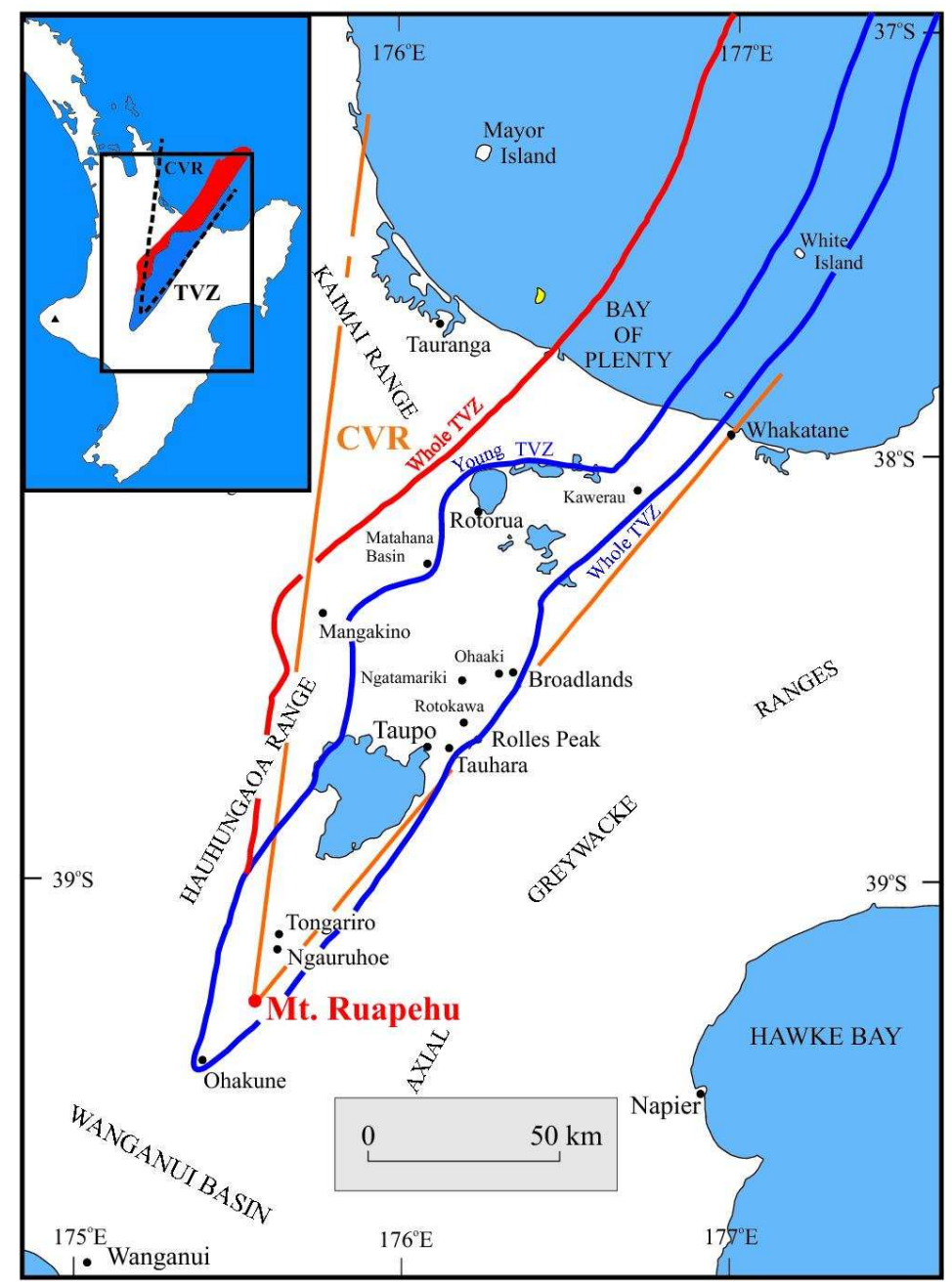

Figure 1.2 The tectonic setting of the North island of New Zealand illustrates the boundary of the TVZ extending from Mt. Ruapehu in the southern part to White Island in the northern part (Adapted after Wilson et al. (1995))

The Central Volcanic Region (CVR) is a wedge-shaped basin characterized by mate- 
rial with low density and seismic velocities (e.g. Stratford and Stern, 2006). The CVR is the continuation of an oceanic back-arc basin behind the Tonga Kermadec Trench, the Lau Havre Trough, into continental New Zealand (Stern et al., 2010). Shallow $(<6$ $\mathrm{km}$ depth) and subduction zone earthquakes dominate seismicity within the CVR (e.g. Ansell and Bannister, 1996). 1.8-2.7 km thick volcanic deposits cover the surface of the CVR (Stern and Davey, 1987). The eastern part of the CVR is defined by a volcanically active region as discussed below.

Oblique subduction in the northern part of New Zealand results in a region of rapid extension with extremely active volcanism and high geothermal activity (Wilson et al., 1995). The most active part in this region is the Taupo Volcanic Zone (TVZ) which can be defined as the young active volcanic and high heat flow region extending from Mt. Ruapehu to White Island (e.g. Wilson et al., 1995) (Figure 1.2). The TVZ is a region under active extension with an extension rate of up to $15 \mathrm{~mm} \mathrm{yr}^{-1}$ at the Bay of Plenty, and decreasing to $<5 \mathrm{~mm} \mathrm{yr}^{-1}$ near the southernmost part of the TVZ (Eberhart-Phillips and Reyners, 2009; Stern, 2009). Andesitic volcanic activity in the TVZ started around 2 Ma (e.g. Wilson et al., 1995). Intensive and large rhyolitic activity began from around 1.6 Ma and it produced the largest and most vigorous rhyolitic magmatic regime on Earth; it is about $300 \mathrm{~km}$ long (including $200 \mathrm{~km}$ inland), more than $60 \mathrm{~km}$ wide and at least $10,000 \mathrm{~km}^{3}$ of rhyolitic magma has been erupted (Wilson et al., 1995; Houghton et al., 1995). The central $6000 \mathrm{~km}^{2}$ of the TVZ produces an average heat flux of $700 \mathrm{~mW} / \mathrm{m}^{2}$ (Bibby et al., 1995). A migration and $30^{\circ}$ clockwise rotation of the volcanic front since 4 Ma defined the eastern boundary of the TVZ (Stern et al., 2006).

The southernmost part of the TVZ is a volcanic area dominated by NNE-SSW trending faults parallel to the orientation of volcanic vents. These faults are probably related to magmatic intrusion into shallow $(<10 \mathrm{~km})$ crustal reservoirs and overlying dike injection (Cole, 1990). This area is called the Tongariro Volcanic Center, consisting of Mt. Ruapehu, Mt. Ngauruhoe and Mt. Tongariro (Fig. 1.3).

Mt. Ruapehu is an active andesitic volcano located at the southern terminus of the 
TVZ. It currently reaches $2979 \mathrm{~m}$ in elevation and is estimated to have a volume of 110 $\mathrm{km}^{3}$ (Hackett, 1985). Active faults surrounding Mt. Ruapehu are formed as a result of subsidence in the central axis of the TVZ and define the TVZ's boundary in the southern part of the volcano (Cole, 1990) (Fig. 1.3).

There are four major formations around Mt. Ruapehu: Te Herenga (250-180 ka), Wahianoa (160-115 ka), Mangawhero (55-15 ka), and Whakapapa (<15 ka) (Gamble et al., 2003). Gamble et al. (2003) suggest that the maximum age for the inception of volcanism at Ruapehu is around $340 \mathrm{ka}$ and the average magma flux at Mt. Ruapehu is 0.6$1.2 \mathrm{~km}^{3} / \mathrm{ka}$. The volcanic deposits around Ruapheu, which are mostly avalanche debris and lahar deposits, appear as a low-velocity volume $(V p<4 \mathrm{~km} / \mathrm{s})$ in the seismic tomography image (Rowlands et al., 2005).

The eruptions of Ruapehu started more than 120,000 years ago and the evidence concerning the history of the eruptions is recorded in the sequence of tephra layers within its surrounding ring plain (Donoghue et al., 1995). The 1995/1996 eruption of Mt. Ruapehu provided further valuable insight into geological and geophysical properties around the volcano. Bryan and Sherburn (1999) analysed the seismicity related to the eruption sequence in 1995-1996 and suggested that it reflects processes connected to the intrusion of magma to shallow depth and changes in the volcanic plumbing system. The characteristics of seismicity associated with the 1995-1996 eruptions were different from those in the period 1971-1995. Specifically, volcanic tremor and volcanic earthquakes were dominated by a frequency of $2 \mathrm{~Hz}$ before the eruptions and became more broadband during the 1995-1996 eruptions (Sherburn et al., 1999). Sherburn et al. suggested that the types of seismicity and the eruption styles may have been affected by the presence of the crater lake at Ruapehu. They also hypothesized that the volcanic plumbing configuration would slowly return to the pre-eruptive state and the seismicity characteristics return to those observed in the pre-eruptive period.

In volcanic regions, the geophysical and geochemical properties of the crustal structure may change due to volcanic activities. When an eruption occurs, the magma ascends 


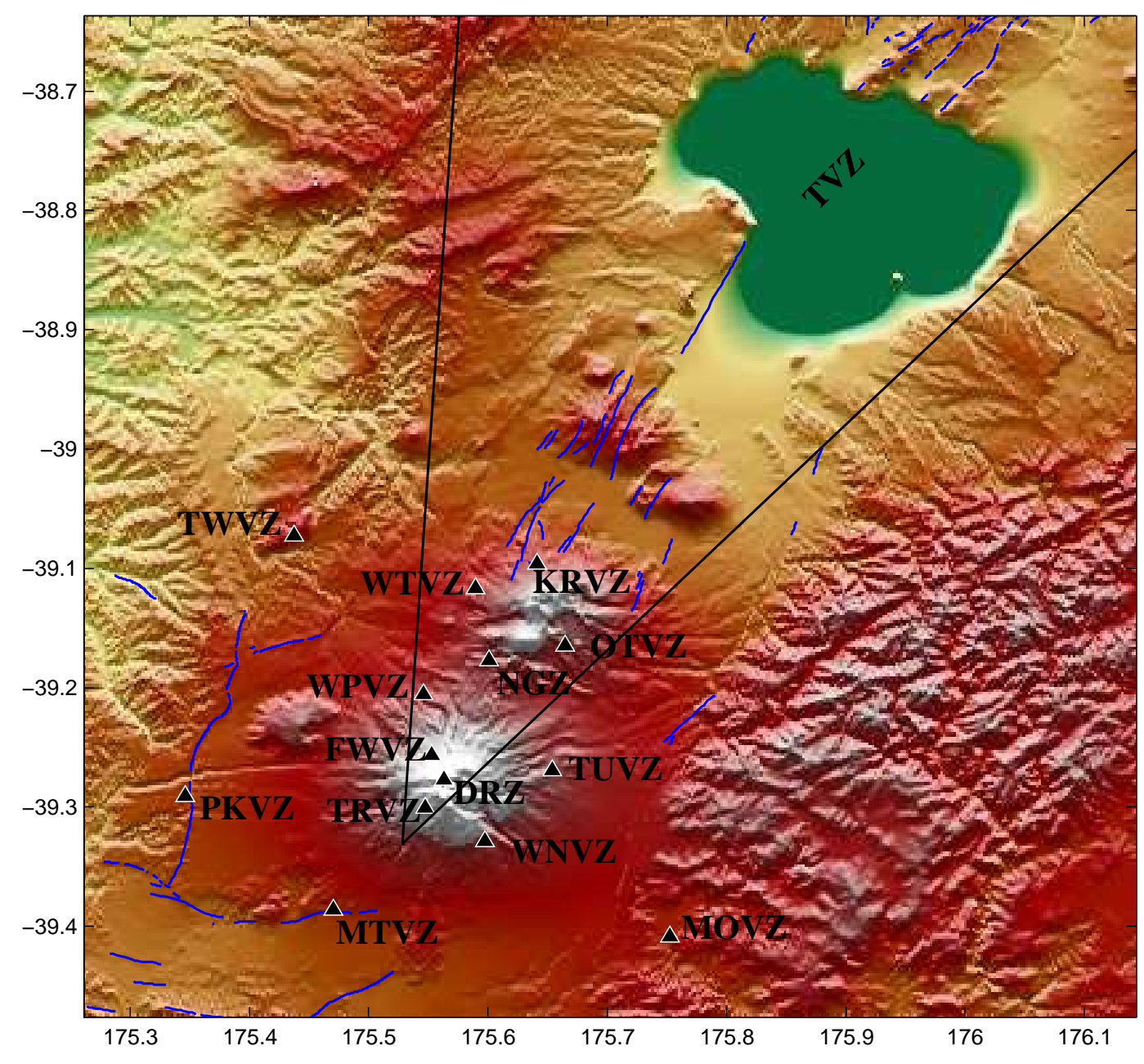

Figure 1.3 The southernmost part of the TVZ and Mt. Ruapehu showing the GeoNet stations (the black triangles) used in this study. The blue lines represent active faults around the TVZ (Cole, 1990).

to the surface causing changes in stress, pore pressure and temperature of the volcanic rocks. These changes affect the crack orientations, influencing the propagation of seismic waves (Nur and Simmons, 1969). Recent studies of seismic anisotropy of Ruapehu using the shear-wave splitting method show changes in orientation of crustal anisotropy by $80^{\circ}$, interpreted as being caused by reoriented cracks after the 1995-1996 eruptions (Miller and Savage, 2001; Gerst and Savage, 2004). These studies suggest that the changes were due to repeated filling or depressurizing of a magmatic dyke system, resulting in the reoriented cracks. The presence of cracks also influences the attenuation of seismic waves. Titzschkau et al. (2009) demonstrated temporal changes of relative P wave attenuation connected with the presumed changes in stress affecting seismic anisotropy. 
Titzschkau et al. (2009) further showed that coda wave attenuation is strongly frequencydependent with small fluctuations over the entire time period of measurement and has a relation of $Q_{c}=(54 \pm 7) f^{(1.02 \pm 0.06)}$. The estimation of direct P- and S-wave attenuation also revealed high attenuation values at the summit of Ruapehu volcano, indicating the presence of shallow highly scattering layers in the volcanic deposits. Recent attenuation tomography studies show a relatively low $Q_{P}(<150)$ beneath the central part of the TVZ at depths of $50-85 \mathrm{~km}$, suggesting that there is a strong connection between volcanism and low attenuation in the shallow mantle and crust (Eberhart-Phillips et al., 2008). Eberhart-Phillips et al. (2008) suggested that the strong relation observed between low attenuation of $\mathrm{P}$-waves, low $\mathrm{V}_{P}$ and high $\mathrm{V}_{P} / \mathrm{V}_{S}$ is mainly governed by high temperature in this part of shallow crust related to partial melt in dikes and veins (e.g. Reyners et al., 2006).

\subsection{Thesis outline}

The remainder of this thesis is divided into five main chapters. The fundamental seismological concepts addressed during this project are introduced in Chapter 2, which starts with the theoretical background of seismic attenuation, seismic attenuation in volcano areas, and seismic attenuation anisotropy observed in other regions. The method and steps of the data processing including error assessment of the measurements are presented in Chapter 3. The characteristics of the data used in the inversion are also described in this chapter. Chapter 4 describes the implementation of the general inverse technique described in the previous chapter and its application to our dataset. Then, the attenuation function is simultaneously separated from the source and site terms to yield the $Q$ anisotropy model. The implications of the attenuation anisotropy model and the source and directional site effects for the regional seismotectonics of the southernmost part of the TVZ are discussed in Chapter 5. Finally, Chapter 6 is a summary of the main results and conclusions of the thesis. 


\section{Chapter 2}

\section{Seismic attenuation anisotropy}

\subsection{Seismic attenuation}

Attenuation is an important characteristic of seismic propagation used to quantify and characterize the properties and ambient conditions in the Earth. Seismic attenuation can be described as the amplitude decay of seismic waves with time and distance due to anelastic and elastic processes as the waves propagate through the Earth. In anelastic processes the energy of a propagating wave is not conserved as the seismic energy is converted into heat. In contrast, the amplitude decay of seismic waves in response to elastic processes including geometrical spreading and scattering satisfies the law of conservation of energy within the waves themselves.

\subsubsection{Scattering and intrinsic attenuation}

Many factors can produce attenuation of body waves and not all seismic attenuation mechanisms are well understood (e.g. Stein and Wysession, 2003). The most important mechanisms governing attenuation are scattering and intrinsic absorption. In the scattering case, the energy of the seismic waves is reradiated in different directions or with different polarizations when the seismic waves encounter nonuniform media such as cracks or other lateral velocity variations. Intrinsic attenuation within the Earth occurs when the seismic 
energy is converted into heat due to internal friction such as friction along grain boundaries (Stein and Wysession, 2003).

Attenuation effects are usually expressed by the dimensionless quantity $Q$ (quality factor). $Q$ is measured in terms of the fractional energy loss per cycle as:

$$
\frac{1}{Q(\omega)}=-\frac{1}{2 \pi} \frac{\Delta E}{E}
$$

Where $E$ is the energy of the propagating seismic waves, $\Delta E$ is the energy lost per cycle, and $Q$ is the quality factor (Stein and Wysession, 2003). $Q$ represents the energy decay of an oscillation as a function of distance, whereas the physical property of the system causing attenuation on seismic waves is generally parameterized in terms of $Q^{-1}$. The value of $Q$ is observed to vary with the geological setting: for example, the presence of magma and cracks cause volcanic regions commonly to have lower $Q$ values than those in other tectonic regions (e.g. Del Pezzo et al., 1985; Arevalo et al., 2003). The $Q$ values are also different for different rock types. Table 2.1 shows the ranges of the $Q$ values for different rock types.

Table 2.1 Typical $Q$ values of rocks. The Q values for P-waves are usually to be two or three times higher than those for S-waves. (Sheriff and Geldart, 1995)

\begin{tabular}{ll}
\hline \hline Rock type & $Q$ \\
\hline Sedimentary rocks & $20-200$ \\
Sandstone & $70-130$ \\
Shale & $20-70$ \\
Limestone & $50-200$ \\
Chalk & 135 \\
Dolomite & 190 \\
Rocks with fluids in pore space & $5-50$ \\
Metamorphic rocks & $200-400$ \\
Igneous rocks & $75-300$ \\
\hline
\end{tabular}


It is generally difficult to determine $Q$ directly from the definition given in equation 2.1, since most circumstances do not generate a medium with stress waves of unchanging amplitude and period (Aki and Richards, 1980). Nevertheless, there are some conditions under which attenuation can be directly observed (for illustration see Figure 2.1 and 2.2):

1. the temporal decay of amplitude at fixed wavenumber;

2. the spatial decay in a propagating wave at fixed frequency.

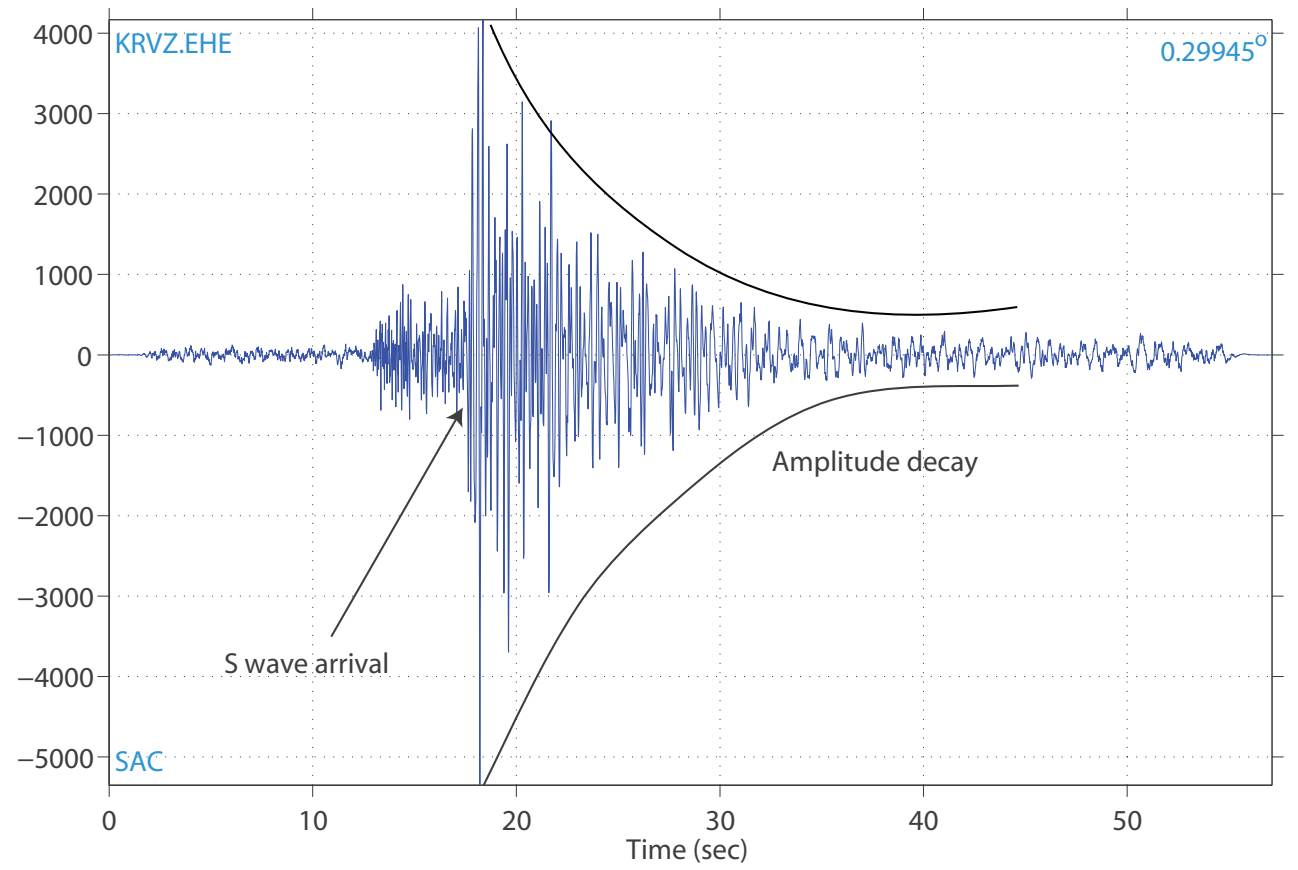

Figure 2.1 Seismogram from an earthquake in the southernmost part of the TVZ showing how the S-wave amplitude decays with increasing lapse time (time after the earthquake's origin time).

By assuming that attenuation is a linear phenomenon and that it is approximately linearly dependent on frequency, Fourier analysis can be applied to get the correct effect of attenuation on actual seismic signals (e.g. Feustel, 1998). For a medium with a linear stress-strain relationship under condition (1) or (2), the seismic wave's amplitude $A$ is proportional to the square root of the energy $\left(E^{1 / 2}\right)$. Further assuming that $Q>>1$ implies that successive peaks have almost the same strain energy (the energy stored within material as a result of applying a force to an elastic material (Stein and Wysession, 2003). It is 

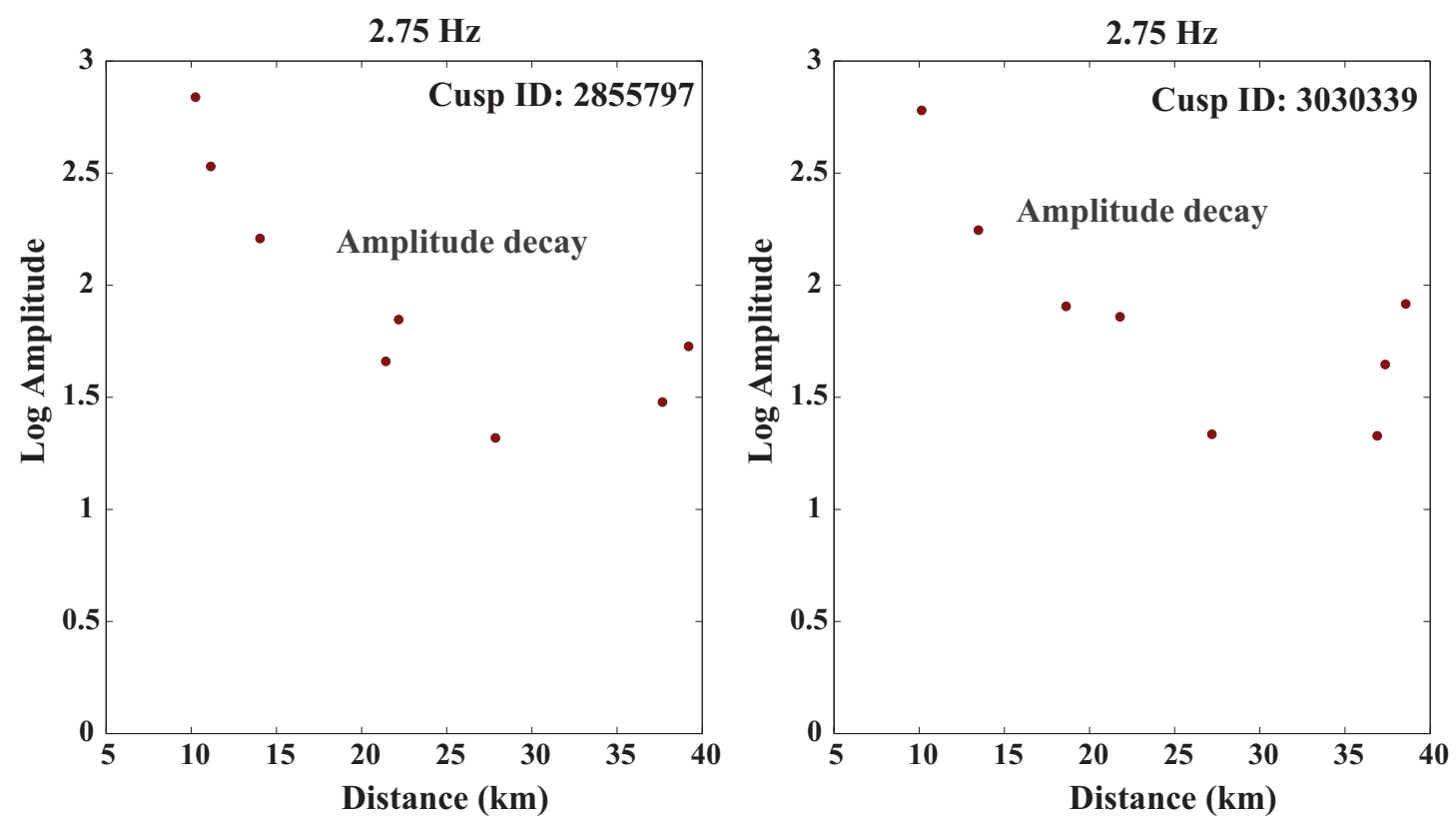

Figure 2.2 Examples of the observed amplitudes of S-wave for two different events at the same frequency recorded by GeoNet stations at Mt. Ruapehu, showing amplitude decay due to attenuation as the distance between the source and receiver increases.

important to note here that the amplitude $A$ may represent a maximum particle velocity or a stress component in the wave. Therefore, that condition can be expressed mathematically as:

$$
\frac{1}{Q(\omega)}=-\frac{1}{\pi} \frac{\Delta A}{A}
$$

From equation 2.2, we can describe the amplitude fluctuations due to attenuation in terms of two different mathematical models corresponding to the two different situations, the temporal and spatial decay, as follows (for detailed derivation of the equations, see Aki and Richards (1980)) :

$$
\begin{gathered}
A(t)=A_{0} \exp \left[-\frac{\omega t}{2 Q}\right] \\
A(x)=A_{0} \exp \left[-\frac{\omega x}{2 v Q}\right]
\end{gathered}
$$

Here, $A(t)$ and $A(x)$ are the seismic wave amplitudes after propagating for a time $t$ and distance $x$, respectively. $A_{0}$ is the initial amplitude of the seismic wave, $\omega$ is the angular 
frequency and $v$ is the phase velocity. Aki and Richards (1980) emphasized that geometrical spreading effects due to material heterogeneity also have an important role to play in any spatial decay, so their contribution cannot be neglected.

The value of $Q$ is commonly constant for frequencies less than $1 \mathrm{~Hz}$ (Aki and Richards, 1980). At higher frequencies, attenuation does depend on frequency and is often represented using a power law relation (Aki, 1980a):

$$
Q(f)=Q_{0}\left[\frac{f}{f_{0}}\right]^{\alpha}
$$

where $f_{0}$ is the reference frequency, $Q_{0}$ is the $Q$ value at this reference frequency and $\alpha$ is the frequency-dependency coefficient. The mechanism of frequency-dependent attenuation implied by equation 2.5 is not well understood, although numerous attenuation studies of body, surface and coda waves documented this phenomenon (e.g. Aki, 1980a; Kinoshita, 1994; Giampiccolo et al., 2007). Aki (1980b) observed that strong frequency dependence (high $\alpha$ ) is caused by the presence of high density cracks and faults increasing body waves scattering, while the presence of hot materials characterized by weak frequency dependence (low $\alpha$ ) suggests a dominance of intrinsic mechanism over scattering attenuation.

\subsubsection{Geometrical spreading}

It is well known that geometrical spreading causes seismic wave amplitudes to decay with distance (e.g. Castro et al., 1990), which is a consequence of the spreading of seismic energy when waves and wave fronts expand in a larger volume area. This mechanism is independent of frequency and has an obvious and significant contribution to amplitude decay. In a homogenous medium and at short distances, the standard function commonly used to describe geometrical spreading of a body wave can be described as

$$
G(R)=\frac{1}{R}
$$

The phenomenon of geometric spreading in complex media is difficult to model (Stein and Wysession, 2003) and may lead to different forms of the geometrical spreading function in the real Earth (e.g. Castro et al., 1999). 


\subsection{Measurement of seismic attenuation}

The principal goal in the determination of an attenuation model is to separate the source and site terms from the recorded seismograms. Several techniques have been developed to achieve that objective (e.g. Feustel, 1998). Those techniques are able to quantify the quality factor $(Q)$ based on an attenuation function as a function of time or distance. Although the methods have proven effective and accurate in measuring $Q$, the most significant problem is to choose an appropriate method to measure attenuation specific to site requirements (Feustel, 1998). All the methods have their individual advantages and disadvantages and many geophysicists have contributed to their development. Methods that are commonly used to compute attenuation models from recorded seismograms are described below (after Feustel, 1998):

\subsubsection{Spectral ratio method}

This method considers the ratio between a body wave's spectrum at a station of interest and the spectrum at a reference station or the spectrum of another part of the seismogram (Toksoz and Johnston, 1981). This method yields a single path-average $Q$, so it can be applied to datasets containing only a few ray-paths and sources. In addition, this method effectively cancels the source, the site and the instrument response effects and yields an attenuation model relative to the seismogram recorded at the reference station or reference phase (e.g. Carter and Kendall, 2006; Liu et al., 2005). For this reason, it usually requires a particular array geometry in the dataset (e.g. homogenous dataset containing only events recorded by all seismic stations). The method is based on the fact that higher frequencies of seismic spectra attenuate faster than lower frequencies. This method only measures the quality factors of the direct body wave, which is independent of frequency and cannot distinguish attenuation due to intrinsic and scattering processes. Therefore, when implementing the spectral ratio method, only a small window of the signal can be analyzed to ensure that there is no contamination from scattered energy in the coda waves. 


\subsubsection{Spectral decay method}

The spectral decay method is based on Brune's (1970) source model assuming that unattenuated displacement spectra amplitude for all frequencies below the corner frequency are flat, while frequencies past the corner frequency are well characterized by a displacement spectra slope of $-2\left(\omega^{-2}\right)$. $Q$ measurement using this method is performed by estimating the slope of the signal spectrum over a frequency band limit. Then, the estimated slope is compared to the $\omega^{-2}$ slope of Brune's model. As with the spectral ratio method, the $Q$ term resulting from this method of analysis is independent of frequency and valid for a single path of direct body waves. Since this method uses Brune's model as a reference signal, it does not need specialized array geometry or sources. However, the accuracy of the method is very sensitive to the presence of scattered wave energy. The contamination of signal scattering in the signal window can raise the signal energy affecting the $Q$ measurement (Feustel, 1998). It is also requires knowledge or assumptions regarding the source mechanism.

\subsubsection{Coda-Q method}

The method measures $Q$ from the decay rate of coda waves with increasing lapse time (measured from the origin time of the seismic event). This method assumes that coda waves consist of body waves scattered from random heterogeneities in the Earth (Aki and Chouet, 1975). Therefore, an interpretation of the $Q$ model obtained using this method is difficult because the decay of coda energy involves both scattering and intrinsic absorption effects. However, a modification of the method is capable of separating intrinsic and scattering attenuation, which may help infer the dominant attenuation (e.g. Del Pezzo et al., 2006). The $Q$ coda can be estimated as a function of frequency and time. A theoretical model for the distribution of coda wave energy proposed by Sato (1977) shows that the decay rate of coda wave amplitude is independent of both the ray path and the sourcereceiver distance (Feustel, 1998). The time window for the $Q$ coda analysis must be picked 
carefully because the resulting $Q$ model analyzed may be dependent on the lapse time and sensitive to the contributions of multiply-scattered waves. Although it is possible to calculate $Q$ coda for a single path, it is better to measure $Q$ coda for several event-station pairs to reveal the $Q$ coda in a particular area. In addition, as with the spectral decay method, specialized array geometry and active sources are not considered essential.

\subsubsection{Generalized inverse technique (GIT)}

The GIT approach to attenuation estimation considers the decay of the spectral amplitude with distance. It simultaneously separates the source, site and attenuation terms by inverting the body wave spectra (e.g. Andrews, 1986; Hartzell, 1992; Castro et al., 1990), thereby yielding information about the characteristics of the source, site amplification and the attenuation of the body waves on their propagation from the source to the receiver. The generalized inversion method is more flexible with respect to calculating $Q$ for body waves than the spectral ratio method because it can be applied to a nonhomogeneous dataset (i.e. one in which not all of the events are recorded by all seismic stations). Consequently, this technique can be applied to spectral amplitudes from both small and bigger events to define the attenuation function describing the $Q$ model for a particular region (Castro et al., 1990). Furthermore, the accuracy of the method improves as more earthquakes are included in the dataset (Field and Jacob, 1995). As the coda wave decay method also does, the general inversion technique yields a frequency-dependent quality factor for body waves. However, the method requires assumptions about a geometrical spreading function and at least one site function or one source must be fixed as a constraint to remove a linear dependency between the source, site and path effects. In this study, we use the GIT method because it yields frequency-dependent $Q$ models and provides additional products in terms of site and source effects. The implementation of the method is described in detail in Chapter 3. 


\subsection{Seismic attenuation in volcanic regions}

Geological properties in volcanic regions are strongly complex and heterogenous. The presence of ash layers and other volcanic materials at the surface and the presence of fluidfilled cracks, magma intrusions and hydrothermal reservoirs in deeper parts of the region will affect the propagation of seismic waves (e.g. Arevalo et al., 2003). This complex composition causes attenuation in volcanic areas to be generally higher than that in other tectonic environments.

Table 2.2 Typical $Q_{0}$ and power law frequency-dependence coefficient $(\alpha)$ for $\mathrm{S}$-wave in different tectonic environments

\begin{tabular}{llll}
\hline \hline Location & Source & $Q_{0}$ & $\alpha$ \\
\hline Volcanic region, southern part of Italy & Castro et al. (2008b) & 17.80 & 1.30 \\
The back-arc, the Aegean sea, Greece & Polatidis et al. (2003) & 55 & 0.91 \\
Subduction zone, Guerrero, Mexico & Castro et al. (1990) & 96 & 0.96 \\
The crust, the southern Kanto, Japan & Kinoshita (1994) & 130 & 0.70 \\
The crust of Garda, Italy & Castro et al. (2008c) & 160 & 0.60 \\
\hline
\end{tabular}

There have been several studies concerning $Q$ estimation in volcanic regions. For instance, Del Pezzo et al. (1985) calculated the direct S-wave quality factor at the Campi Flegrei volcanic region, Italy, using the coda- $Q$ waves decay method applied to shallow earthquakes. They found a frequency-dependent $Q$ that is different from other tectonic regions and inferred that the presence of magma affects the main mechanisms of attenuation, namely anelasticity and scattering. Low $Q$ values $(<40)$ obtained using P-wave dispersion at Campi Flegrei were reported by Villa et al. (1997), suggesting that this region has a very low quality factor. Castro et al. (2008b) measured S-wave attenuation in a highly active volcanic region in the Apennines, in the southern part of Italy, using the spectral amplitude decay with distance method to extract the attenuation function. They found a low quality factor for the lower crust in this region, namely $Q_{S}=17.80 f^{1.30}$. They argue that the high 
attenuation in this area is due to brittle-ductile deformation during past tectonic events.

A recent 3D tomography attenuation model of the Taupo Volcanic Zone, New Zealand (Styles, 2009), reveals very low S-wave $Q$ of $<350$ at $10 \mathrm{~Hz}$ in the forearc crust. Styles concluded that this region is fluid-rich due to the dehydration of sediments carried down with the slab and the presence of hydrous fluids transported in the fractured crust. A combination shear-wave splitting and attenuation at depths of $18 \mathrm{~km}$ and $25 \mathrm{~km}$ in her study shows that the slab-parallel fast orientation in the forearc is located within the lowattenuation region, suggesting that the fractures and cracks extend to at least $25 \mathrm{~km}$ beneath that region. These results also imply a strongly heterogeneous structure of the region as a whole. In order to reveal the main mechanism of attenuation a study based principally on attenuation anisotropy is necessary.

\subsection{Seismic attenuation anisotropy}

As described above, a variety of mechanisms - such as the presence of fluid-filled cracks, internal friction along grain boundaries and scattering due to inhomogeneities-cause attenuation of seismic waves. Anisotropy in seismic attenuation occurs if there is a preferential orientation or direction on the crystals, cracks and heterogeneities. Thus, for the anisotropic case, the attenuation depends upon the wave propagation direction.

Several methods and theories have been developed to investigate the anisotropy of attenuation. Theoretical crack models (Hudson, 1981) predict that the seismic waves that have particle motions perpendicular to fracture strike should be more attenuated than those which are parallel to the crack's orientation. This theory is relatively straightforward to explain intuitively, since the waves propagating parallel to the cracks' orientation will propagate faster and be less attenuated by scattering. Attenuation anisotropy in the near-surface was investigated using the spectral amplitude method by Liu et al. (2005), who measured attenuation anisotropy in the uppermost $200 \mathrm{~m}$ of the crust at the borehole seismometer CHY in Taiwan. The $Q$ values obtained range from 61 to 68 for the fast shear wave and 43 
to 52 for the slow shear wave. They estimated $23-30 \%$ of attenuation anisotropy suggesting the presence of microcrack alignment, which also causes $8 \%$ velocity anisotropy at the same station. Anisotropy of attenuation has also been detected in an active oil-field using a microseismic dataset (Carter and Kendall, 2006). In that case, using the spectral ratio method, an intriguing result was reported in which the waves traveling parallel to the fracture strike as measured by S-wave splitting are sometimes more attenuated than those in perpendicular directions. This result contradicts Hudson's theory and therefore the results require further examination.

Lab-scale measurements have also been used to examine attenuation anisotropy in rock samples containing aligned cracks. Tao and King (1990) measured attenuation from anisotropic rocks and isotropic samples using the spectral ratio method (Section 2.2.1). They observed that attenuation anisotropy is generally accompanied by velocity anisotropy, suggesting that grain boundaries and aligned cracks are responsible for both phenomena. They concluded that attenuation anisotropy can be used as a tool for fracture detection in oil exploration. At a very different scale, the variation of attenuation of the Earth's inner core with direction and geographic location has been quantified by Yu and Wen (2006). The observations suggest that the Earth's inner core is anisotropic with respect to attenuation with high attenuation being related to fast propagation (parallel to velocity anisotropy), opposite to the relations for cracks. For example, they found a $Q$ value of around 200-250 along polar paths and an average $Q$ value of 600 along equatorial paths, which correspond to a polar-equatorial velocity anisotropy where the P-wave in the polar paths travels $1.3 \%-2.8 \%$ faster than in the equatorial paths. They argued that such phenomena may be due to different alignments of the hexagonal close-packed (hcp) iron crystals.

Those measurements imply that attenuation anisotropy contains valuable information to diagnose the structure and properties of the Earth at a broad range scales. However, observations of attenuation anisotropy are sparse compared to those of velocity anisotropy and, to our knowledge, no attempts have been made to quantify attenuation anisotropy in any volcanic regions in the world or any where in New Zealand. As discussed in Sec- 
tion 2.3, volcanic regions commonly have high attenuation due to either intrinsic process or fluid-filled cracks. We expect that if attenuation is caused by intrinsic process and there is indication of attenuation anisotropy, it should exhibit a weak frequency-dependence, while if it is caused by fluids in aligned cracks, there will be a strong frequency-dependence of attenuation (Aki, 1980b; Giampiccolo et al., 2007). 


\section{Chapter 3}

\section{Methodology and data processing}

As discussed in Section 2.2.4, measurements of spectral decay with distance using the generalized inverse technique analyze seismic datasets for source, attenuation and site effects simultaneously. The advantages of using this method are that it yields frequency-dependent $Q$ models for body waves, can be applied to large datasets describing the $Q$ model for a particular region, and provides additional products for further seismic analysis in terms of site and source effects.

\subsection{The generalized inversion technique (GIT)}

A seismic record $d(t, r)$, which has been already corrected for the instrument response, can be described in the time domain by a convolution

$$
d_{i j}(t, R)=s_{i}(t, R) * a(t, R) * z_{j}(t, R)
$$

where $s_{i}$ is the source term, $a$ is the path effect and $z_{j}$ is the site function (e.g. Aki and Richards, 1980). Consequently, in the frequency domain the observed spectrum can be obtained by multiplying the source term $S$, the propagation path $A$ and the site function $Z$ :

$$
D_{i j}(f, R)=S_{i}(f) \cdot A(f, R) \cdot Z_{j}(f)
$$

Here capital letters denote the spectra of the corresponding lower-case variables. Equation 3.2 provides the basic model for the observed seismic spectra and also constitutes the basic 
part of the generalized inversion technique.

Equation 3.2 may be transformed into linear form by taking the logarithm of both sides:

$$
\log D_{i j}(f, R)=\log S_{i}(f)+\log A(f, R)+\log Z_{j}(f)
$$

The linear equation above can be written in matrix form as:

$$
G \bar{m}=\bar{b}
$$

In this equation, $b$ is the data vector of observed spectra, $m$ is a model vector containing the unknown parameters describing the source, attenuation and site terms, and $G$ is the matrix linear operator (see, e.g. Menke, 1989). A problem that commonly arises when using this approach is the linear dependence between the source, attenuation and site terms, so we should make sure that the solution is well constrained for each variable. The least squares solution $\bar{m}=\left(G^{T} G\right)^{-1} G^{T} \bar{b}$ of the predicted model can be computed, for example, by least square inversion (LSQ) or singular value decomposition (Menke, 1989). In general the accuracy of this approach can be improved by increasing the number of earthquakes in the dataset (Field and Jacob, 1995).

The GIT was first used to separate the geometric-attenuation-corrected data into source and site terms by constraining the solution of the model with a known site response at particular stations (Andrews, 1986; Hartzell, 1992). Some stations are chosen as reference sites, in which the site effects are considered flat over the frequency band of interest; for example, one station on hard rock may be constrained to have a flat response of 1 . The reference site also can be determined by setting the average of several stations to 1 . This approach has been developed in order to invert simultaneously for the source, attenuation and site terms. Castro et al. (1990) and Salazar et al. (2007) parameterized attenuation function as a particular function of distance, and another approach is to assume that the attenuation function decays monotonically as a function of distance without specifying a particular shape of the function (the non-parametric method, e.g. Castro et al., 1990, 1996, 2003; Oth et al., 2008, 2009). In this study we only use the GIT-non parametric method, 
because the attenuation function obtained can be corrected with any kind of geometrical spreading form to determine $Q$ without recomputing the inversion (Castro et al., 1990). We discuss this latter approach in more detail below.

\subsubsection{Nonparametric inversion method}

The source, attenuation and site terms are calculated in two spectral inversion steps using the non-parametric method proposed by Castro et al. (1990).

\section{Step 1: Determination of attenuation function and $Q$}

In the first step, the frequency- and distance-dependence of the observed spectra are written as:

$$
\begin{aligned}
D_{i j}\left(f, R_{i j}\right) & =M_{i}(f) \cdot A\left(f, R_{i j}\right) \\
& =M_{i}\left(S_{i}(f), Z_{j}(f)\right) \cdot A\left(f, R_{i j}\right)
\end{aligned}
$$

Here $D_{i j}\left(f, R_{i j}\right)$ is the observed spectrum of earthquake $j$ at station $i, M_{i}(f)$ is a scalar which depends on the size of the earthquake $j$ and also includes source effects $S_{i}(f)$ and the average site effects $Z_{j}(f)$ (Castro et al., 1990, 2008a,b), $R_{i j}$ is the hypocentral distance of event $j$ to site $i$, and $A\left(f, R_{i j}\right)$ is the attenuation function, which incorporates with this formulation both geometrical spreading and anelastic attenuation. The shape of the attenuation function is independent of the size of the event, so that it is possible to combine the spectral amplitude from small and bigger events to define the attenuation function (Castro et al., 1990, 1997).

The attenuation function determined using the non parametric method is based on the fact that the amplitude decay of S-waves with distance is fully governed by geometrical spreading, scattering and anelastic attenuation, which together can be expressed as:

$$
A\left(f, R_{i j}\right)=G\left(R_{i j}\right) \times e^{\frac{-\pi f R_{i j}}{\beta Q_{s}}}
$$

Here $G\left(R_{i j}\right)$ is the geometrical spreading function, $f$ is the frequency, $\beta$ is the shear wave velocity, $Q_{s}$ is the S-wave quality factor and $R_{i j}$ is the hypocentral distance between event 
$j$ and the site $i$. This approach searches for monotonic decay curves and is constrained to be smooth as a function of distance. These requirements mean that the computed spectral amplitudes decrease slowly with distance and that any rapid undulations are considered to be due to site effects and other wave propagation effects. In other words, the residuals at each station between the observation and the model obtained using this method can be interpreted as the site effects (Castro et al., 1990, 2008a,b).

The method assumes that at $R=0$, the spectral amplitude is controlled by the source term $S_{i}(f)$, so that at reference distance $R_{0}, A\left(f, R_{0}\right)$ has a value equal to 1 . By taking the logarithm, equation 3.5 can be made into a linear system of equations

$$
b=m_{i}+a_{j}
$$

in which $b=\log D_{i j}\left(f, R_{i j}\right), m_{i}=\log M_{i}(f)$ and $a_{j}=\log A\left(f, R_{i j}\right)$. In matrix form, this equation can be expressed (Castro et al., 1990; Oth et al., 2008), as follows:

$$
\left[\begin{array}{cc}
\bar{G}_{a} & \bar{G}_{m} \\
\bar{W} & 0
\end{array}\right]\left[\begin{array}{l}
\bar{a} \\
\bar{m}
\end{array}\right]=\left[\begin{array}{l}
\bar{b} \\
0
\end{array}\right]
$$

$G_{a}$ is a matrix representing the parameters related to the attenuation function $(a)$, whereas $G_{m}$ is a matrix related to the model space $m$ containing source and site terms. $W$ is a matrix containing weighting factors $w_{1}$ chosen so that $a_{1}=0$ at $R=0$ (reference distance) and $w_{2}$ chosen to weight the second derivative of the curves and determine the degree of smoothness of the model. The matrix $W$ can be written as:

$$
W=\left[\begin{array}{cccccc}
w_{1} & 0 & 0 & 0 & 0 & \ldots \\
-w_{2} / 2 & w_{2} & w_{2} / 2 & 0 & 0 & \ldots \\
0 & -w_{2} / 2 & w_{2} & w_{2} / 2 & 0 & \ldots \\
. & . & . & . & . & \ldots
\end{array}\right]
$$

The weighting factors are applied to suppress rapid undulation related to site effects and to preserve the monotonic characteristic of the attenuation function. The optimal weighting factors are chosen by visual examination of the model to determine which give sufficiently 
smooth attenuation functions. When choosing the weighting factor $w_{2}$, we should be careful because if it is too high, it will give a straight line which means that the attenuation function cannot preserve properly the variations of attenuation characteristics with distance (Fig. 3.1). If it is too low, the attenuation function still has large variations over very small distance ranges, which means the site effect is not effectively suppressed (Oth, 2007) (Fig. 3.1).

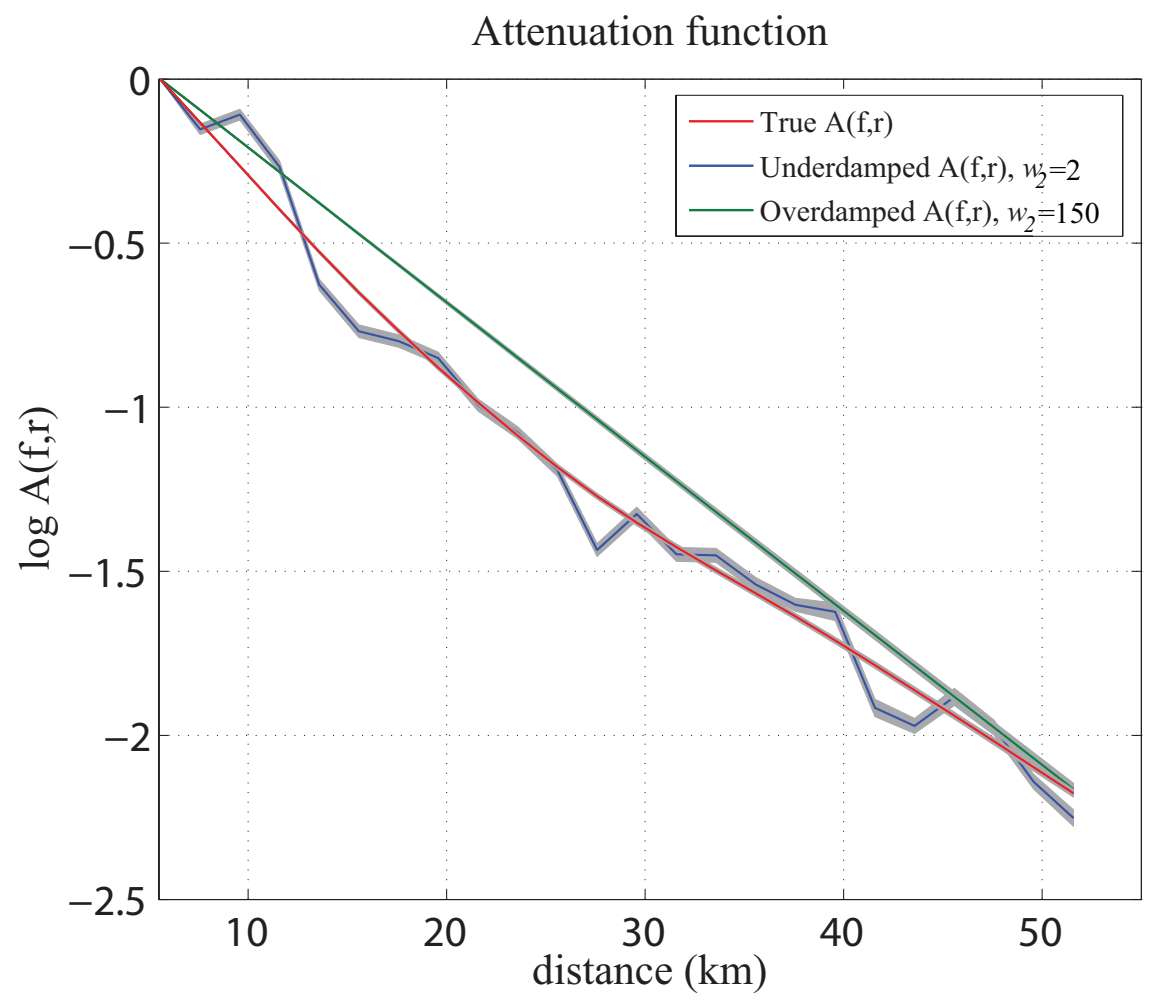

Figure 3.1 The effect of applying weighting factors to the attenuation function.

The discretization of earthquake hypocentral distances for the attenuation function into a number of bins is also important. Here, the bin widths should be equal and contain enough data points to prevent the instability of the attenuation function due to inadequate suppression of undulation (Fig. 3.2). Choosing a small bin width may result in empty bins. In the matrix system, these empty bins will be merged with the previous bin which has data points, causing the bin widths to be unequal. That will notably affect the bins at a greater 
distance as they are determined by the previous unequal bins (Fig. 3.2).

The attenuation function obtained from the non-parametric method has an obvious advantage since it can be examined with any kind of geometrical spreading models without recalculating the previous inversion process. To measure the $Q$ value, we can easily parameterize $A\left(f, R_{i j}\right)$ by taking the logarithm of the equation 3.6 as follows:

$$
\log A\left(f, R_{i j}\right)=\log G\left(R_{i j}\right)-\frac{\pi f \log (e)}{\beta Q_{s}} R_{i j}
$$

The attenuation function can be corrected after defining the geometrical spreading function. Thus, we can rewrite equation 3.10 (after Castro et al., 2003):

$$
y(R)=m R
$$

In which $y(R)=\log A\left(f, R_{i j}\right)-\log G\left(R_{i j}\right)$ and $m=-\frac{\pi f \log (e)}{\beta Q_{s}}$. A linear least squares fit is applied to get the slope $m$ of the corrected attenuation function plotted versus distance. Finally the $Q$ value for a given frequency can be estimated as:

$$
Q(f)=-\frac{\pi f \log (e)}{m \beta}
$$

\section{Step 2: Determination of site and source terms}

In the second step of the inversion process, the corrected spectra from the path effect can be separated into source and site terms for a given frequency $f$ (Castro et al., 1990):

$$
R_{i j}(f)=S_{i}(f) \cdot Z_{j}(f)
$$

where the corrected spectra are described as:

$$
R_{i j}(f)=D_{i j}(f, R) / A(f, R)
$$

Here, $R_{i j}$ represent the spectral amplitudes after correction for the attenuation effects. By taking logarithms, equation 3.13 can be linearized as follows:

$$
\log R_{i j}(f)=\log S_{i}(f)+\log Z_{j}(f)
$$



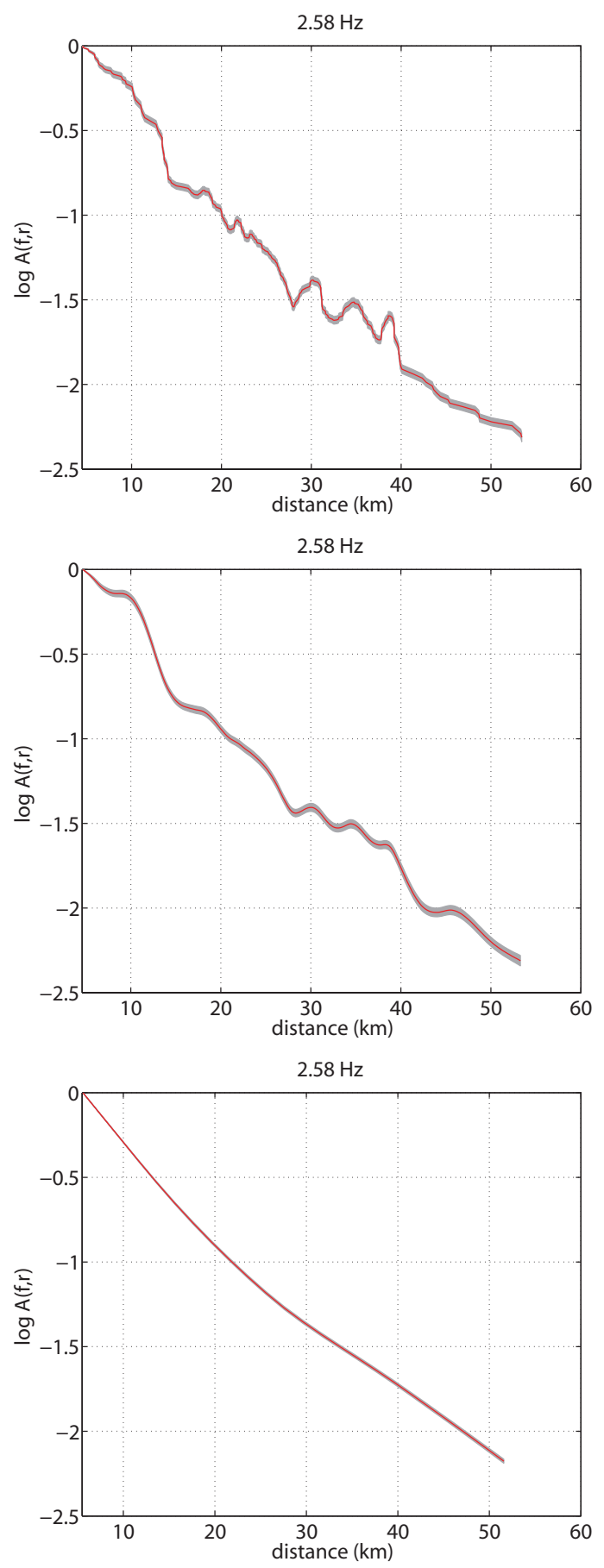

Figure 3.2 An example of attenuation function plots resulting from the inversion of non-parametric method: undiscretized (top panel), discretized using small bin width $0.2 \mathrm{~km}$ (middle) and with an appropriate bin width distance $2 \mathrm{~km}$ (bottom). The solid black line and gray shaded area in the plot represent the mean and the mean \pm one standard deviation of 200 bootstrap replications obtained using bootstrap inversions (e.g. Oth et al., 2008), discussed in more detail later (Section 3.1.2). Note: the standard deviation for the bottom panel is small compared to those for the top and middle panels. 


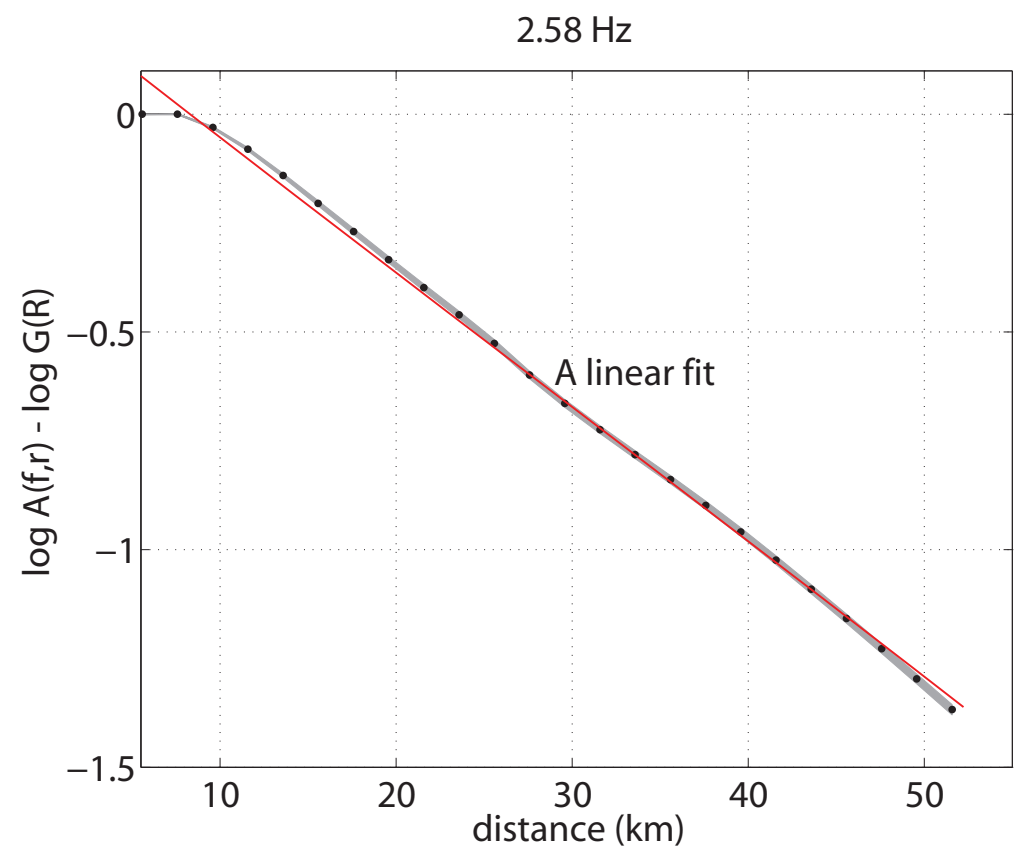

Figure 3.3 An example of a corrected attenuation function from geometrical spreading and a linear square fit to get the slope $m$ value attributed to the $\mathrm{Q}$ value.

and written in matrix form as:

$$
G \bar{m}=\bar{b}
$$

Here $m$ is the model space related to $S_{i}$ and $Z_{j}, G$ is the matrix linear operator and $b$ is the data vector containing the elements $R_{i j}$. Since $G$ is a sparse matrix containing only two non-zero elements in each column or row, this situation requires modification to reduce linear dependence between the site and source terms. One such modification is to constrain at least one station to have a specified site amplification function (a reference site). The reference site is chosen so that the site response is unity at all frequencies (i.e. flat with an amplitude of 1). In this case, equation 3.16 can be rewritten as (Dutta et al., 2001):

$$
\left[\begin{array}{c}
\bar{G} \\
\bar{C}
\end{array}\right] \bar{m}=\left[\begin{array}{l}
\bar{b} \\
0
\end{array}\right]
$$

A row matrix $\bar{C}$ is added in the $\bar{G}$ matrix to constrain the site functions in which $\bar{C} \bar{m}$ is equal to zero. The solution can then be obtained using least square inversion (LSQ) or singular value decomposition. 


\subsubsection{Assessment of the inversion stability}

The accuracy and stability of the inversion results are assessed through bootstrap sample inversion for each analyzed frequency using the same procedure as reported by, for example, Oth et al. (2008). The bootstrap sampling process generates a random sample with replacement from the original data (e.g. Efron, 1979). Some points in the dataset may be selected several times and others not selected at all. For the bootstrap inversion, the technique simply generates a new matrix $G 1$ and a new data vector $d 1$ which have the same size as $G$ and $d$ in equation 3.4. The new matrix is filled randomly with rows from the original dataset and the generalized inverse is computed to get a new solution. This process is repeated for $x$ times to produce $x$ different model solutions (Oth, 2007). For each model solution, we then calculate the mean and the standard deviation of the model parameters. In general, the model solutions of the bootstrap inversion are consistent with the models obtained by inverting the original dataset (Figure 3.4).

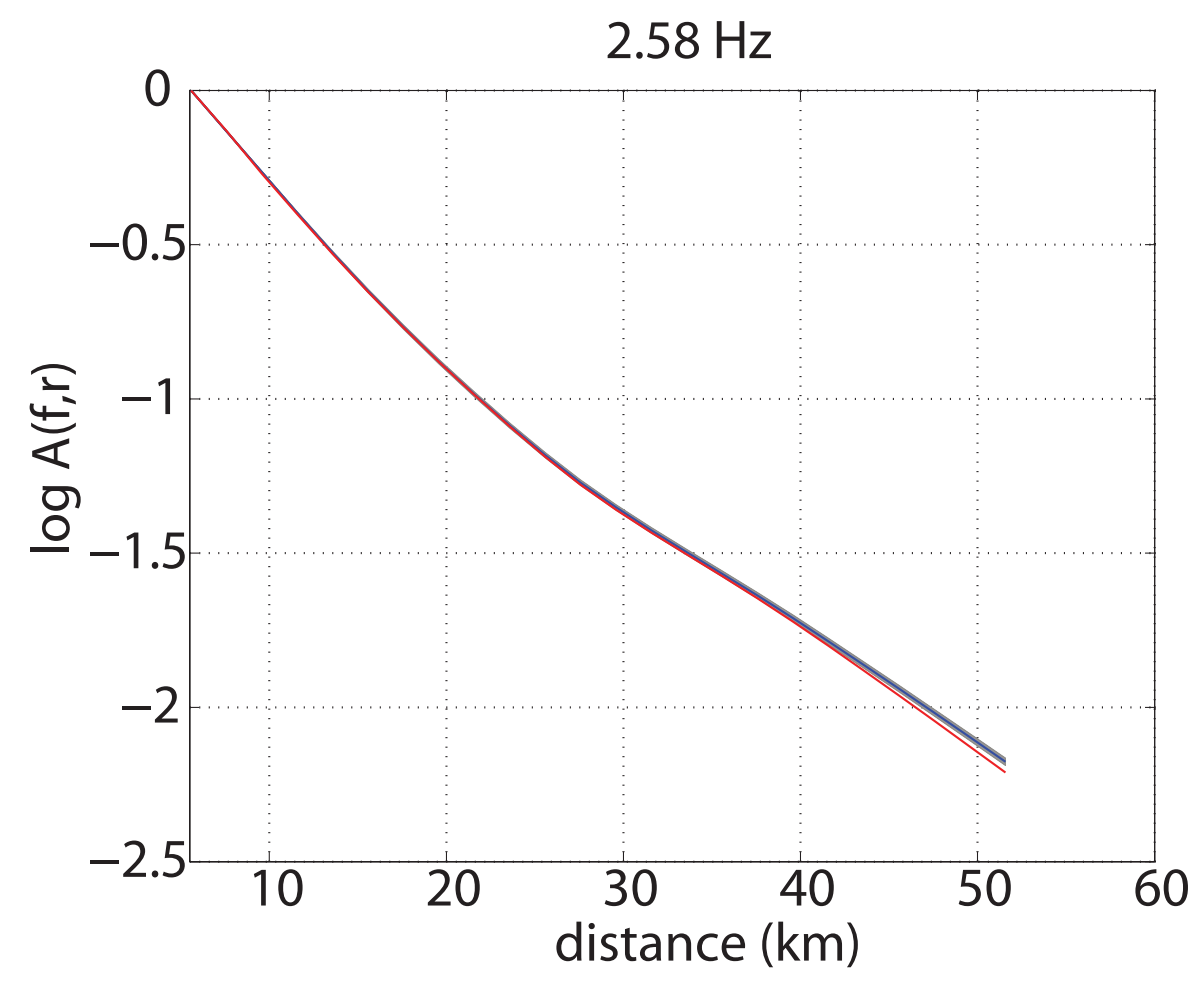

Figure 3.4 Red line: An example of attenuation function obtained by inversion of the original dataset. Blue line and gray shade area: An attenuation function computed by the mean of bootstrap inversion \pm one standard deviation. 


\subsection{Data}

Seismograms of 156 regional shallow earthquakes $(<40 \mathrm{~km})$ recorded at 14 GeoNet seismic stations are analyzed to determine the spectral amplitude decay of S-waves. The 14 stations are situated within $30 \mathrm{~km}$ of the summit of Mt. Ruapehu (Figure 3.5 and Table 3.1), and equipped with short-period and broadband seismographs. The seismograms used in the analysis are all sampled at 100 samples per second with the instrument responses flat between at $1-40 \mathrm{~Hz}$ and $0.08-40 \mathrm{~Hz}$ for the short-period and broadband seismometers, respectively. In this study, only events with magnitudes of $M l \geq 2$ with signal to noise ratio larger than 2 and epicenters within $100 \mathrm{~km}$ of the summit of Mt. Ruapehu are analysed. It is also important to note that only seismograms containing S-phase picked by GNS are used in the dataset, but the picks can be changed manually if needed. This selection may remove some earthquakes containing unpicked S-phase from the dataset.

To examine the effects of attenuation properties with source locations, we divide our dataset into two groups; one containing earthquakes occurring within the TVZ with magnitudes of 2-3.8, whose ray-paths mostly lie south of Lake Taupo (Fig. 3.5 and 3.6), and the other containing earthquakes outside the TVZ with magnitudes of 2-3.2 and ray-paths sampling the southwestern flank of Ruapehu (Fig. 3.5 and 3.8). We then refer to those two groups as the "TVZ" and "non-TVZ" datasets, respectively. The TVZ and non-TVZ datasets contain 90 and 66 earthquakes, respectively (see Appendix A), with more than 315 recordings for each dataset used in the inversion. The hypocentral distances of the earthquakes vary between 5 and $55 \mathrm{~km}$ for the TVZ and, between 5 and $50 \mathrm{~km}$ for the non-TVZ dataset: we discretize the distance ranges of the two cases to 2 and $3 \mathrm{~km}$ binwidth, respectively. Figures 3.7 and 3.9 show vertical cross sections of the selected events for both datasets and indicate that the TVZ events mostly have source depth of 5-10 km, whereas the non-TVZ events mostly occur at depths of 10-20 km. The shallow events for the TVZ data are concentrated at $5 \mathrm{~km}$ depth indicating that these events were probably assigned by convention, because the GeoNet did not determine accurately the focal depths 
of the shallow earthquakes.

Table 3.1 Locations of the GeoNet stations used for analysis

\begin{tabular}{|l|l|l|l|l|}
\hline Name & Code & Latitude $\left(^{\circ}\right)$ & Longitude $\left(^{\circ}\right)$ & Altitude (m) \\
\hline Dome Shelter & DRZ & -39.277 & 175.563 & 2692 \\
Kar West & FWVZ & -39.256 & 175.552 & 2043 \\
Moawhango & MOVZ & -39.408 & 175.752 & 874 \\
Mangateitei & MTVZ & -39.386 & 175.470 & 840 \\
Ngauruhoe & NGZ & -39.177 & 175.600 & 1452 \\
Oturere & OTVZ & -39.164 & 175.664 & 1506 \\
Pokaka & PKVZ & -39.291 & 175.346 & 770 \\
Turoa & TRVZ & -39.300 & 175.547 & 2062 \\
Tukino & TUVZ & -39.269 & 175.653 & 1446 \\
Taurewa & TWVZ & -39.072 & 175.437 & 1084 \\
Wahianoa & WNVZ & -39.328 & 175.597 & 1566 \\
Whakapapa & WPVZ & -39.205 & 175.545 & 1244 \\
West Tongariro & WTVZ & -39.117 & 175.589 & 1186 \\
\hline
\end{tabular}




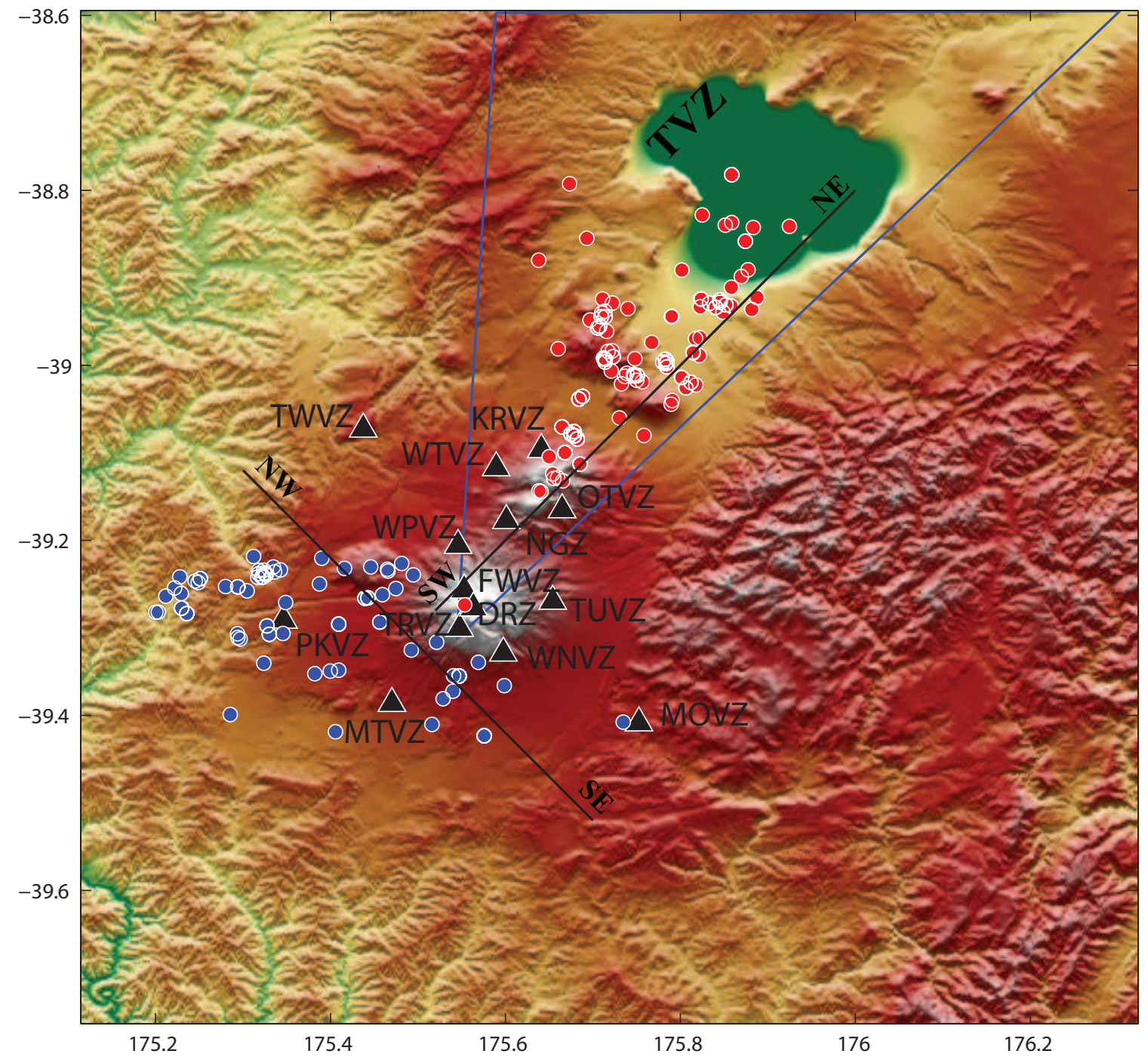

Figure 3.5 The epicenters of the earthquakes used in the analysis are depicted by blue and red circles. The black triangles represent the seismic stations. In total we use 316 records for 'the TVZ dataset (the red circles)' and 338 records for 'the non-TVZ dataset (the blue circles)'. The black lines mark the positions of the cross sections shown in Fig. 3.7 and 3.9. 


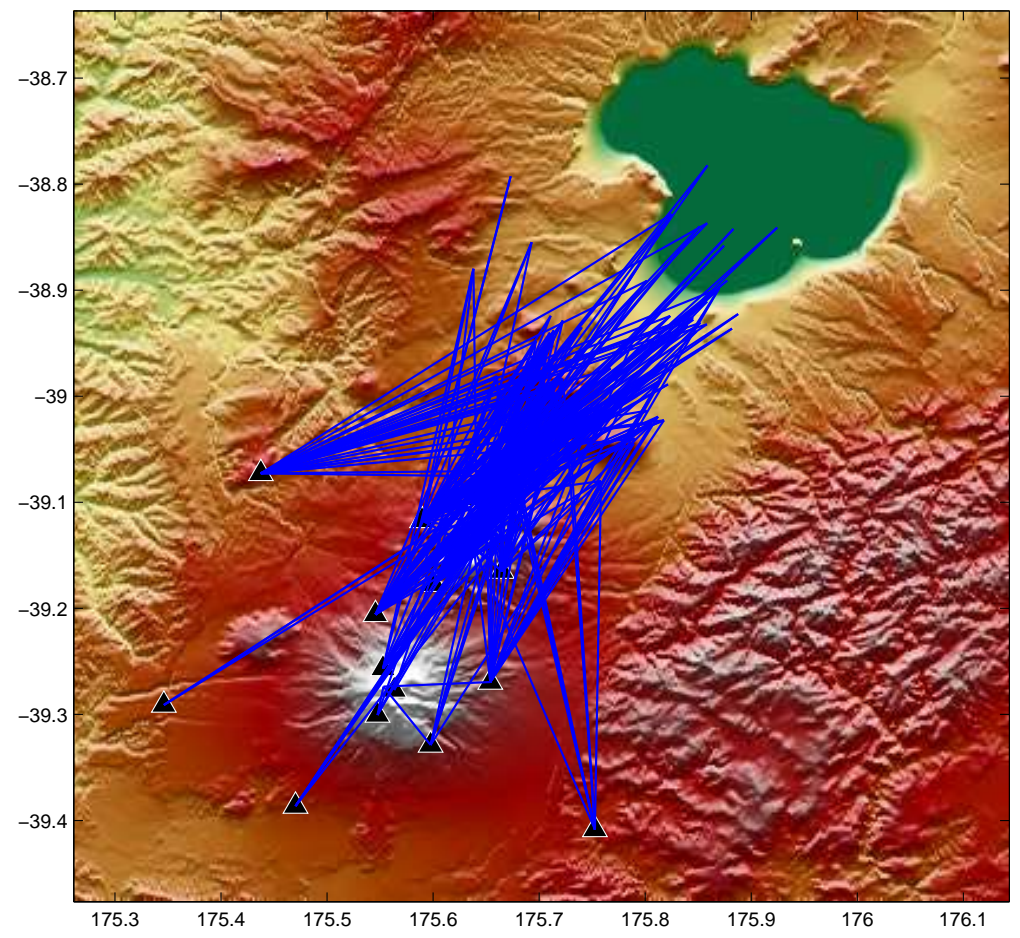

Figure 3.6 Map showing the raypaths of the TVZ earthquakes.

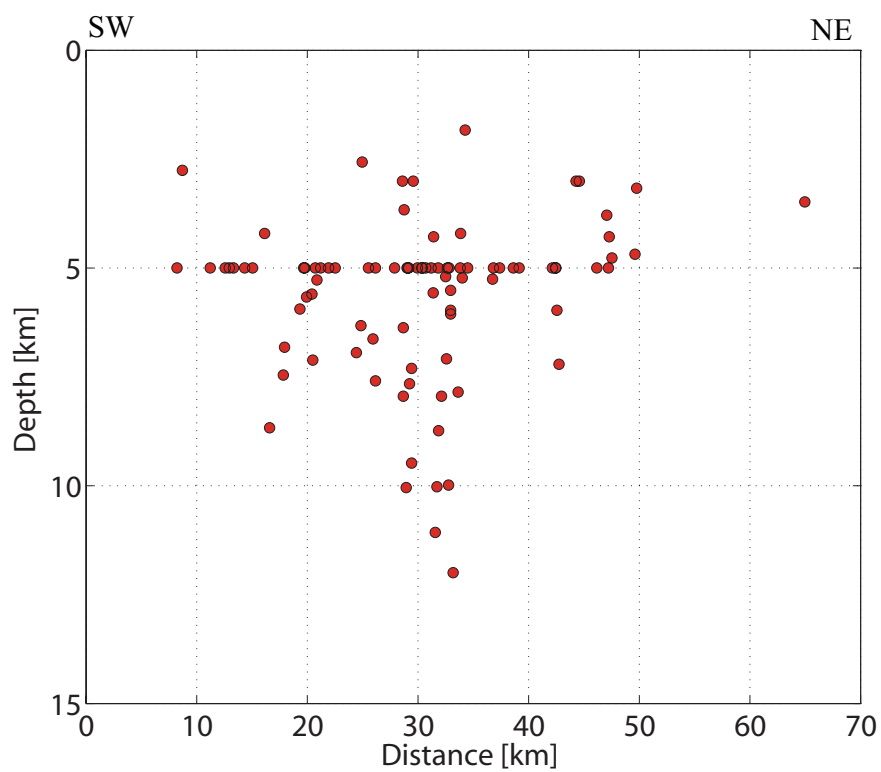

Figure 3.7 A SW-NE vertical cross section of the TVZ earthquakes shown in Fig. 3.5 (black line). 


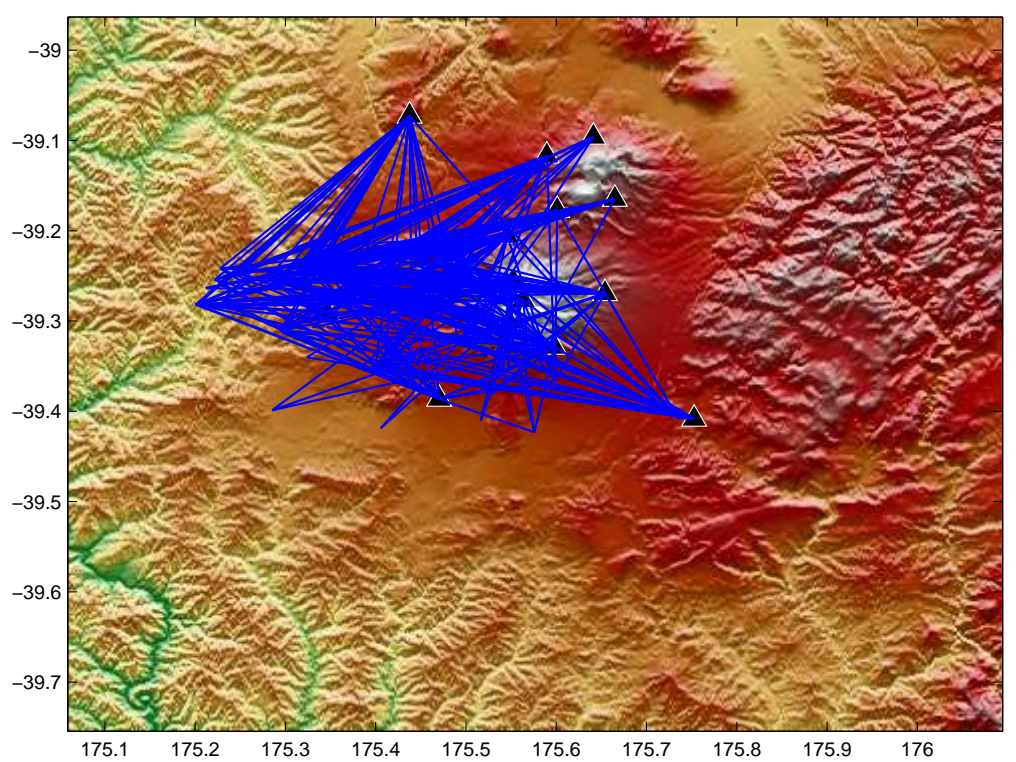

Figure 3.8 Map showing the raypaths of the non TVZ earthquakes.

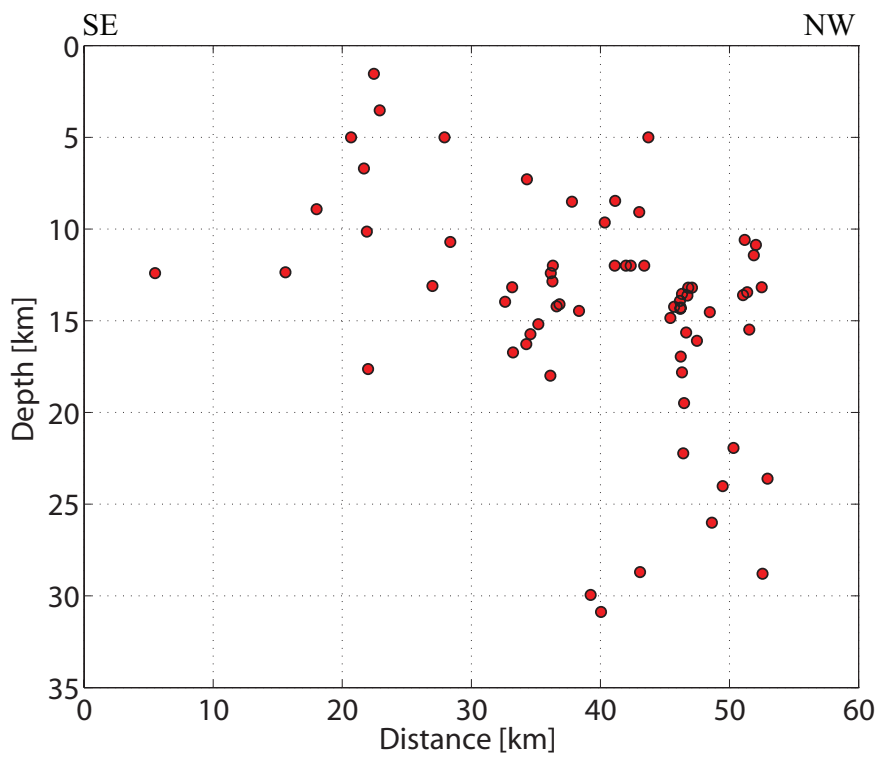

Figure 3.9 A SE-NW vertical cross section of the non TVZ earthquakes on the figure 3.5 (black line). 


\subsubsection{Data preparation}

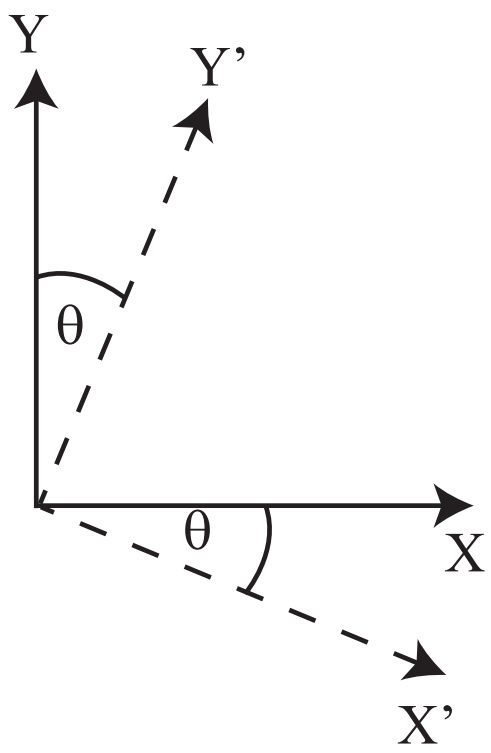

Figure 3.10 The initial pair of horizontal component ( $\mathrm{X}$ and $\mathrm{Y}$ components) which is rotated at an angle of $\theta$

The seismograms used are baseline-corrected by subtracting the mean and removing the instrument response. We rotate pairs of horizontal components (north-south and eastwest components) in steps of $22.5^{\circ}$ from $0^{\circ}$ to $67.5^{\circ}$ and into the radial and transverse directions (Fig. 3.10) to search for the optimal separation of the attenuation curves and thereby determine an anisotropy symmetry system. In order to compute the displacement spectra for each of the rotated seismograms, we select time windows containing clear Swave arrivals. The initial time window starts $1 \mathrm{~s}$ before the $\mathrm{S}$-wave arrival and ends at a time selected automatically that corresponds to the point at which $80 \%$ of the S-wave energy has been achieved. The offset of 1 second has been chosen to assure that the main S-wave arrival energy is included in the analysis. A 5\% cosine taper is applied to each window before the Fourier transform calculation and the signal spectra are smoothed using the Konno-Ohmachi window (Konno and Omachi, 1998):

$$
W_{B}\left(f, f_{c}\right)=\left[\sin \left(\log _{10}\left(f / f_{c}\right)^{b}\right) / \log _{10}\left(f / f_{c}\right)^{b}\right]^{4}
$$

Here $b=20$ is the bandwidth coefficient for smoothing, $f$ and $f_{c}$ are frequency and 


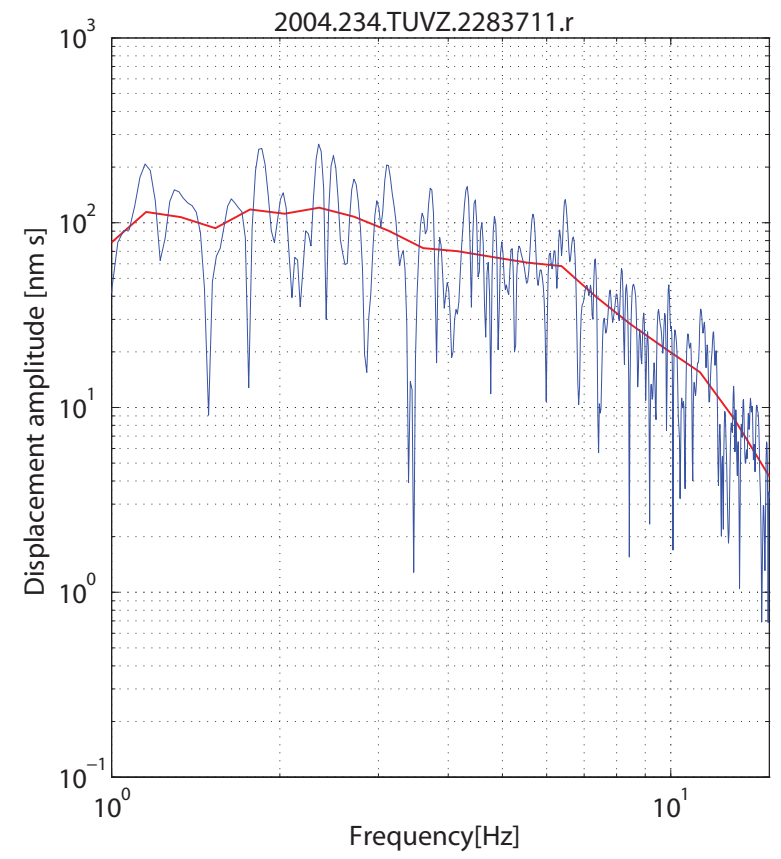

Figure 3.11 Smoothing spectra using the Konno-Ohmachi window

center frequency, respectively. The parameter $b$ controls the smoothing of the spectra: higher values of $b$ will give less smoothing. The advantage of using this function is that the window has a constant width on a logarithmic frequency scale and consequently provides equidistant points in logarithmic scale. In this processing, we compute the smoothed spectra at around 20 frequency points in the range $2-10 \mathrm{~Hz}$ for the TVZ data, and from 2-12 Hz for the non-TVZ data. Noise spectra are calculated using P-coda windows which have the same length as the signal windows. It is important to note that we use P-coda windows as noise windows because some records in our dataset have very small pre-event noise windows, that preclude us from using pre-event (i.e. pre-P) noise as a noise window. The amplitudes of the signal spectra over each frequency band are then compared with the calculated P-coda spectra and only data with signal to "noise" ratios larger than 2 $(S / N>2)$ are retained for the inversion. Figure 3.12 displays some data examples. The left panel shows selected records of horizontal components with their S-wave windows, whereas the right frame corresponds to the displacement spectra using the indicated windows. 

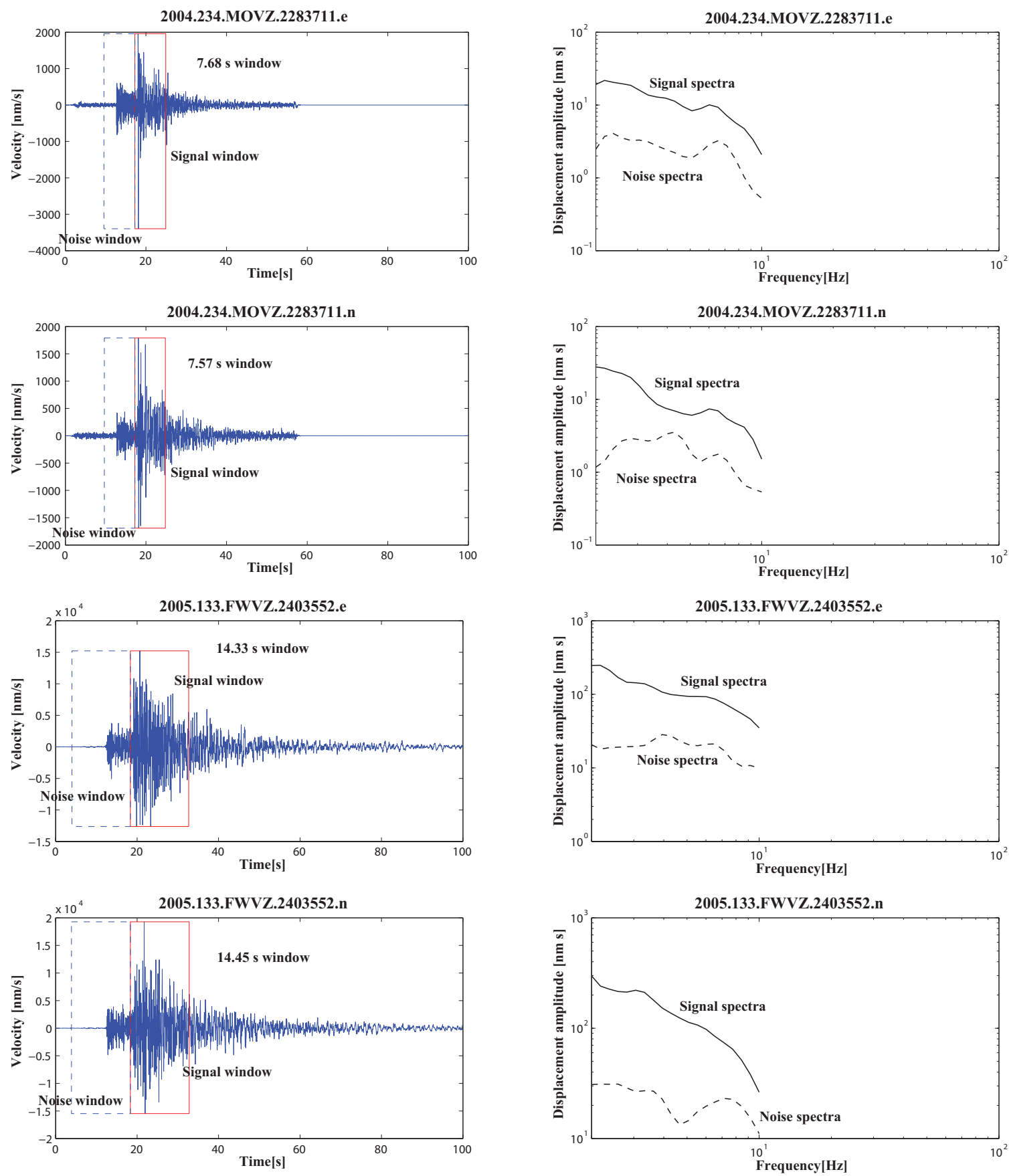

Figure 3.12 Left panel: Example records of the E-W and N-S components; signal and noise windows denoted by the red and blue dashed boxes respectively, used to compute the displacement spectra. Right panel: Examples of the smoothed displacement spectra obtained from the selected windows of the seismogram on the left panel. 


\section{3 $Q$ model}

The attenuation functions obtained by the procedure described in the previous section are used to estimate the $Q$ model. We address the $Q$ estimation process based on two model assumptions: 1. a homogeneous $Q$ model calculated from the attenuation functions assuming a single homogenous layer; 2. a non-homogenous $Q$ model that distinguishes attenuation distance curves of the upper crust from those of the lower crust. It is important to note that the presence of attenuation anisotropy is determined by comparing attenuation models of two rotated orthogonal components. Then, the direction of anisotropy is determined based on the model of two orthogonal components producing maximum separation of the attenuation values.

\subsubsection{Homogenous $Q$ model}

To calculate a homogenous $Q$ model, we examine the amplitude decay function within the entire distance range rewritten as:

$$
A(f, R)=G\left(R_{i j}\right) \times e^{\frac{-\pi f R}{\beta Q_{s}}}
$$

Here, we use an average $S$-wave velocity of $\beta=3.5 \mathrm{~km} / \mathrm{s}$, based on the $S$-wave velocity model beneath the TVZ as obtained by Bannister et al. (2004). We define our geometrical spreading functions following Castro et al. (2008b):

$$
G(R)= \begin{cases}R_{0} / R, & R_{0}<R<R^{\prime} \\ R_{0} /\left(R^{\prime} R\right)^{1 / 2}, & R \geq R^{\prime}\end{cases}
$$

We use this geometrical form to minimize the flattening effect on the amplitude decay function of the secondary arrivals of reflected waves and a change in geometrical spreading function (Castro et al., 1996). Here, $R$ is the hypocentral distance and $\mathrm{R}^{\prime}$ is the hypocentral distance at which the flattening effect on the amplitude decay function begins. In this study, we use $\mathrm{R}^{\prime}=25 \mathrm{~km}$ (see Chapter 4 ). $R_{0}$ is the reference distance, here chosen to be $R_{0}=5$ 
$\mathrm{km}$; we normalize our geometrical spreading function at this distance because most records have hypocentral distances larger than $5 \mathrm{~km}$. Finally, $Q$ is estimated for each frequency using equations 3.11 and 3.12. To describe the frequency dependence of the $Q$ model, we use a model of the form (Aki, 1980a):

$$
Q(f)=Q_{0}\left[\frac{f}{f_{0}}\right]^{\alpha}
$$

where $Q_{0}$ is the value of $Q_{S}$ at $1 \mathrm{~Hz}$ and $\alpha$ is the frequency-dependency coefficient.

\subsubsection{Two-layer $Q$ model}

To investigate the contribution of the crust to the overall attenuation anisotropy, we propose a non-homogenous $Q$ model to separate the effect of attenuation in the upper crust and the lower crust in this region. We expect that the attenuation anisotropy in the upper crust is due to a high density of cracks, fractures and pore space, while the presence of connected melt beneath the TVZ may contribute to the attenuation anisotropy in the lower crust.

Following the same procedure for a two-layer attenuation model as outlined by Castro et al. (2008b), we divide our $Q$ estimation based on the changes in slope of the amplitude decay at different distances. The notation $R^{\prime}$ used below is the same as that used in equation 3.20 .

\section{The upper crust $Q$ model $\left(R<R^{\prime}\right)$}

In this model we modify equation 3.6 to quantify the upper crust $Q$ model as:

$$
A_{u}(f, R)=G(R) \times e^{\frac{-\pi f R}{\beta_{1} Q_{s 1}}} \times e^{-\pi f K}
$$

where $G(R)$ is geometrical spreading function, $\beta_{1}$ is the $\mathrm{S}$-wave velocity in the upper crust (3.2 km/s based on the S-wave velocity model beneath the TVZ; Bannister et al. (2004); Rowlands et al. (2005)), $Q_{s 1}$ is the quality factor in the upper crust and the final term $K$ is the near-surface attenuation (Anderson and Hough, 1984). The inclusion of $K$ enables us to investigate the effects of local geology on attenuation in the upper crust. To estimate 
$Q$ and $K$, we correct the attenuation function for the effects of geometrical spreading. We estimate the geometrical spreading using equation 3.20. Then, we linearize the equation regarding the corrected attenuation function to estimate $Q$ and $K$. Thus, equation 3.22 can be written (Castro et al., 2008a):

$$
y(R)=m R+C
$$

where $y(R)=\log A\left(f, R_{i j}\right)-\log G(R)$ and $m=-\frac{\pi f \log (e)}{\beta Q_{s 1}}$ and $C=-\pi f K \log (e)$.

\section{The lower crust $Q$ model $\left(R>R^{\prime}\right)$}

The lower crust attenuation can be estimated by assuming a model of the form (Castro et al., 2008b):

$$
A_{l}(f, R)=G(R) \times e^{\frac{-\pi f R}{\beta_{2} Q_{s 2}}}
$$

Here $\beta_{2}$ is the $S$-wave velocity in the lower crust $(3.7 \mathrm{~km} / \mathrm{s}$ based on the $S$-wave velocity model beneath the TVZ (Bannister et al., 2004; Rowlands et al., 2005), $Q_{s 2}$ is the quality factor in the lower crust, and $A_{l}(f, R)$ is the corrected attenuation function after the effects of upper crust attenuation have been removed. We correct the attenuation function for the effect of upper crust attenuation using equation 3.22 and the $Q$ and $K$ values obtained from equation 3.23. Thus, the corrected attenuation function for the lower crust can be expressed as:

$$
A_{l}(f, R)=A_{2}(f, R) / A_{1}(f, r)
$$

where $A_{2}(f, R)$ is the observed attenuation function for $R^{\prime}<R$ and $A_{1}(f, r)$ is the attenuation function at $r=R^{\prime}$, the maximum distance of the upper crust in this observation. The geometrical spreading correction is represented using equation 3.20. 


\section{Chapter 4}

\section{Results}

In this chapter, we present attenuation anisotropy results for areas inside and outside the Taupo Volcanic Zone. In Section 4.1, we first display the amplitude decay functions found using the non-parametric GIT method described in Section 3.1. The attenuation anisotropy results for both regions are presented in Sections 4.2 and 4.3. Finally, additional results pertaining to source and directional site effects obtained from the second step of the nonparametric GIT method are shown in Section 4.4.

\subsection{Non-parametric attenuation function inside the TVZ}

S waves consist of two components: S waves' horizontal component $(\mathrm{SH})$ polarized in the horizontal plane parallel to the Earth's surface and S waves' vertical component (SV) polarized in the vertical plane. SH and SV correspond to the transverse and radial directions in term of the direction linking the source and the receiver, respectively. Since seismograms are usually recorded on vertical, east-west $(E)$ and north-south $(\mathrm{N})$ sensors, which rarely correspond to radial $(\mathrm{R})$ and transverse $(\mathrm{T})$ polarization directions, we must rotate the seismograms to determine R-T components in term of the S-wave propagation's direction.

Radial and transverse directions are the natural coordinates used in a layered isotropic 
earth because they separate the components: $\mathrm{P}$ and $\mathrm{SV}$ waves are present on radial components while $\mathrm{SH}$ waves are on transverse component. Examining transverse component allow us to see the effects of horizontally polarized waves, providing information about radial anisotropy, while azimuthal anisotropy is measured between horizontal components.

\section{The effect of rotation}

We perform the non-parametric GIT approach described in Section 3.1 to obtain attenuation functions for two different regions in the southernmost part of the TVZ. The inversion method is applied to the directional spectra after rotating the E-W and $\mathrm{N}-\mathrm{S}$ components in steps of $22.5^{\circ}$ from $0^{\circ}$ to $67.5^{\circ}$ and into radial and transverse components using earthquake dataset described in Section 3.2.1. The purpose of the rotation is to search for the orthogonal direction producing maximum separation of the attenuation functions and thereby identifying an anisotropy symmetry system.

In Figure 4.1, we compare the attenuation functions obtained for the E-W component S-wave (blue lines) and with those for the $\mathrm{N}-\mathrm{S}$ component (dashed red lines) at six frequencies. A similar pattern also can be seen for other directions of seismograms (see Appendix B.1). At low frequencies (2.58 to $4.67 \mathrm{~Hz}$ ), the functions decrease monotonically with hypocentral distance, but a flattening effect starts to appear at around $6.02 \mathrm{~Hz}$, particularly for hypocentral distances greater than $25 \mathrm{~km}$. The flattening effect becomes stronger with increasing frequency (Fig. 4.2). In addition, the decay rates of both components are generally about the same at low frequencies, while at high frequencies the decay of the E component with distance is markedly more rapid than for the $\mathrm{N}$ component.

Figure 4.3 shows the effects of rotating seismograms on the amplitude decay curves at a frequency of $8.44 \mathrm{~Hz}$. In general, for pairs of the orthogonal components, the decay curves of the component lying close to the E-W direction decay faster than those lying away from that direction. For example, the decay curve obtained for the $67.5^{\circ}$ direction attenuates more than that obtained for the orthogonal direction. At rotation angle $\theta=22.5^{\circ}$, there is no significant differences between two components' spectral decay, while the maximum 
difference of two orthogonal components' amplitude decay occurs at the original components $(\mathrm{N}-\mathrm{S}$ vs $\mathrm{E}-\mathrm{N})$ of the seismogram, particularly for hypocentral distances greater than $25 \mathrm{~km}$.

Figure 4.4 shows the amplitude decay functions obtained from the inversion of the spectra calculated for radial and transverse components (i.e. parallel and orthogonal to the ray propagation direction). In general, the results show a striking similarity with the general trend from the E-N amplitude decay model. In the frequency range $2.58-6.02 \mathrm{~Hz}$, and particularly for distance less than $25 \mathrm{~km}$, both amplitude decay curves show the same rate of decay, whereas at high frequencies and hypocentral distance greater than $25 \mathrm{~km}$ the functions obtained from the transverse component (dashed red lines) attenuate more rapidly than those obtained from the radial component. The flattening effect is also observed at high frequencies of the decay function, and is most flat on the radial component. 

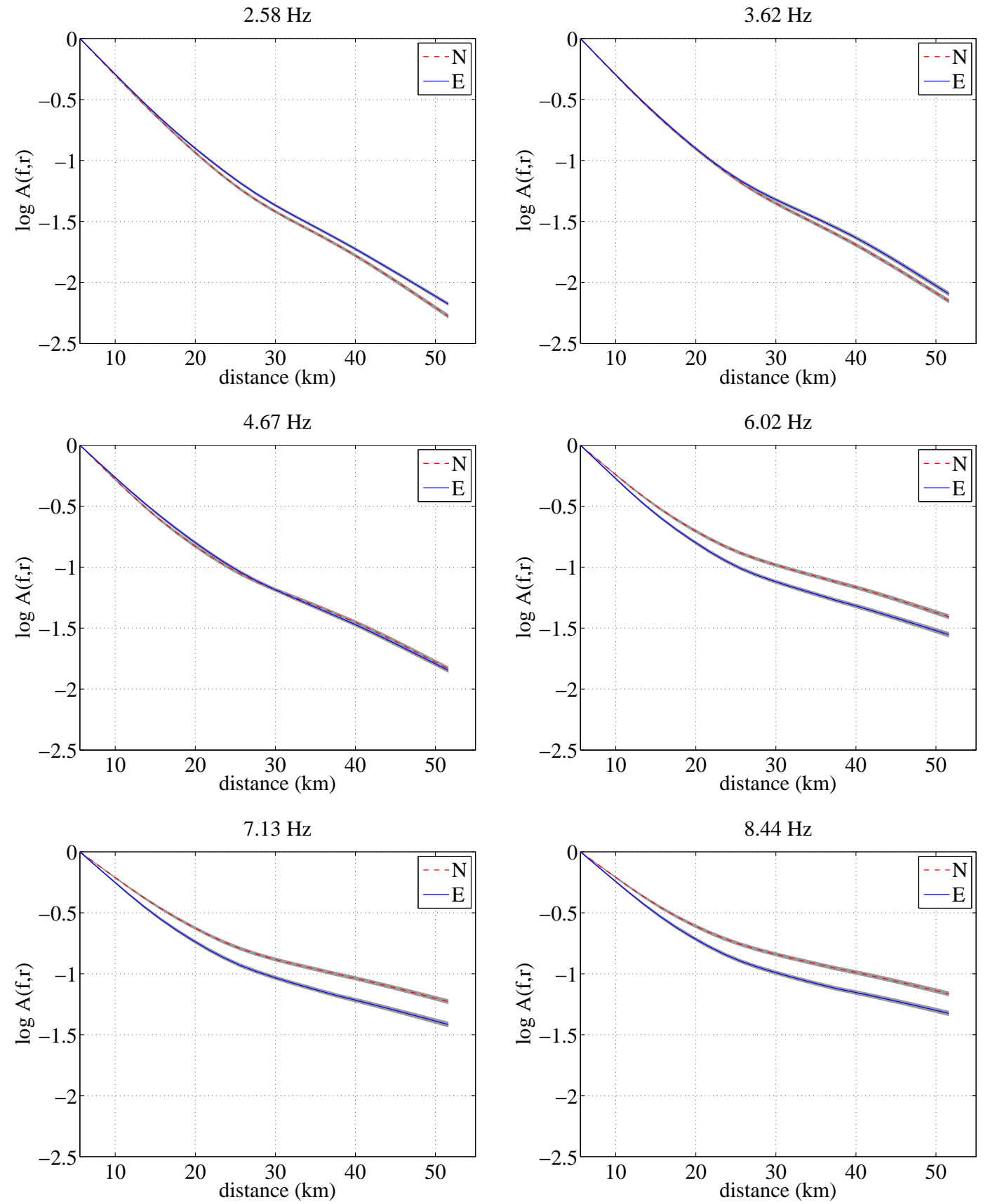

Figure 4.1 Amplitude decay functions versus hypocentral distance for E-W and $\mathrm{N}-\mathrm{S}$ components at six frequencies. The dashed red and blue lines represent the mean of 200 bootstrap samples and the gray shaded area represents the mean \pm $2 \sigma$. 

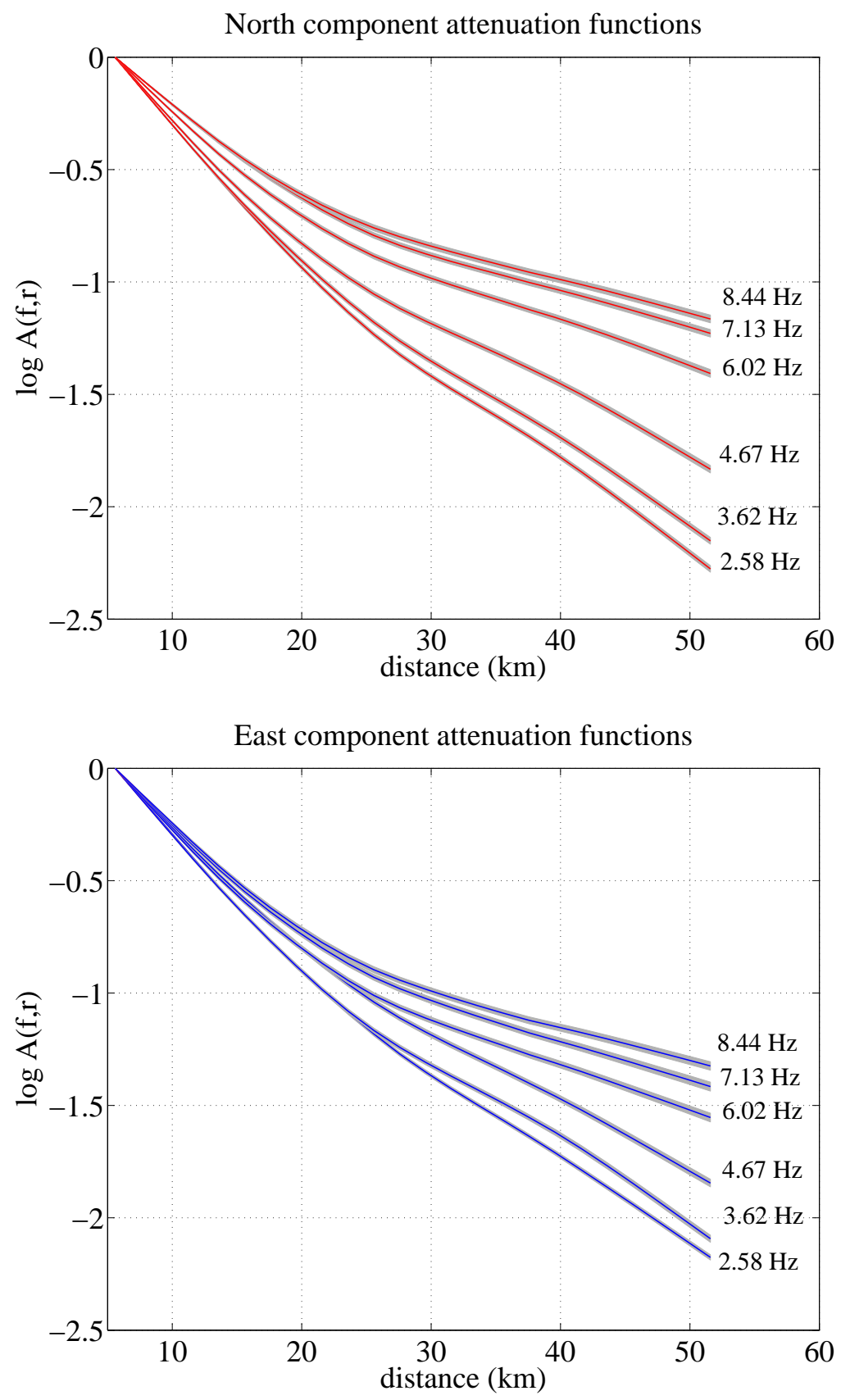

Figure 4.2 Spectral amplitude decay curves with distances obtained using the non-parametric GIT method at six selected frequencies. The top and bottom panels represent attenuation functions for north and east components, respectively. For both components, the spectral amplitude decays faster at low frequencies than at high frequencies. The flattening effect also gets stronger with increasing frequency. 

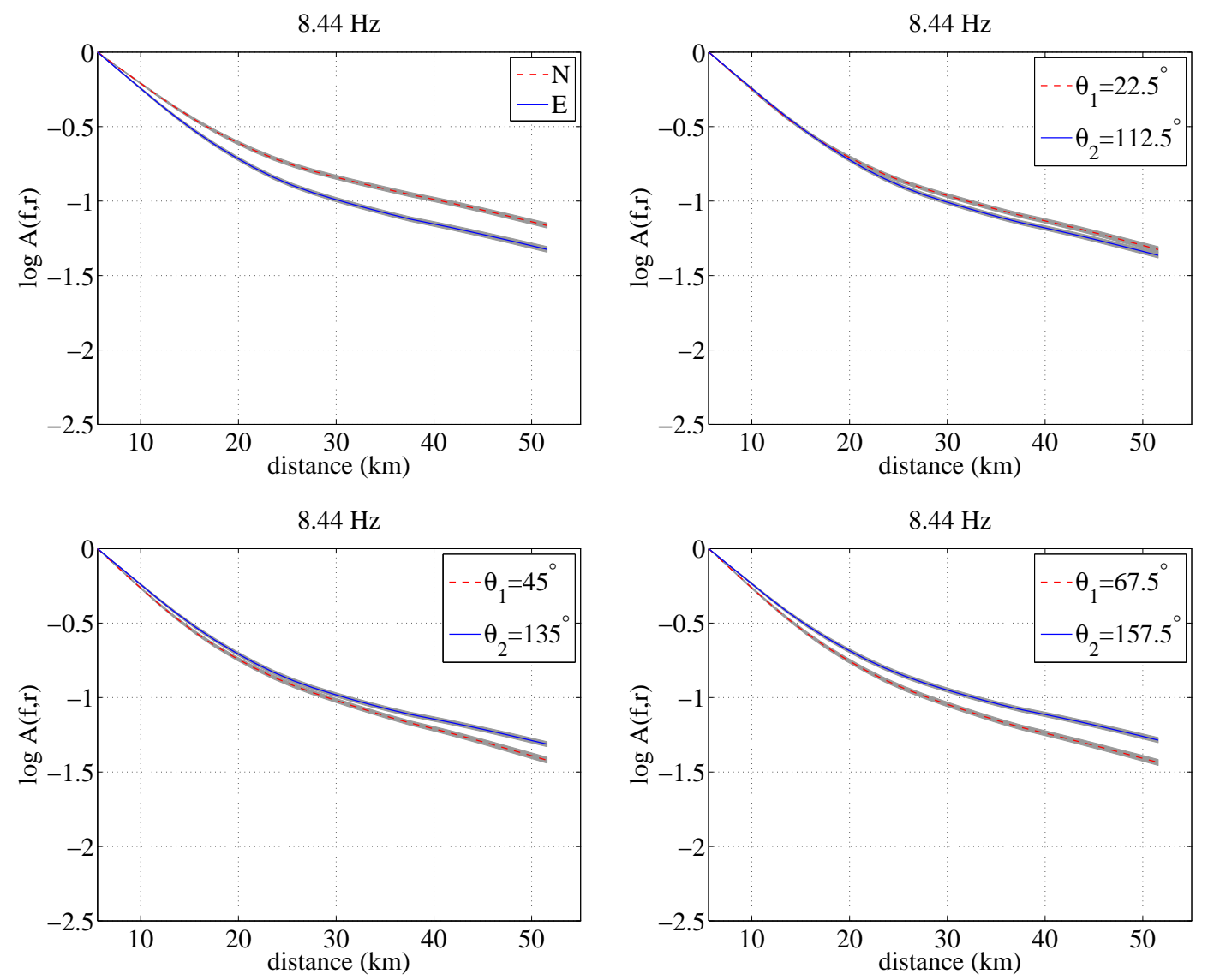

Figure 4.3 Directional effect of amplitude decay curves plotted as a function of hypocentral distance. The dashed red and blue lines represent the mean of 200 bootstrap samples and the gray shaded area represents the mean $\pm 2 \sigma$ 

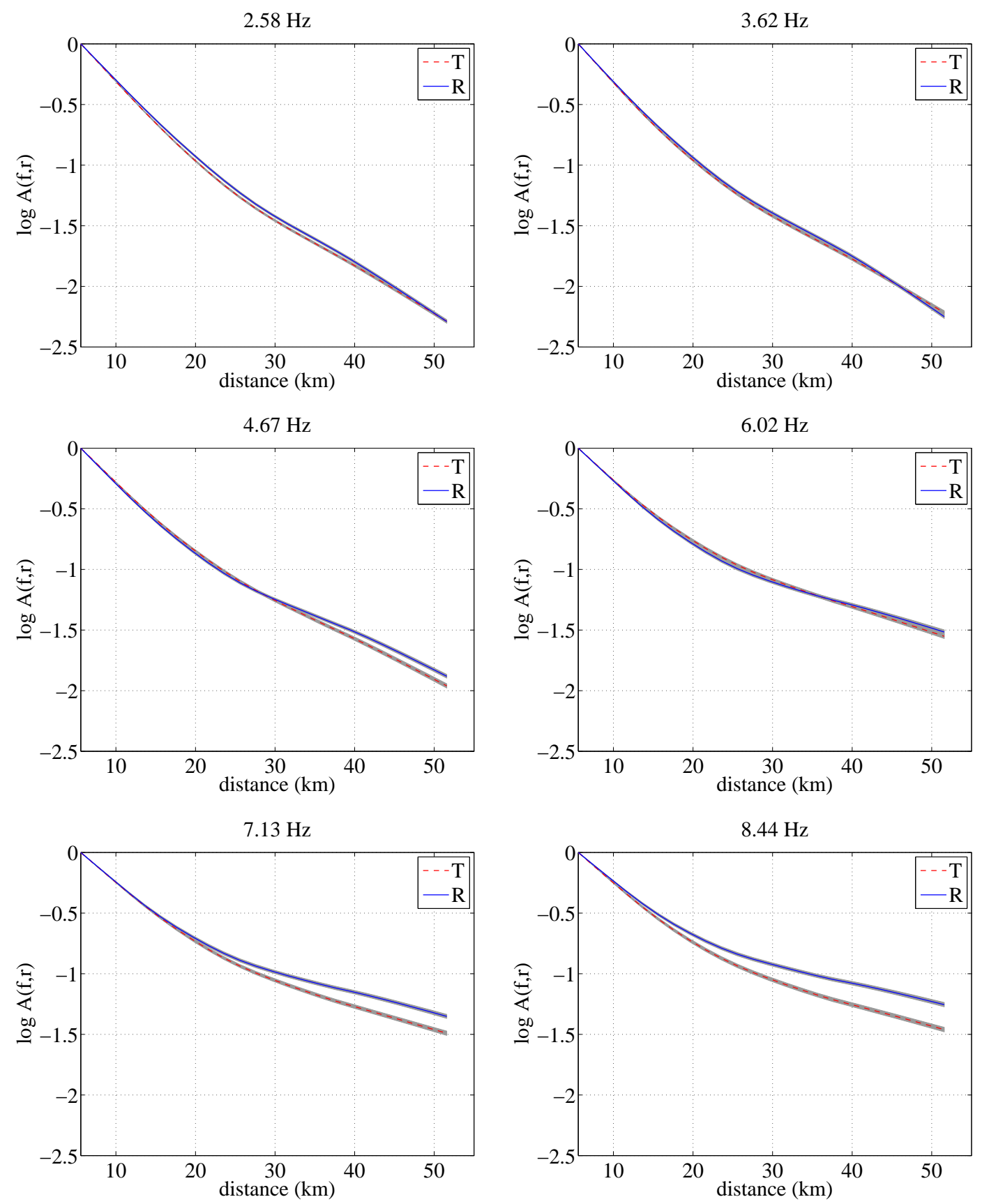

Figure 4.4 Amplitude decay functions versus hypocentral distance for radial and transverse components at six selected frequencies. The dashed red and blue lines represent the mean of 200 bootstrap samples and the gray shaded area represents the mean $\pm 2 \sigma$. The trend of the function are generally the same as observed for $\mathrm{E}$ and $\mathrm{N}$ components. The flattening effect also appear at high frequencies of the decay function, particularly at hypocentral distances $>25 \mathrm{~km}$. 


\section{2 $Q$ model inside the TVZ}

\subsubsection{Homogeneous $Q$ model}

Information about radial and transverse directions is required to determine the distribution direction of both components regarding the S-wave propagation's direction. The rose diagrams in Figure 4.5 show the distribution of directions of the radial and transverse components for the TVZ data. The most common direction of the radial component lies at approximately $215^{\circ}$, whereas the transverse component's directions mostly lie around $305^{\circ}$. The pattern of the rose diagrams also suggests that there could be a dependence between the propagation direction and the polarization azimuth. For example, average radial direction is $210^{\circ}$, which suggests that the decay of $30^{\circ}$ or $120^{\circ}$ may be similar to that of radial and transverse components.
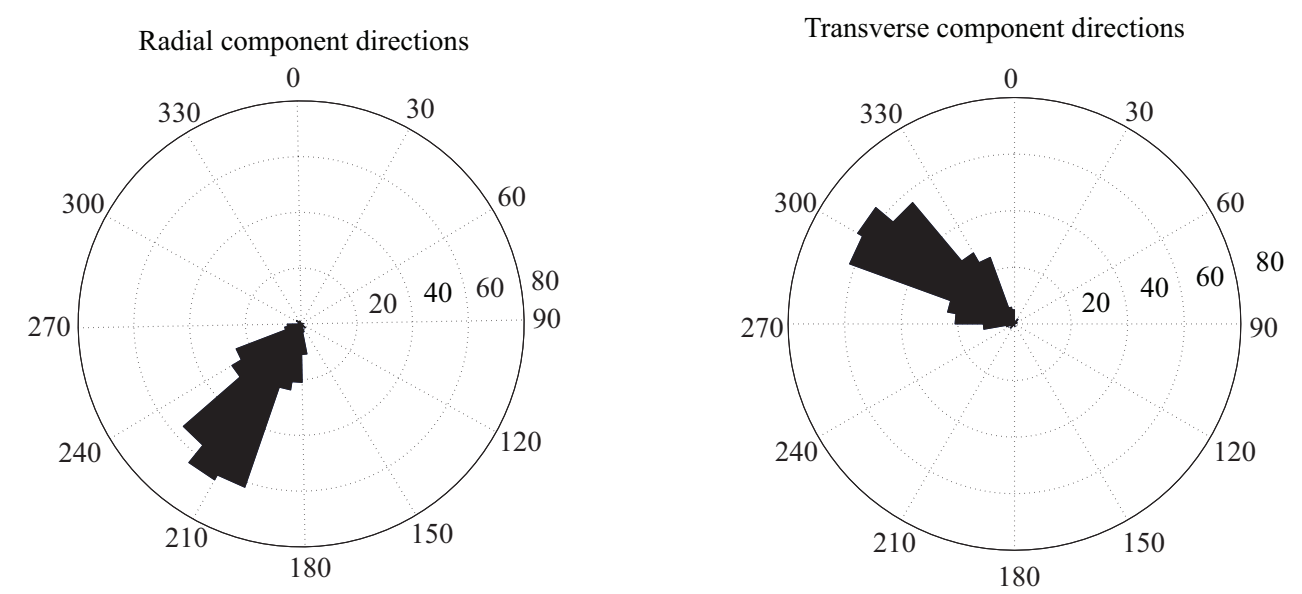

Figure 4.5 Rose diagrams showing the distribution of the radial and transverse components' directions obtained from the TVZ dataset.

We plot the estimated $Q$ for S-waves measured for 20 individual frequencies between 2 and $10 \mathrm{~Hz}$ from the E-W, N-S and all rotated seismograms in Fig. 4.6. At low frequencies $(f<6 \mathrm{~Hz})$, the values $Q$ of the two orthogonal components are generally about the same, with slightly different values of $Q$ at the $2-3 \mathrm{~Hz}$ band for some orthogonal components. At frequencies higher than $6 \mathrm{~Hz}$ only the model of $\mathrm{E}-\mathrm{N}$ and $\mathrm{R}-\mathrm{T}$ have significantly different values of $Q$, suggesting that the shear wave is anisotropic to high frequency propagation 
only. The weak attenuation anisotropy in the $\mathrm{E}-\mathrm{W} / \mathrm{N}-\mathrm{S}$ direction can be approximated with the relation $Q_{S_{E}}(f)=(6.15 \pm 1.22) f^{(1.73 \pm 0.12)}$ and $Q_{S_{N}}(f)=(4.14 \pm 1.26) f^{(2.06 \pm 0.14)}$. Here, the power law function of $Q$ may correspond to the contribution of random scattering or to rheology (e.g. Aki, 1980b) as discussed further in Chapter 5. We summarize the $Q$ values obtained for homogeneous model in Table 4.1.

Table 4.1 Summary of homogeneous $Q$ model $\left(Q=Q_{0} f^{\alpha}\right)$ with $2 \sigma$ confidence level for the TVZ data

\begin{tabular}{ccccc}
\hline Orthogonal components & $Q_{0_{1}}$ & $\alpha_{1}$ & $Q_{0_{2}}$ & $\alpha_{2}$ \\
\hline N-E & $4.14 \pm 1.26$ & $2.06 \pm 0.14$ & $6.15 \pm 1.22$ & $1.73 \pm 0.12$ \\
NNE-ESE & $4.69 \pm 1.23$ & $1.89 \pm 0.13$ & $6.24 \pm 1.19$ & $1.70 \pm 0.11$ \\
NE-SE & $5.29 \pm 1.27$ & $1.76 \pm 0.15$ & $5.48 \pm 1.19$ & $1.79 \pm 0.11$ \\
ENE-SSE & $5.93 \pm 1.25$ & $1.69 \pm 0.14$ & $4.64 \pm 1.21$ & $1.90 \pm 0.12$ \\
R-T & $4.27 \pm 1.26$ & $1.98 \pm 0.14$ & $6.46 \pm 1.19$ & $1.63 \pm 0.11$ \\
\hline
\end{tabular}




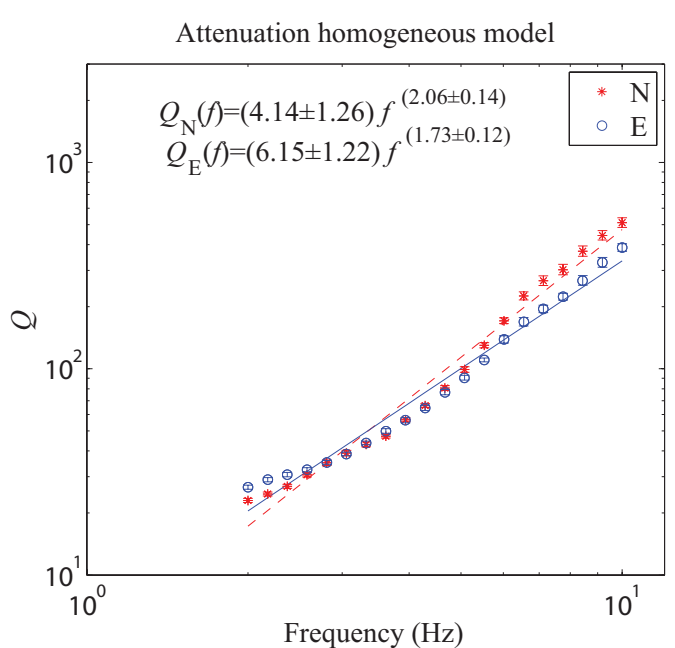

Attenuation homogeneous model

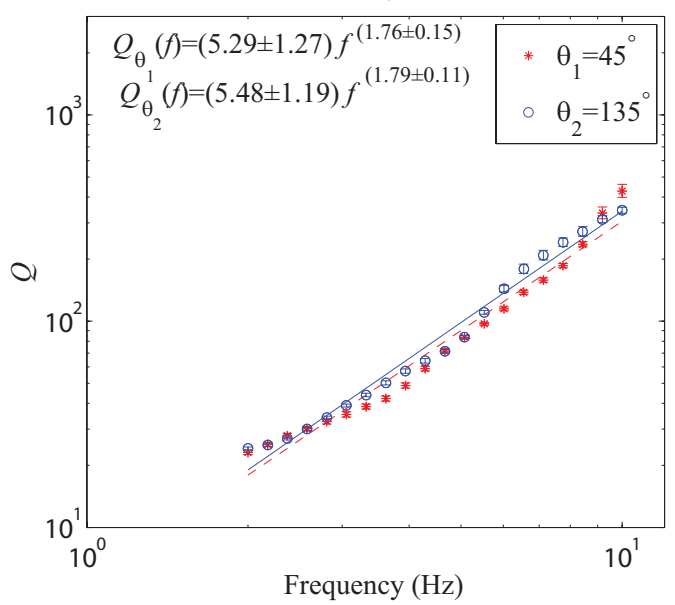

Attenuation homogeneous model

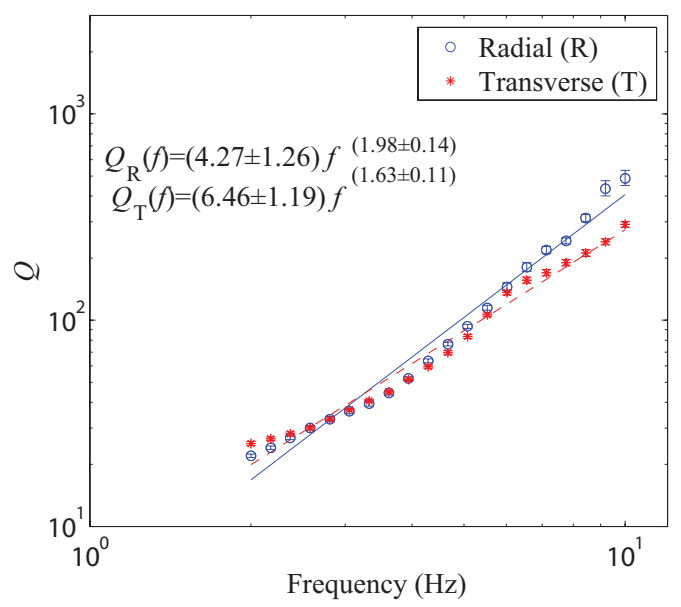

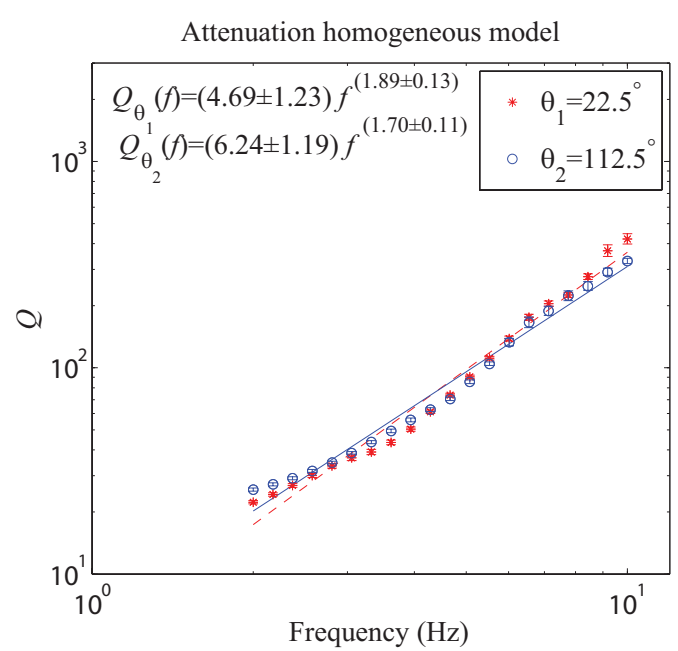

Attenuation-homogeneous model

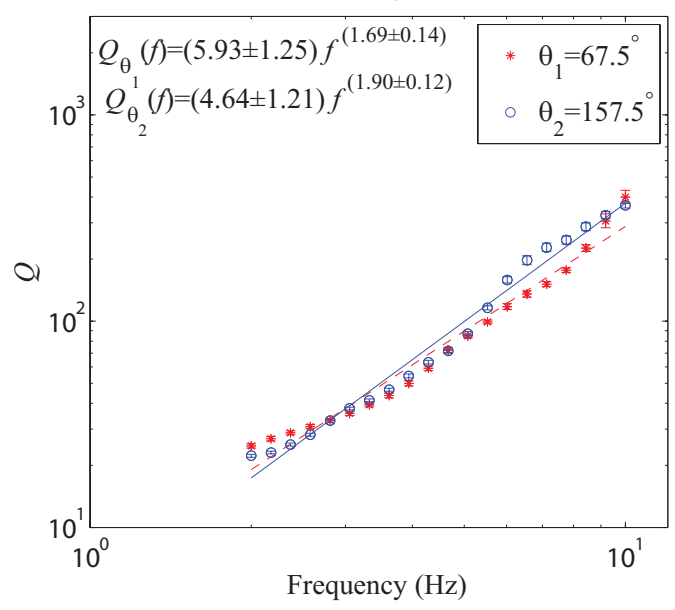

Figure 4.6 Estimates of homogenous $Q$ models for $\mathrm{S}$-waves obtained from the E-W, N-S and all rotational displacement spectra. The error bars denote $2 \sigma$ confidence interval 


\subsubsection{Two-layer $Q$ model}

To determine the depth of influence on the distance range of the amplitude decay function, we compute a raytracing model using velocity models (Table 4.2) derived from a seismic tomography study in the southernmost part of the TVZ (Rowlands et al., 2005). We find from raytracing using Mac Raytracer software (Luetgert, 1992) that the maximum depth of the rays for earthquakes recorded at distances $5-25 \mathrm{~km}$ is $\sim 9 \mathrm{~km}$ (Fig. 4.7). The distance range 5-25 km is defined based on the change of rate of amplitude decay observed on the amplitude decay curves. Therefore, it is reasonable to say that the first segment of the decay function $(5-25 \mathrm{~km})$ is associated with attenuation processes at depths less than 9 $\mathrm{km}$.

Table 4.2 Raytracing velocity models for $S$-waves in the southernmost part of the TVZ

\begin{tabular}{ll}
\hline$V_{S}(\mathrm{~km} / \mathrm{sec})$ & Depth $(\mathrm{km})$ \\
\hline 1.875 & 0 \\
2 & 3 \\
2.47 & 6 \\
3.23 & 9 \\
3.5 & 12 \\
3.88 & 30 \\
\hline
\end{tabular}

Figure 4.8 shows the upper crust $Q$ model computed from those seismograms with hypocentral distances of 5-25 km. $Q_{N}$ tends to be slightly higher than $Q_{E}$ at high frequencies, while all other horizontal component models yield insignificant differences between the two components in the frequency considered. These results suggest that the shear wave attenuation is only weakly anisotropic in the upper crust. We observe that the $Q$ values for the two-pair components of the R-T $Q$ model also show a slight difference in $Q$ at high frequencies $(f>7 \mathrm{~Hz})$, although the difference between $Q_{R}$ and $Q_{T}$ is within the statistical uncertainties of each estimate (Fig. 4.8). In general, the upper crust $Q$ model exhibits strong frequency dependence for the whole frequency range of interest marked by a high value of $\alpha$ (frequency-dependency coefficient) (Table 4.3). However, the north component of the E-N $Q$ model in the $8-10 \mathrm{~Hz}$ frequency band shows a weak frequency 


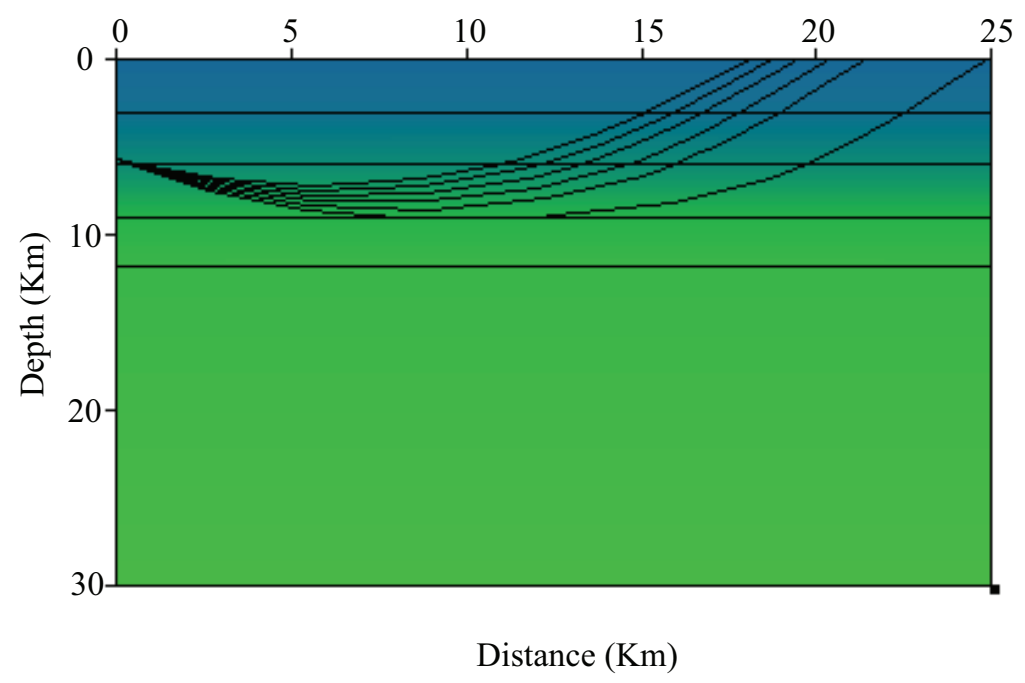

Figure 4.7 Rays propagating beneath the TVZ from a source at $5.6 \mathrm{~km}$ depth. The $5.6 \mathrm{~km}$ depth is the average hypocentral depth of the earthquakes shown in Fig. 3.7.

dependence with large uncertainties. The large error estimates are due to the effect of geometrical spreading correction on the the first segment (hypocentral distance $<25 \mathrm{~km}$ ) of attenuation function causing large uncertainties when applying linear least square fitting to estimate the slope.

Table 4.3 Summary of upper crust $Q$ model $\left(Q=Q_{0} f^{\alpha}\right)$ with $2 \sigma$ confidence level for the TVZ data

\begin{tabular}{ccccc}
\hline Orthogonal components & $Q_{0_{1}}$ & $\alpha_{1}$ & $Q_{0_{2}}$ & $\alpha_{2}$ \\
\hline N-E & $4.76 \pm 1.35$ & $2.10 \pm 0.19$ & $8.62 \pm 1.19$ & $1.58 \pm 0.11$ \\
NNE-ESE & $7.05 \pm 1.17$ & $1.69 \pm 0.10$ & $7.55 \pm 1.20$ & $1.61 \pm 0.12$ \\
NE-SE & $8.27 \pm 1.16$ & $1.56 \pm 0.09$ & $6.28 \pm 1.23$ & $1.74 \pm 0.13$ \\
ENE-SSE & $8.89 \pm 1.14$ & $1.49 \pm 0.09$ & $5.28 \pm 1.25$ & $1.87 \pm 0.14$ \\
R-T & $6.37 \pm 1.22$ & $1.77 \pm 0.13$ & $7.31 \pm 1.19$ & $1.63 \pm 0.11$ \\
\hline
\end{tabular}



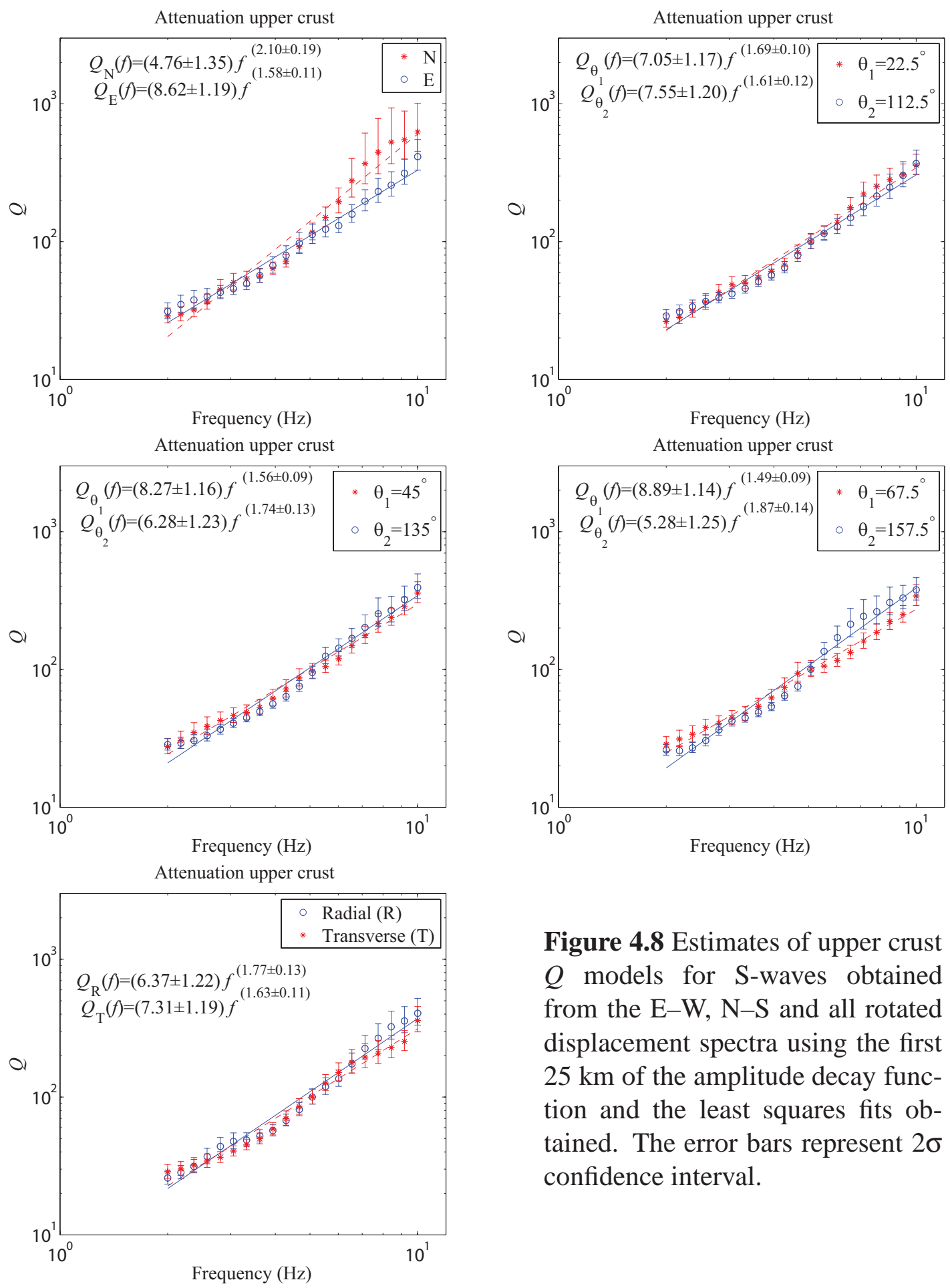

Figure 4.8 Estimates of upper crust $Q$ models for $\mathrm{S}$-waves obtained from the E-W, N-S and all rotated displacement spectra using the first $25 \mathrm{~km}$ of the amplitude decay function and the least squares fits obtained. The error bars represent $2 \sigma$ confidence interval. 

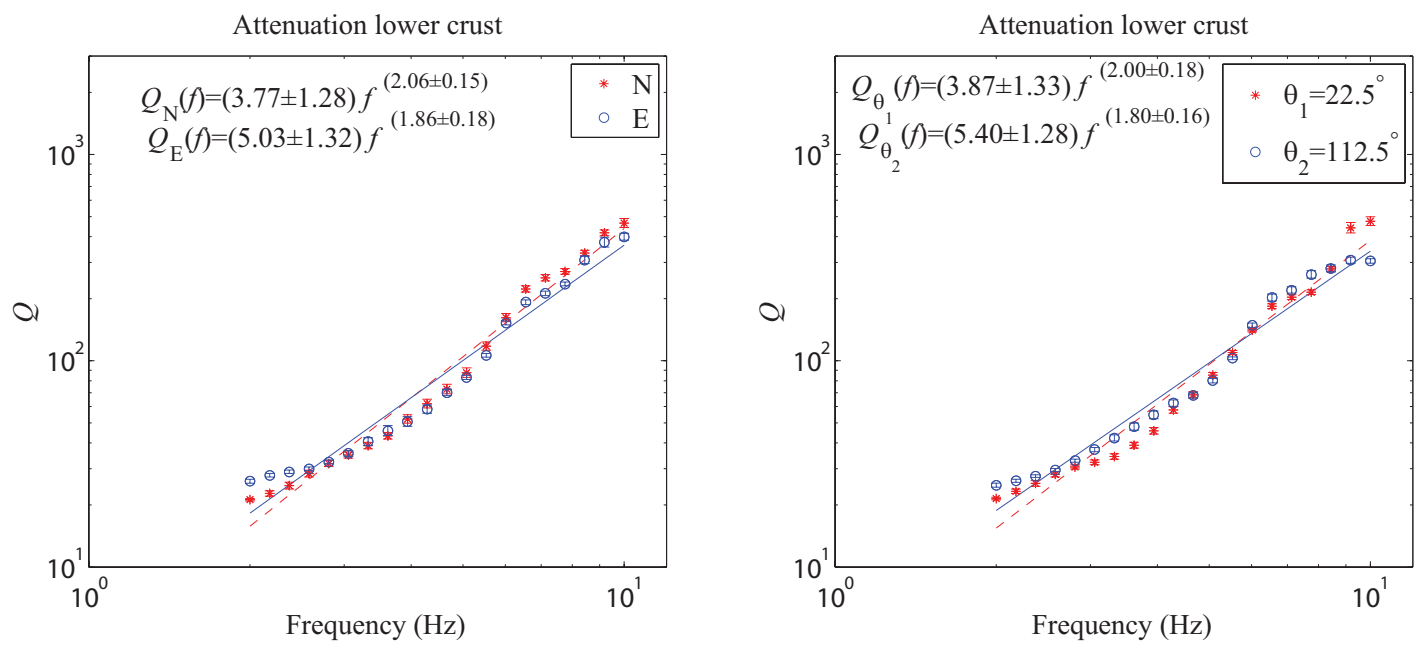

Attenuation lower crust

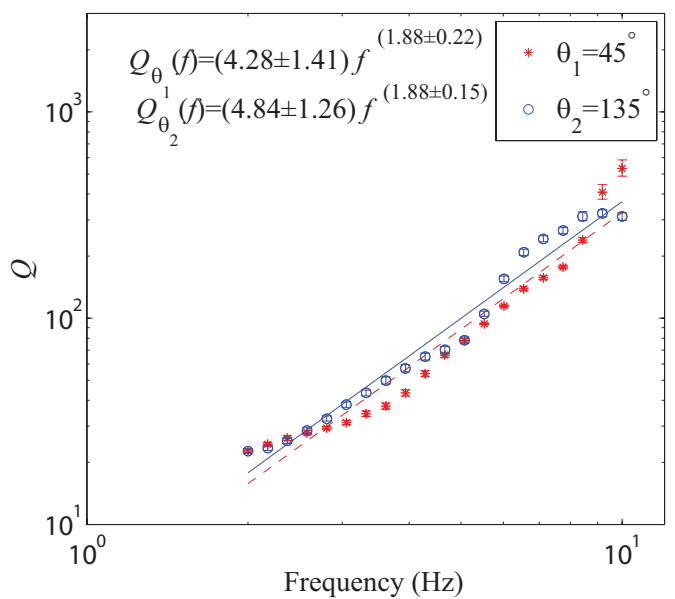

Attenuation lower crust

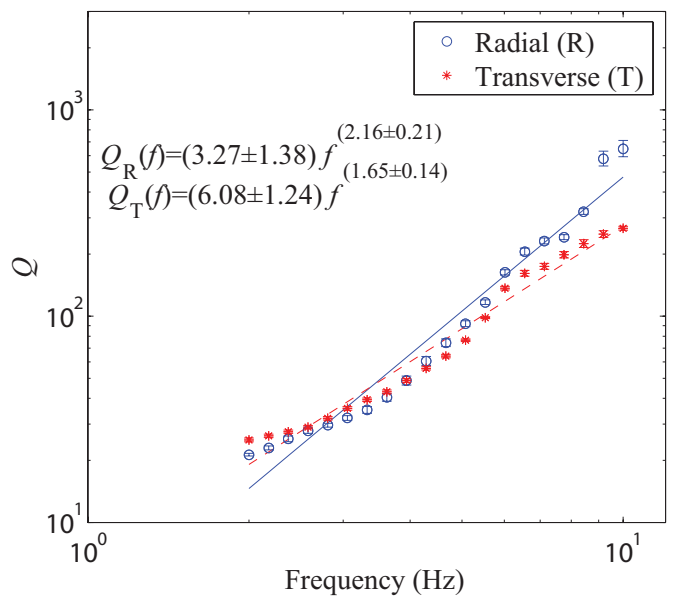

Figure 4.9 Estimates of $Q$ obtained for all rotational S-waves using the $25-52 \mathrm{~km}$ segment of the attenuation function and the least squares fits obtained (solid and dashed lines). The error bars represent $2 \sigma$ confidence interval 
The resulting estimate of lower crust $Q$ models (depth $>9 \mathrm{~km}$ calculated for the distance range $25-52$ of the decay function) for two horizontal components are depicted in Fig. 4.9. In general, the lower crust $Q$ model exhibits $Q$ values lower than those for the upper crust $Q$ model (Tables 4.3 and 4.4). At the rotation angle $\theta=45^{\circ}$, the estimates of $Q$ for the S-waves in the $135^{\circ}$ direction are generally higher than those at $45^{\circ}$ direction, particularly in the 3-8 Hz frequency range. The difference of $Q$ values between a pair of orthogonal components also can be seen in the $Q$ model for radial and transverse components, indicating greater attenuation at high frequencies ( $\mathrm{f}>5 \mathrm{~Hz}$ ) for $\mathrm{S}$-waves in the transverse direction than for those in radial components. However, the $Q$ values for model $\theta=135^{\circ}$ between $7-10 \mathrm{~Hz}$ becomes approximately constant with frequency.

Table 4.4 Summary of lower crust $Q$ model $\left(Q=Q_{0} f^{\alpha}\right)$ with $2 \sigma$ confidence level for the TVZ data

\begin{tabular}{ccccc}
\hline Orthogonal components & $Q_{0_{1}}$ & $\alpha_{1}$ & $Q_{0_{2}}$ & $\alpha_{2}$ \\
\hline N-E & $3.77 \pm 1.28$ & $2.06 \pm 0.15$ & $5.03 \pm 1.32$ & $1.86 \pm 0.18$ \\
NNE-ESE & $3.87 \pm 1.33$ & $2.00 \pm 0.18$ & $5.40 \pm 1.28$ & $1.80 \pm 0.16$ \\
NE-SE & $4.28 \pm 1.41$ & $1.88 \pm 0.22$ & $4.84 \pm 1.26$ & $1.88 \pm 0.15$ \\
ENE-SSE & $4.79 \pm 1.41$ & $1.83 \pm 0.22$ & $4.17 \pm 1.27$ & $1.97 \pm 0.15$ \\
R-T & $3.27 \pm 1.38$ & $2.16 \pm 0.21$ & $6.08 \pm 1.24$ & $1.65 \pm 0.14$ \\
\hline
\end{tabular}




\subsection{Q model outside the TVZ}

The distribution of orientations of the radial and transverse components from the nonTVZ data described in Section 3.8 are depicted in the rose diagrams below. Figure 4.10 indicates that the most frequent directions for the radial component are in the direction ranges $70^{\circ}-80^{\circ}$ and $110^{\circ}-120^{\circ}$, while the transverse component has prominent directions at orthogonal directions.
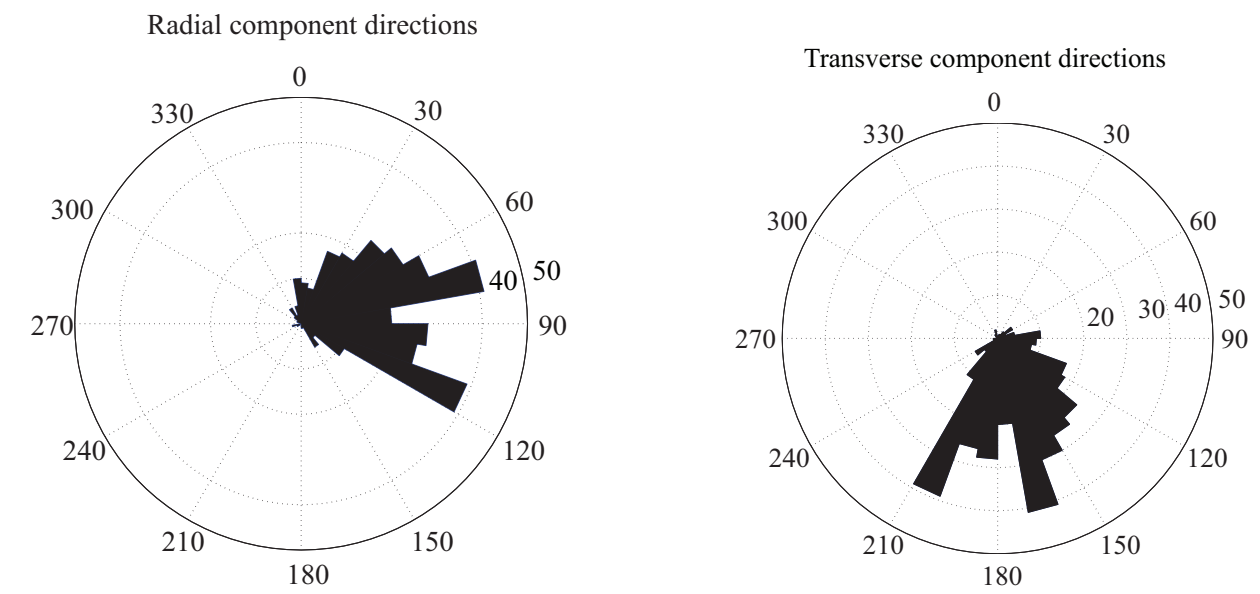

Figure 4.10 Rose diagrams showing the directions of the radial and transverse components for the non-TVZ data based on earthquake and station locations

We only measure the $Q$ model using a homogeneous model, because we do not see any significant changes of the decay rate with distance (Fig. 4.11). Figure 4.12 shows the results of attenuation for the regional paths outside the TVZ (see Fig. 3.8). In general, $Q$ is higher and the frequency dependence of $Q$ is more complex for $\mathrm{S}$ waves outside the TVZ (Table 4.5 and Fig. 4.12). In this Figure, $Q$ values for all rotated seismograms show a weak dependence of frequency on the frequency range around 2-7 Hz. The trend above $7 \mathrm{~Hz}$ becomes more frequency-dependent. The NE-SW direction $\left(45^{\circ}\right)$ has high anisotropy at low frequencies compared to other horizontal directions. By fitting straight lines to the points within the $2-12 \mathrm{~Hz}$ range for the NE and SE directions, we find that the $Q$ frequency dependence of $\mathrm{S}$ waves can be approximated with the functions $Q_{S_{N E}}(f)=$ $(50.93 \pm 1.18) f^{(0.20 \pm 0.10)}$ and $Q_{S_{S E}}(f)=(22.60 \pm 1.10) f^{(0.53 \pm 0.06)}$. 
Table 4.5 Summary of homogeneous $Q$ model $\left(Q=Q_{0} f^{\alpha}\right)$ with $2 \sigma$ confidence level for the non-TVZ data

\begin{tabular}{ccccc}
\hline Orthogonal components & $Q_{0_{1}}$ & $\alpha_{1}$ & $Q_{0_{2}}$ & $\alpha_{2}$ \\
\hline N-E & $56.21 \pm 1.14$ & $0.18 \pm 0.08$ & $46.57 \pm 1.21$ & $0.29 \pm 0.11$ \\
NNE-ESE & $111.57 \pm 1.38$ & $-0.15 \pm 0.19$ & $39.59 \pm 1.21$ & $0.37 \pm 0.11$ \\
NE-SE & $50.93 \pm 1.18$ & $0.20 \pm 0.10$ & $22.60 \pm 1.10$ & $0.53 \pm 0.06$ \\
ENE-SSE & $36.08 \pm 1.14$ & $0.35 \pm 0.08$ & $23.97 \pm 1.10$ & $0.49 \pm 0.06$ \\
R-T & $98.37 \pm 1.32$ & $-0.05 \pm 0.17$ & $30.63 \pm 1.20$ & $0.39 \pm 0.11$ \\
\hline
\end{tabular}



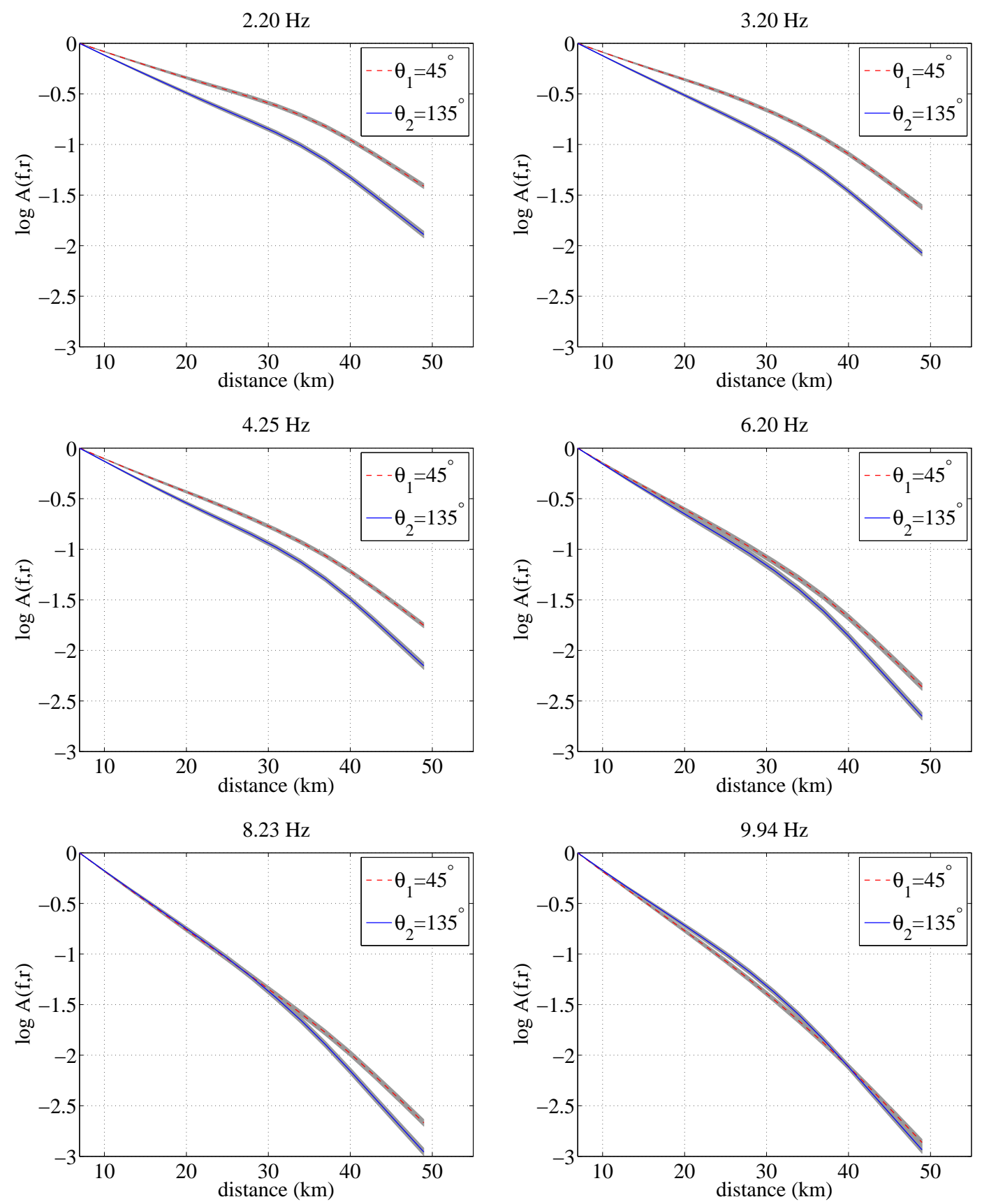

Figure 4.11 Amplitude decay functions versus hypocentral distance for rotation $45^{\circ}$ at six frequencies obtained from the non-TVZ data. The dashed red and blue lines represent the mean of 200 bootstrap samples and the gray shaded area represents the mean $\pm 2 \sigma$. 

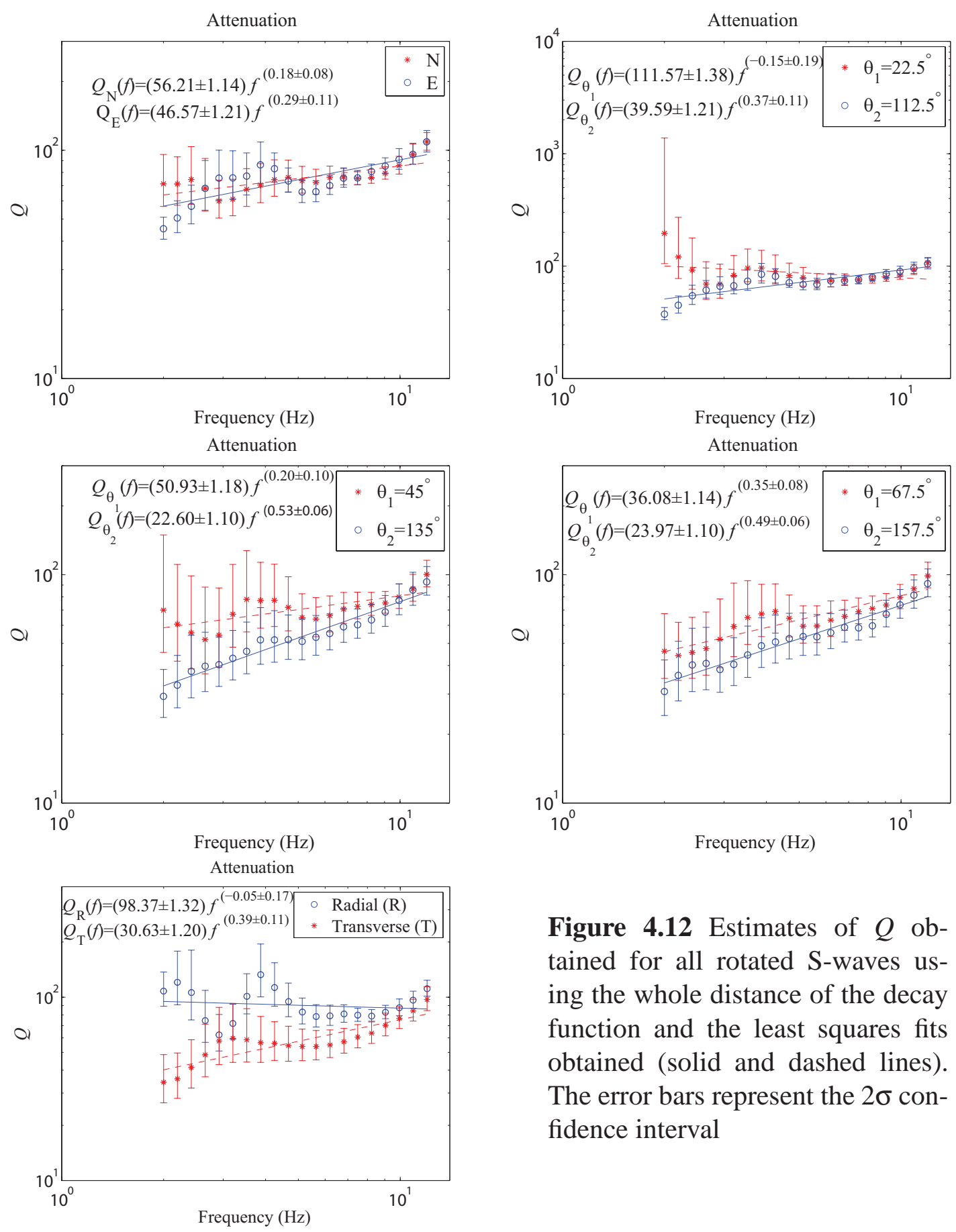

Figure 4.12 Estimates of $Q$ obtained for all rotated S-waves using the whole distance of the decay function and the least squares fits obtained (solid and dashed lines). The error bars represent the $2 \sigma$ confidence interval 


\subsection{Source and directional site effects}

The major focus of this work is attenuation anisotropy. However, source and site effects result as a by product. We examine the source and site effects by separating source and site amplification functions using the second inversion of the non-parametric GIT method, as described in Section 3.1.1. We impose the constraint in equation 3.17 that the average of the logarithmic site terms is equal to zero to eliminate the linear dependence between the source and site functions. However, in the inversion of the TVZ data we do not use all seismic stations as reference sites because there are more than five stations containing less than 10 earthquakes (Table 4.6). Using all stations as the reference sites caused the matrix inversion to be unstable. So instead, we select stations containing at least 10 earthquakes as reference sites for the TVZ data. For the non-TVZ data, however, we include all stations for the reference sites because most of them contain more than 10 events (Table 4.6).

Table 4.6 Number of earthquakes recorded by GeoNet stations around the TVZ

\begin{tabular}{|l|l|l|l|}
\hline Name & Code & \multicolumn{2}{|c|}{ Number of earthquakes } \\
\cline { 3 - 4 } & & The TVZ & The non-TVZ \\
\hline Dome Shelter & DRZ & 2 & 18 \\
Far West & FWVZ & 11 & 29 \\
Karewarewa & KRVZ & 47 & 11 \\
Moawhango & MOVZ & 8 & 25 \\
Mangateitei & MTVZ & 6 & 23 \\
Ngauruhoe & NGZ & 39 & 16 \\
Oturere & OTVZ & 24 & 13 \\
Pokaka & PKVZ & 3 & 6 \\
Turoa & TRVZ & 4 & 12 \\
Tukino & TUVZ & 25 & 29 \\
Taurewa & TWVZ & 28 & 34 \\
Wahianoa & WNVZ & 6 & 38 \\
Whakapapa & WPVZ & 42 & 42 \\
West Tongariro & WTVZ & 71 & 42 \\
\hline
\end{tabular}




\subsubsection{Source effects}

The source functions obtained from the second inversion can be written in a functional form with a single corner frequency proposed by Boatwright (1978):

$$
S(f)=\Omega_{0} /\left[1+\left(f / f_{c}\right)^{2 \gamma}\right]^{1 / 2}
$$

Here $\Omega_{0}$ is the low-frequency amplitude level, $f_{c}$ is the corner frequency and $\gamma$ represents the displacement spectral falloff above the corner frequency. The corner frequencies are determined directly from the measured source functions. Then, the best values of the decay rate $\gamma$ are determined by fitting the theoretical model with the observed model. In this study, we compute manually source parameters of 41 selected events from the TVZ data.

Figure 4.13 displays a sample of six of the resulting source functions obtained from the second step inversion for events within the magnitude range of 2.0-3.2. The solid lines correspond to the inverted source function in the east and north components and the dashed lines correspond to the optimal model fit. In general, the theoretical models match well the displacement spectra obtained from the inversion and both components show similar amplitude-frequency trends. However, for a given frequency, the source function amplitude for the north component is sometimes higher than that for the east component.

The selected source terms in Table 4.7 provide corner frequencies ranging from 2.2 to 4.4 Hz and a high spectral fall-off $\gamma$ ranging from 1.9 to 4.9 for the east component, while the north component data has corner frequencies of $2.4-3.9 \mathrm{~Hz}$ and spectral fall-off of 2.4-4.9. These results indicate that all spectra may decay close to $\omega^{-2}$ and $\omega^{-3}$ or greater. 

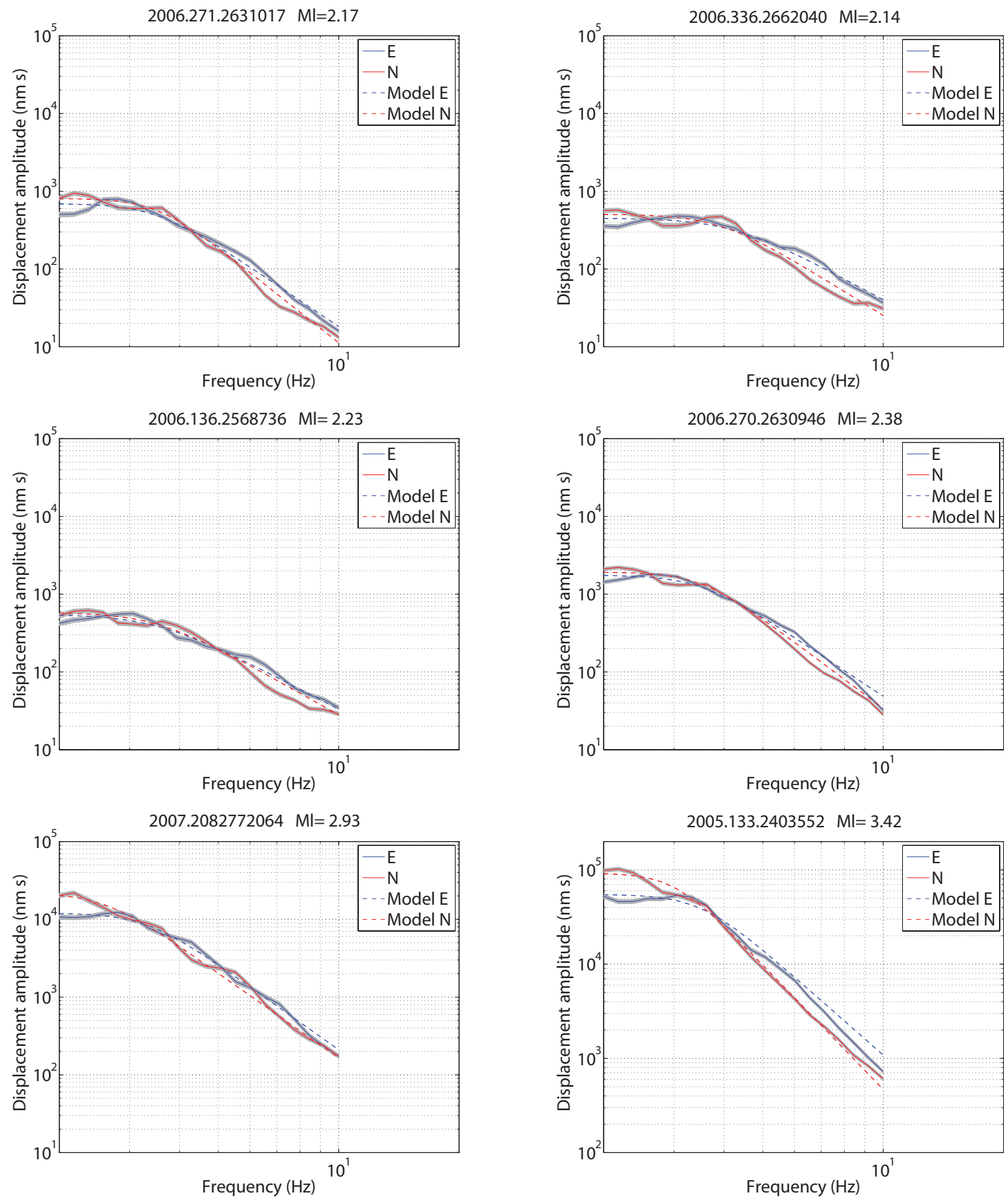

Figure 4.13 The selected source function obtained from the second inversion. The dashed line corresponds to the theoretical model. The gray shaded area represents the mean $\pm 2 \sigma$. 
Table 4.7 Examples of source parameters calculated for selected TVZ events with $95 \%$ confidence level

\begin{tabular}{|c|c|c|c|c|c|c|c|}
\hline Event & $\Omega_{0 E}(\mathbf{n m} \mathbf{~ s})$ & $\Omega_{0 N}(\mathbf{n m ~ s})$ & $f_{c E}$ & $f_{c N}$ & $\gamma_{E}$ & $\gamma_{N}$ & $\mathbf{M I}^{1}$ \\
\hline 2004.250 .2290416 & $13640 \pm 970$ & $20510 \pm 950$ & 3.0 & 2.5 & $3.2 \pm 0.61$ & $4.4 \pm 0.50$ & 2.86 \\
2004.357 .2342078 & $1841 \pm 280$ & $2422 \pm 202$ & 3.4 & 3.5 & $3.3 \pm 1.54$ & $4.5 \pm 1.38$ & 2.23 \\
2005.022 .2558323 & $1042 \pm 28$ & $1319 \pm 99$ & 3.4 & 3.3 & $2.9 \pm 0.23$ & $3.6 \pm 0.84$ & 2.20 \\
2005.133 .2403552 & $55020 \pm 3680$ & $92430 \pm 5560$ & 3.5 & 3 & $3.7 \pm 0.82$ & $4.4 \pm 0.82$ & 3.42 \\
2005.289 .2473535 & $938.8 \pm 51.6$ & $1609 \pm 58$ & 3.6 & 2.9 & $3.1 \pm 0.53$ & $3.8 \pm 0.38$ & 2.30 \\
2006.023 .2517149 & $808.1 \pm 105.6$ & $847.8 \pm 35.6$ & 3.3 & 2.9 & $2.9 \pm 0.86$ & $2.7 \pm 0.28$ & 2.11 \\
2006.061 .2532957 & $1058 \pm 61.7$ & $1035 \pm 91.6$ & 2.7 & 3.1 & $2.6 \pm 0.34$ & $3.8 \pm 1.01$ & 2.11 \\
2006.106 .2554479 & $2499 \pm 150$ & $4292 \pm 184$ & 3.5 & 2.9 & $2.6 \pm 0.47$ & $3.0 \pm 0.33$ & 2.49 \\
2006.136 .2568736 & $550.1 \pm 39.9$ & $582.6 \pm 34.5$ & 3.5 & 3.5 & $2.7 \pm 0.57$ & $2.9 \pm 0.52$ & 2.23 \\
2006.270 .2630946 & $1755 \pm 90$ & $1919 \pm 129$ & 3.5 & 3.5 & $3.4 \pm 0.57$ & $3.8 \pm 0.88$ & 2.38 \\
2006.271 .2631017 & $693.6 \pm 68.9$ & $808.1 \pm 49$ & 3.5 & 3.5 & $3.5 \pm 1.11$ & $4.1 \pm 0.86$ & 2.17 \\
2006.327 .2657786 & $886.8 \pm 150$ & $1664 \pm 134$ & 4.3 & 3.2 & $4.6 \pm 3.43$ & $3.3 \pm 0.77$ & 2.28 \\
2006.335 .2661705 & $6388 \pm 421$ & $8226 \pm 499$ & 3.2 & 2.9 & $2.8 \pm 0.51$ & $3.1 \pm 0.49$ & 2.81 \\
2006.336 .2662040 & $450.8 \pm 30.5$ & $506.6 \pm 44$ & 4.2 & 3.9 & $2.8 \pm 0.66$ & $3.2 \pm 0.94$ & 2.14 \\
2007.009 .2680142 & $1465 \pm 130$ & $1593 \pm 89$ & 3.3 & 3.2 & $3.0 \pm 0.60$ & $3.1 \pm 0.50$ & 2.26 \\
2007.061 .2704153 & $595.9 \pm 32.4$ & $639.6 \pm 58.8$ & 3.7 & 3.5 & $2.3 \pm 0.37$ & $2.6 \pm 0.70$ & 2.10 \\
2007.089 .2717771 & $8077 \pm 547$ & $12590 \pm 540$ & 2.9 & 2.5 & $3.2 \pm 0.57$ & $3.9 \pm 0.39$ & 2.87 \\
2007.091 .2718437 & $948.5 \pm 34.2$ & $1302 \pm 75$ & 4.0 & 3.6 & $3.4 \pm 0.44$ & $4.3 \pm 0.92$ & 2.14 \\
2007.098 .2722077 & $598.6 \pm 81.4$ & $939.6 \pm 170.1$ & 3.5 & 3.1 & $4.3 \pm 2.10$ & $4.1 \pm 2.32$ & 2.22 \\
2007.207 .2771378 & $1595 \pm 85$ & $2988 \pm 133$ & 3.0 & 2.4 & $3.0 \pm 0.42$ & $3.3 \pm 0.30$ & 2.33 \\
2007.207 .2771402 & $3456 \pm 199$ & $6225 \pm 186$ & 3.3 & 2.9 & $2.8 \pm 0.45$ & $3.5 \pm 0.28$ & 2.63 \\
2007.207 .2771473 & $4481 \pm 232$ & $5195 \pm 262$ & 3.3 & 3.1 & $2.8 \pm 0.40$ & $3.2 \pm 0.45$ & 2.70 \\
2007.207 .2771475 & $1052 \pm 69.4$ & $1515 \pm 84$ & 3.2 & 2.5 & $2.9 \pm 0.54$ & $2.7 \pm 0.32$ & 2.14 \\
2007.207 .2771553 & $7156 \pm 308$ & $10180 \pm 563$ & 3.1 & 3 & $2.6 \pm 0.30$ & $3.1 \pm 0.46$ & 3.11 \\
2007.208 .2771980 & $4098 \pm 312$ & $6247 \pm 229$ & 3.2 & 2.6 & $3.3 \pm 0.73$ & $3.4 \pm 0.29$ & 2.48 \\
2007.208 .2772028 & $2438 \pm 87$ & $3072 \pm 197$ & 2.8 & 2.9 & $3.2 \pm 0.29$ & $4.9 \pm 1.00$ & 2.35 \\
\hline & & & & $C o n t i n u e d$ on next page \\
\hline
\end{tabular}


Table 4.7 - continued from previous page

\begin{tabular}{|c|c|c|c|c|c|c|c|}
\hline Event & $\Omega_{E}(\mathbf{n m} \mathbf{~ s})$ & $\Omega_{0 N}(\mathbf{n m} \mathbf{~ s})$ & $f_{c E}$ & $f_{c N}$ & $\gamma_{E}$ & $\gamma_{N}$ & MI $^{1}$ \\
\hline 2007.208 .2772064 & $11980 \pm 610$ & $21740 \pm 1060$ & 3.3 & 2.6 & $3.6 \pm 0.59$ & $3.6 \pm 0.42$ & 2.93 \\
2007.209 .2772336 & $621.5 \pm 67.9$ & $747.6 \pm 23$ & 3.3 & 3.1 & $3.3 \pm 1.07$ & $3.3 \pm 0.29$ & 2.02 \\
2007.264 .2797040 & $3204 \pm 289$ & $3792 \pm 341$ & 3.5 & 3.2 & $2.8 \pm 0.77$ & $3.1 \pm 0.81$ & 2.59 \\
2007.320 .2823239 & $936.6 \pm 49.7$ & $1344 \pm 126$ & 3.8 & 3.3 & $3.2 \pm 0.56$ & $3.4 \pm 0.97$ & 2.34 \\
2008.001 .2844837 & $565.8 \pm 33.2$ & $693.5 \pm 24.2$ & 2.2 & 2.6 & $1.9 \pm 0.18$ & $2.7 \pm 0.20$ & 2.19 \\
2008.050 .2867955 & $733.5 \pm 158.6$ & $819.6 \pm 82.6$ & 4.4 & 3.8 & $2.1 \pm 1.29$ & $2.4 \pm 0.75$ & 2.22 \\
2008.157 .2918616 & $5313 \pm 574$ & $8992 \pm 461$ & 3.3 & 3 & $3.6 \pm 1.23$ & $4.8 \pm 0.80$ & 2.26 \\
2008.197 .2938750 & $563.4 \pm 42$ & $890.6 \pm 47.9$ & 3.8 & 2.7 & $3.3 \pm 0.75$ & $3.1 \pm 0.40$ & 2.13 \\
2008.208 .2944198 & $1027 \pm 54$ & $1268 \pm 115$ & 3.2 & 2.9 & $2.3 \pm 0.32$ & $2.4 \pm 0.53$ & 2.17 \\
2008.211 .2945663 & $4685 \pm 372$ & $4932 \pm 537$ & 3.1 & 3.2 & $3.2 \pm 0.70$ & $3.9 \pm 1.31$ & 2.62 \\
2008.223 .2951293 & $7136 \pm 776$ & $7504 \pm 760$ & 3.1 & 3.1 & $3.6 \pm 1.13$ & $3.9 \pm 1.21$ & 2.52 \\
2008.225 .2952218 & $5352 \pm 168$ & $6742 \pm 802$ & 3.2 & 3.2 & $3.7 \pm 0.35$ & $4.5 \pm 1.81$ & 2.46 \\
2008.244 .2961706 & $5280 \pm 774$ & $7519 \pm 512$ & 3.2 & 3 & $3.8 \pm 1.70$ & $4.7 \pm 1.04$ & 2.30 \\
2009.003 .3024650 & $1159 \pm 61$ & $2320 \pm 49$ & 3.3 & 2.4 & $3.9 \pm 0.66$ & $3.6 \pm 0.16$ & 2.14 \\
2009.014 .3030120 & $724.6 \pm 50.5$ & $1099 \pm 68$ & 3.8 & 3.4 & $3.9 \pm 1.01$ & $4.2 \pm 0.89$ & 2.02 \\
\hline
\end{tabular}

\footnotetext{
${ }^{1}$ the magnitudes ML are defined according to the Richter definition of local magnitude (e.g. Stein and Wysession, 2003).
} 


\subsubsection{Directional site effects}

\section{The TVZ results}

The directional site functions of several stations obtained using the TVZ dataset in the second step of the inversion described in Section 3.15 are shown in Figure 4.14, 4.15 and 4.16. As expected, the sites containing more than 10 events generally have site amplifications close to one, as imposed by the constraint. Although the general trends of the curves are similar for all rotated data in each seismic station, their amplitudes differ for each direction. For instance, the general trend of amplification of all rotated data in station OTVZ decreases with increasing frequency. However, their amplitudes are different within a given frequency band (e.g. the amplification in the $45^{\circ}$ direction is lower than that at $157.5^{\circ}$ for frequencies less than $6 \mathrm{~Hz}$ ). Another interesting feature is that site amplification curves obtained for stations TRVZ and DRZ are not as smooth as those for other stations.
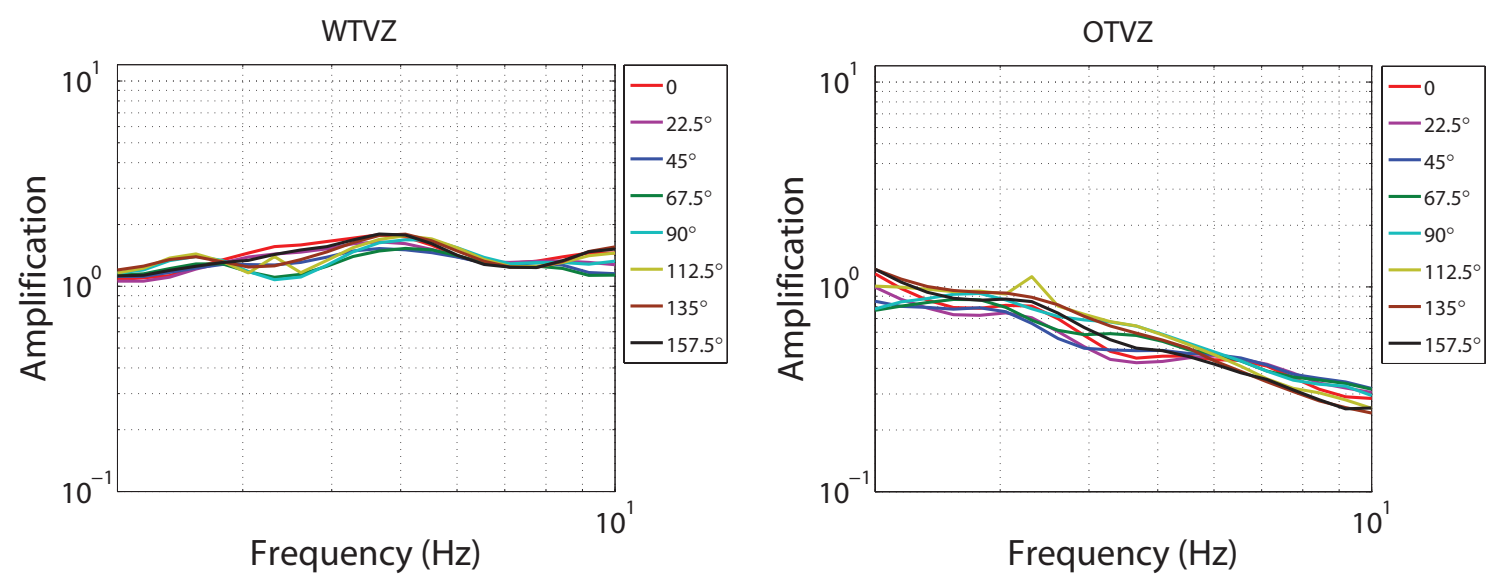

Figure 4.14 Directional site amplifications of the TVZ for stations WTVZ and OTVZ. 

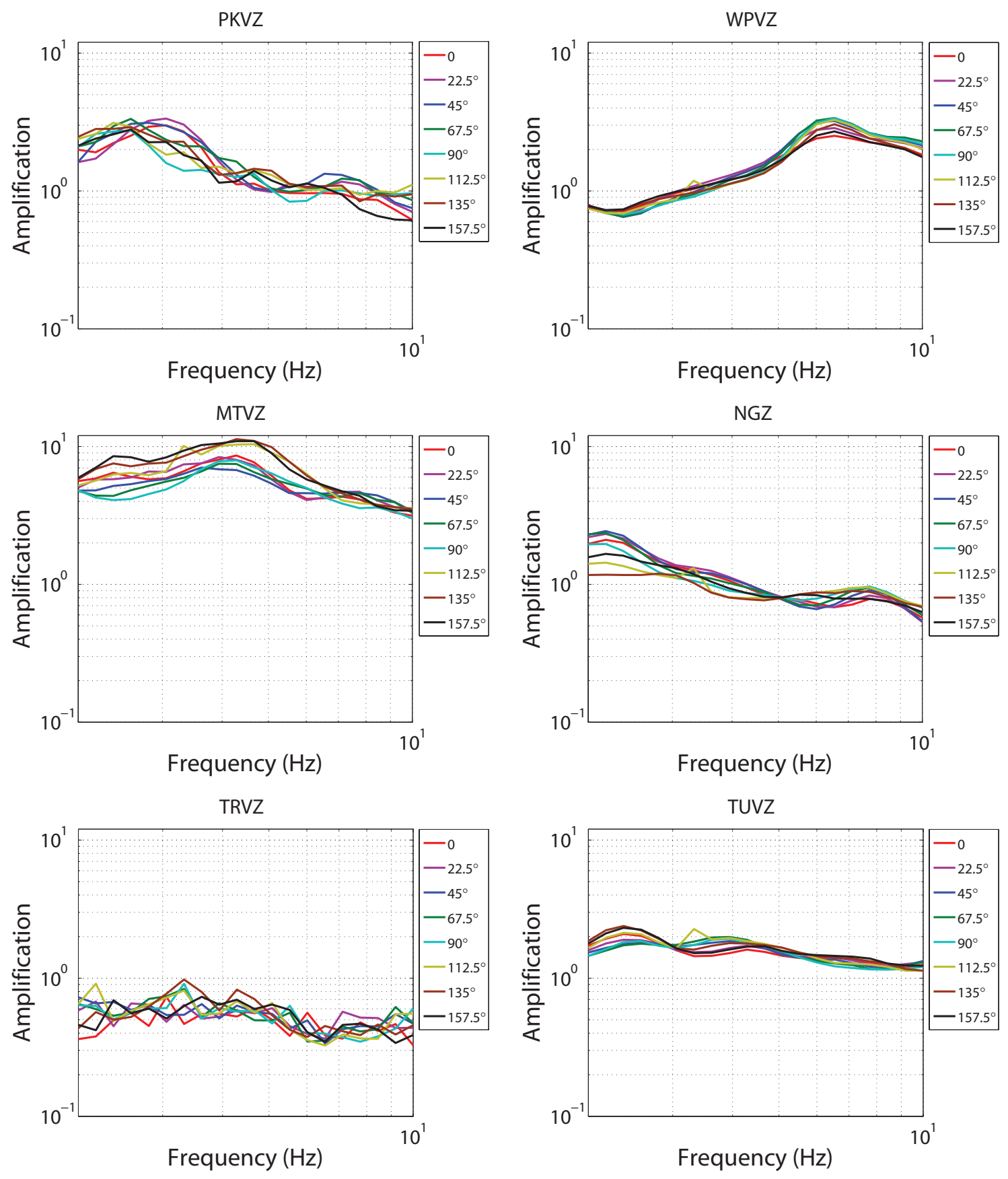

Figure 4.15 Directional site amplifications of the TVZ for stations PKVZ, WPVZ, MTVZ, NGZ, TRVZ and TUVZ. 

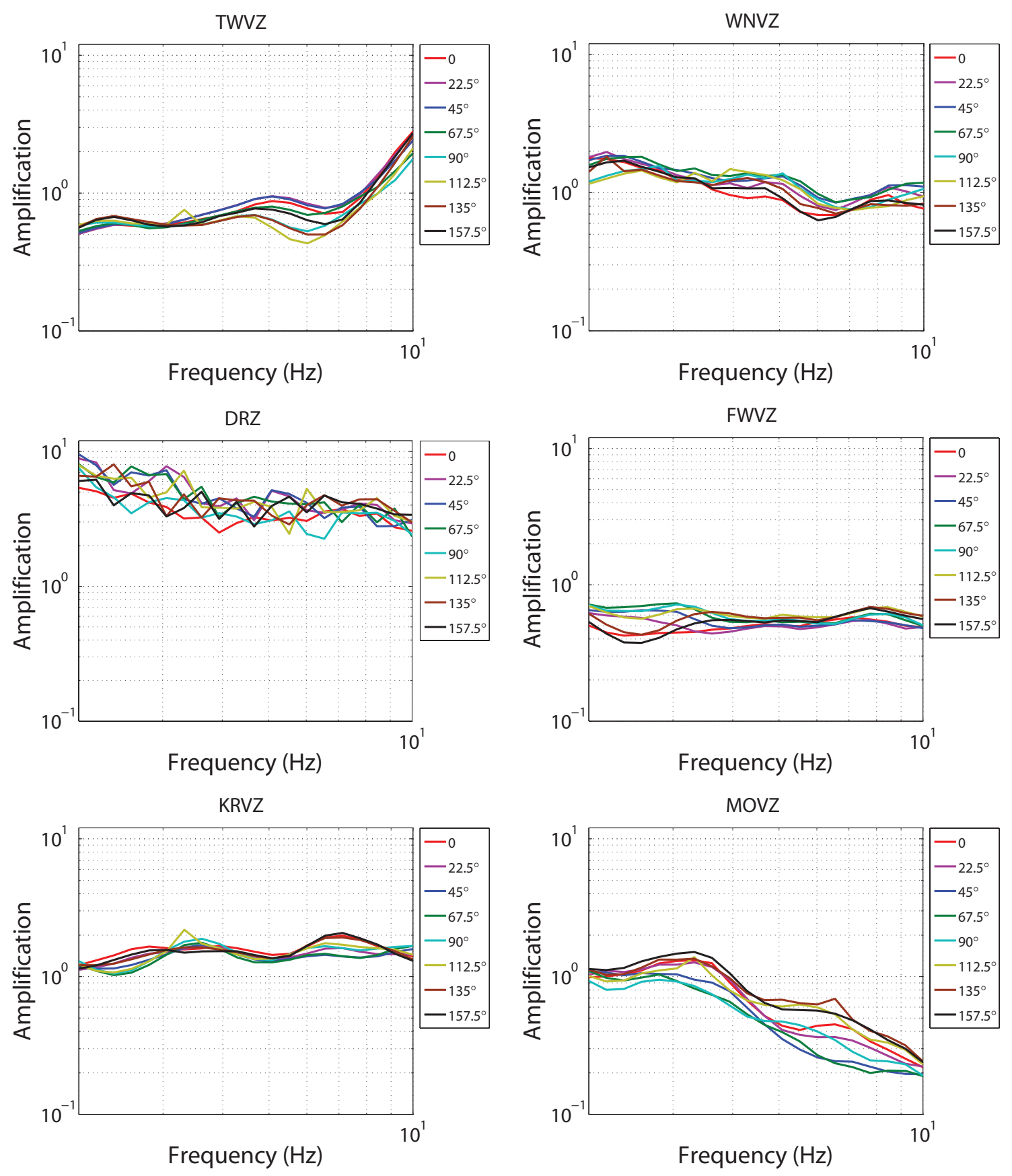

Figure 4.16 Directional site amplifications of the TVZ for stations TWVZ, WNVZ, DRZ, FWVZ, KRVZ and MOVZ. 
Figures 4.17 and 4.18 show the site amplifications of selected stations as functions of frequency and direction of polarization. The contours of the site amplification at station PKVZ and WPVZ exhibit peaks centered at 3.5 and $6.2 \mathrm{~Hz}$, respectively, while stations WTVZ and OTVZ do not show any significant peaks. At site PKVZ, the contouring confirms peak values of up to 4 in the directions of $0^{\circ}$ to $67.5^{\circ}$ without a broadening effect of the amplified frequency band. A peak value around 4 is also observed for station WPVZ in the directions of $45^{\circ}$ to $157.5^{\circ}$.

Figure 4.19 displays the directional site effects at 14 GeoNet stations around the TVZ. In general, contours of the geometric mean of the site amplification for some seismic stations exhibit the presence of a directional resonance. Most of the peak values of the site amplifications occur at low frequencies $(f<6 \mathrm{~Hz})$, except for stations WPVZ and TWVZ. The highest amplifications occur at station MTVZ for direction of $157.5^{\circ}$ with an amplitude around 10. Another interesting feature of the directional site effect is that the orientation of the directional resonance is found to be predominantly about $45^{\circ}$ and $157.5^{\circ}$. For instance, the resonance direction at sites MTVZ and TUVZ is around $157.5^{\circ}$, while stations NGZ and DRZ exhibit the resonance direction at $45^{\circ}$. A homogeneous pattern of site amplification is also found at the sites close to the summit of Ruapehu, namely TRVZ and FWVZ. 

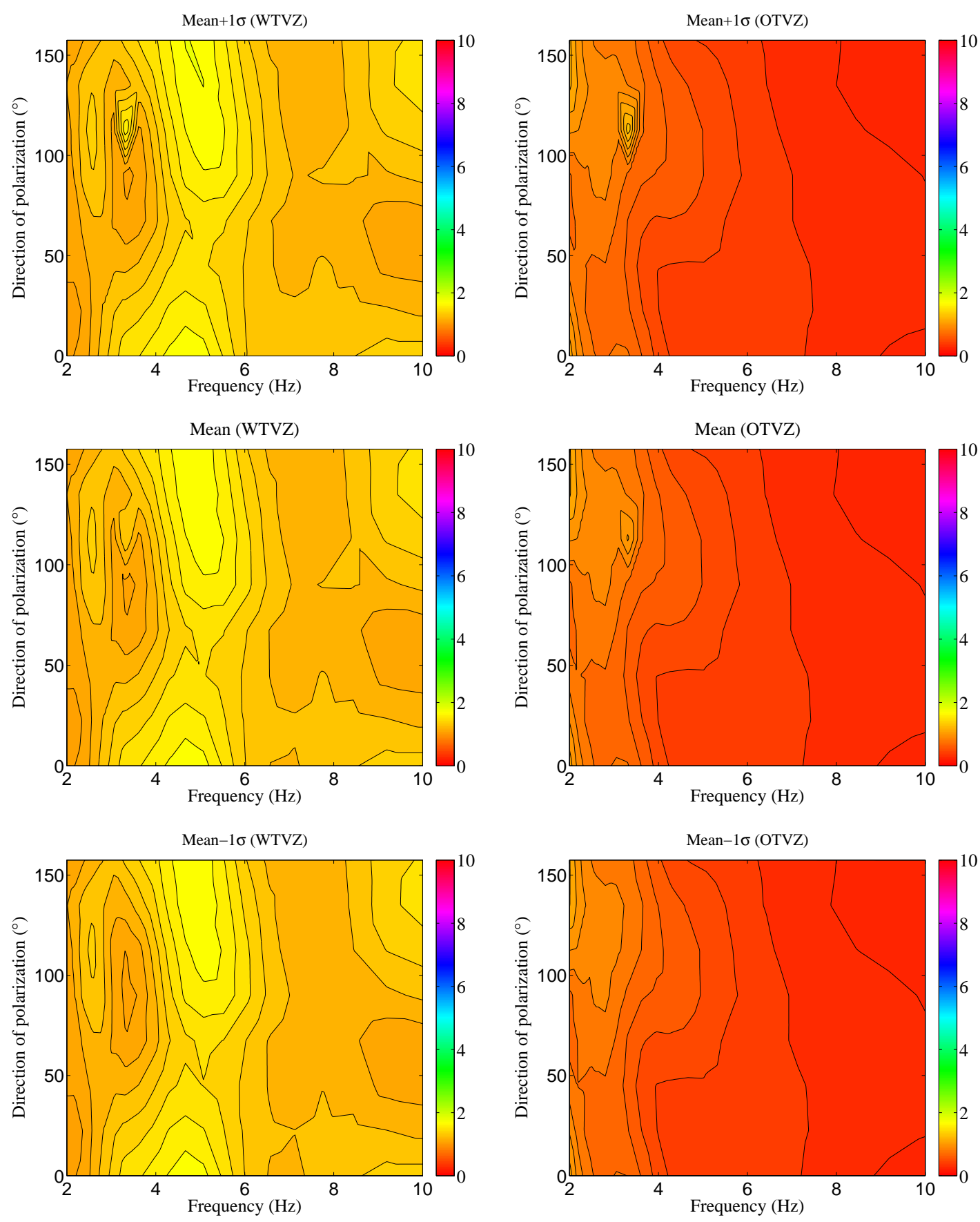

Figure 4.17 The directional site amplification as a function of frequency and direction of polarization obtained for stations OTVZ and WTVZ. The mid panel shows the mean of 200 bootstrap inversion of the directional site effect, while the top and bottom panels show the $\pm 1 \sigma$, respectively. 

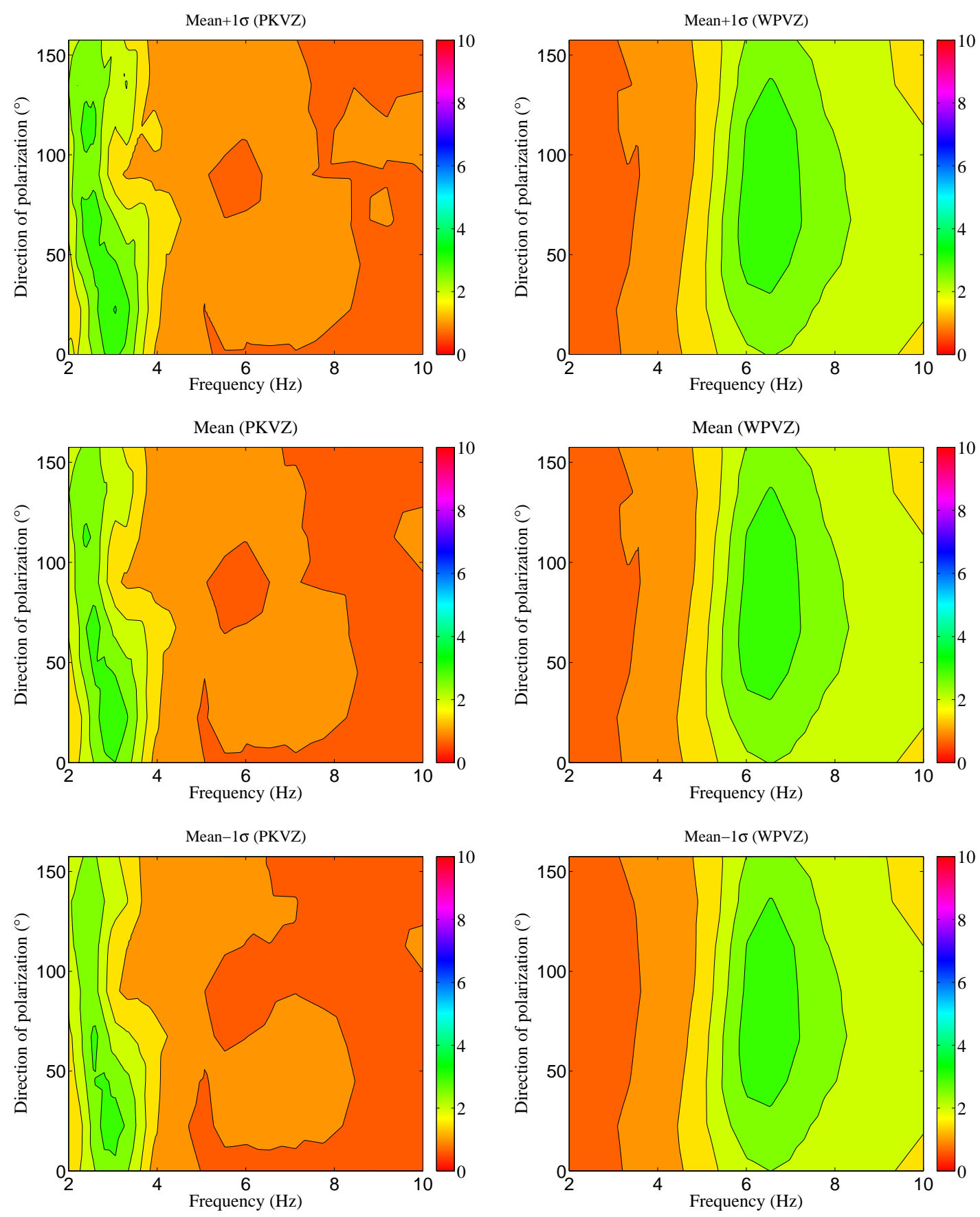

Figure 4.18 The directional site amplification as a function of frequency and direction of polarization obtained for stations PKVZ and WPVZ. The mid panel shows the mean of 200 bootstrap inversion of the directional site effect, while the top and bottom panels show the $\pm 1 \sigma$, respectively. 


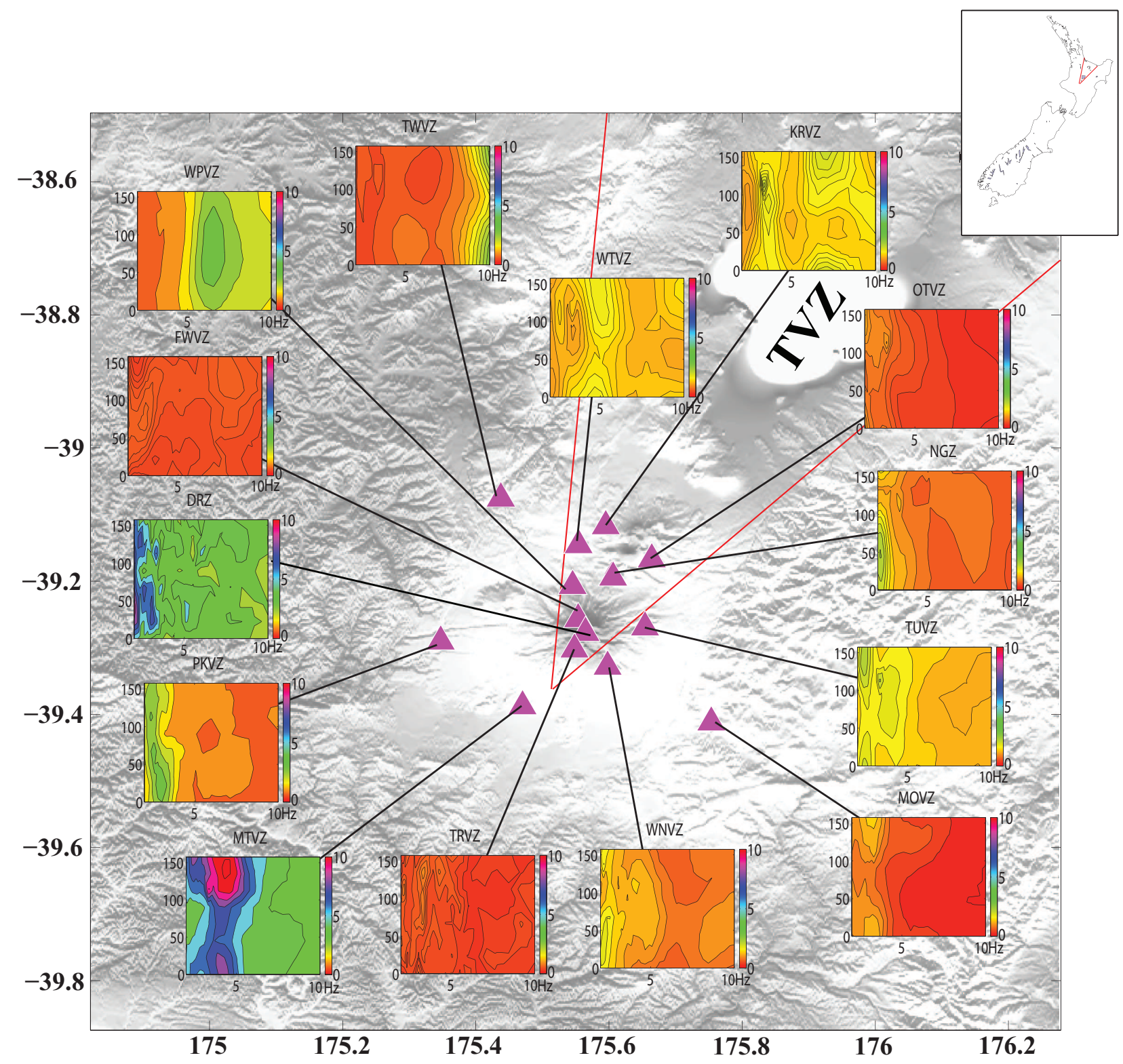

Figure 4.19 The directional site amplifications in the southernmost part of the TVZ using the TVZ dataset. 


\section{The non-TVZ results}

We invert the non-TVZ dataset separately in order to investigate possible directional dependence of site amplification on source location. Figure 4.20, 4.21 and 4.22 display the resulting site amplifications obtained from the inversion of the non-TVZ dataset. As expected, the directional site amplifications of the non-TVZ data are generally comparable to the previous estimates of the directional site function obtained from the TVZ data. The shape of the directional site functions from both datasets are very similar. However, the amplification values at stations DRZ, MTVZ and PKVZ for the TVZ data are higher than for the non-TVZ data.

Figure 4.23 shows that the directional resonance pattern of GeoNet stations around the TVZ are generally similar for both datasets. However, a comparison of Fig. 4.23 and 4.19 demonstrates that there are peaks for several stations (e.g. DRZ, MTVZ and PKVZ) for the TVZ model do not occur in the non-TVZ model. Furthermore, the average amplifications of the TVZ model at station DRZ, MTVZ and PKVZ are higher than those of the outside TVZ one.
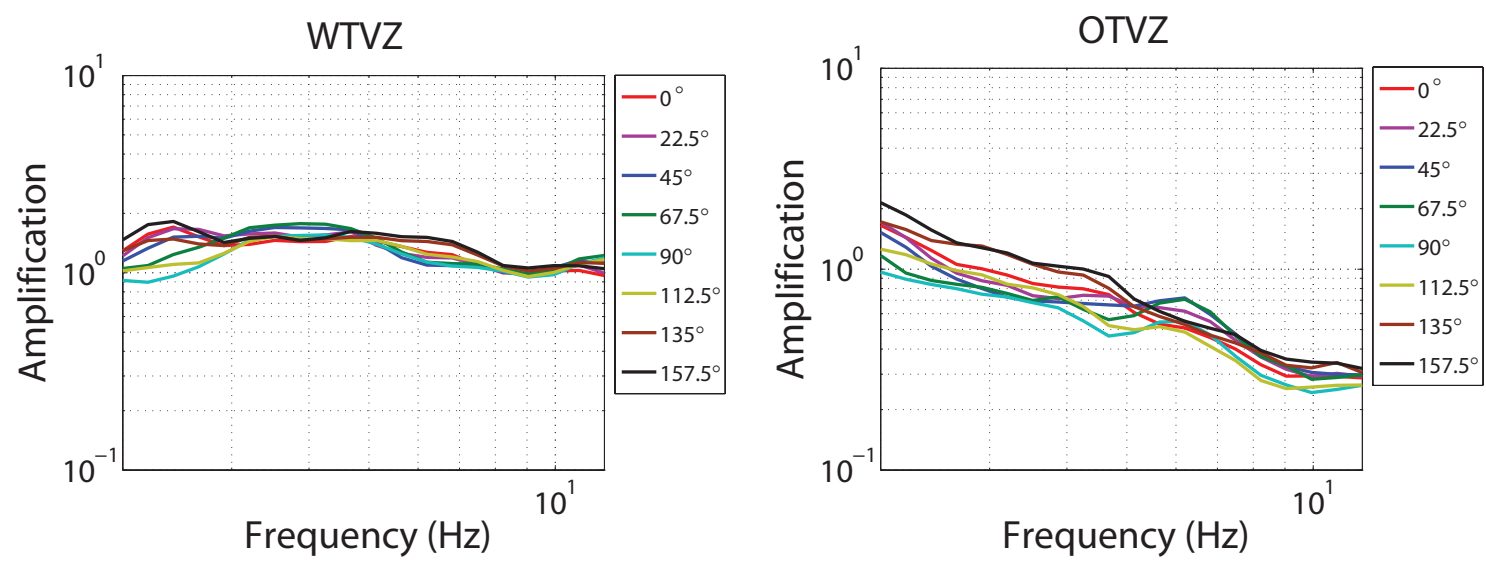

Figure 4.20 Directional site amplifications of the non-TVZ for stations WTVZ and OTVZ. 

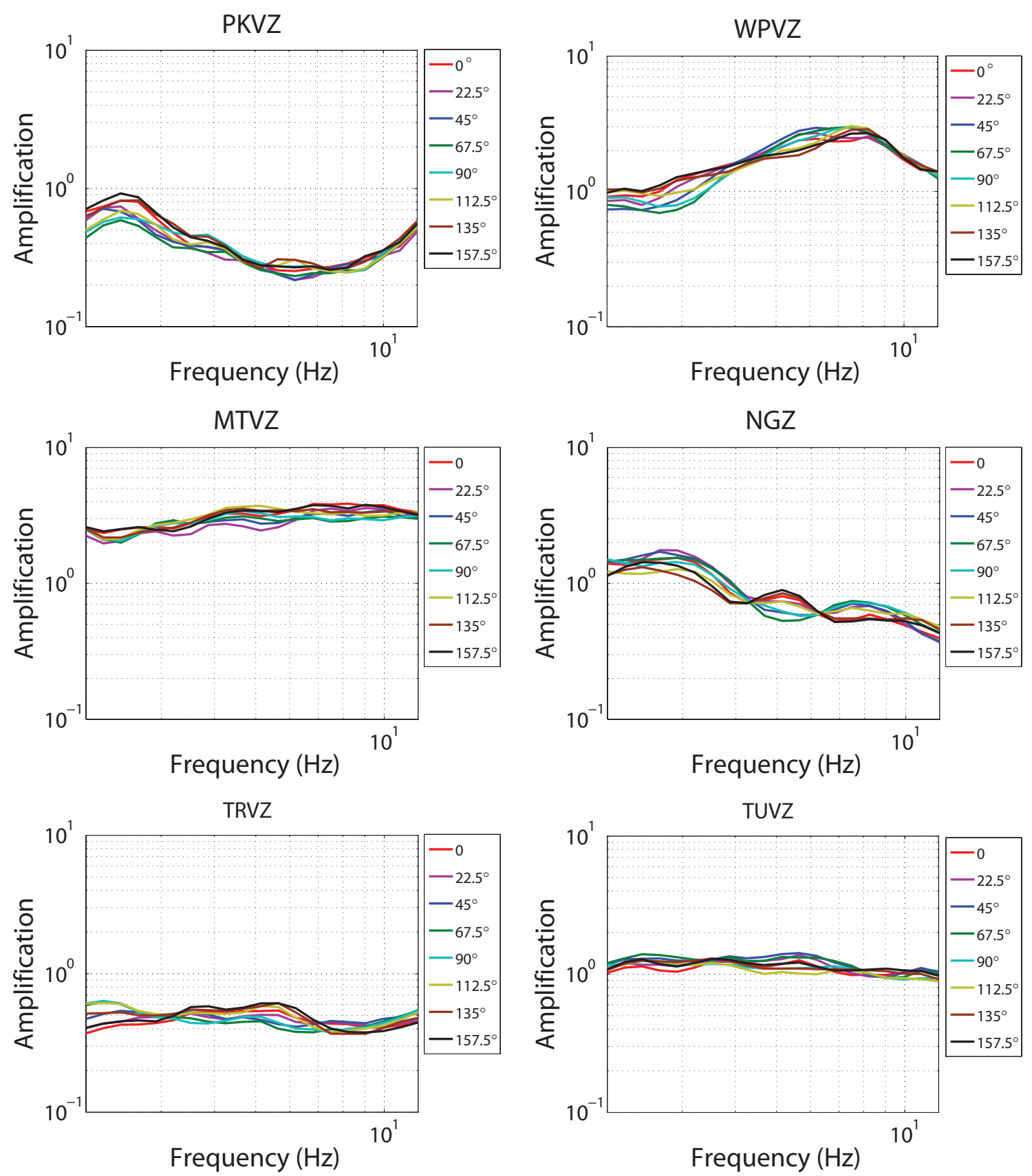

Figure 4.21 Directional site amplifications of the non-TVZ for stations PKVZ, WPVZ, MTVZ, NGZ, TRVZ and TUVZ. 

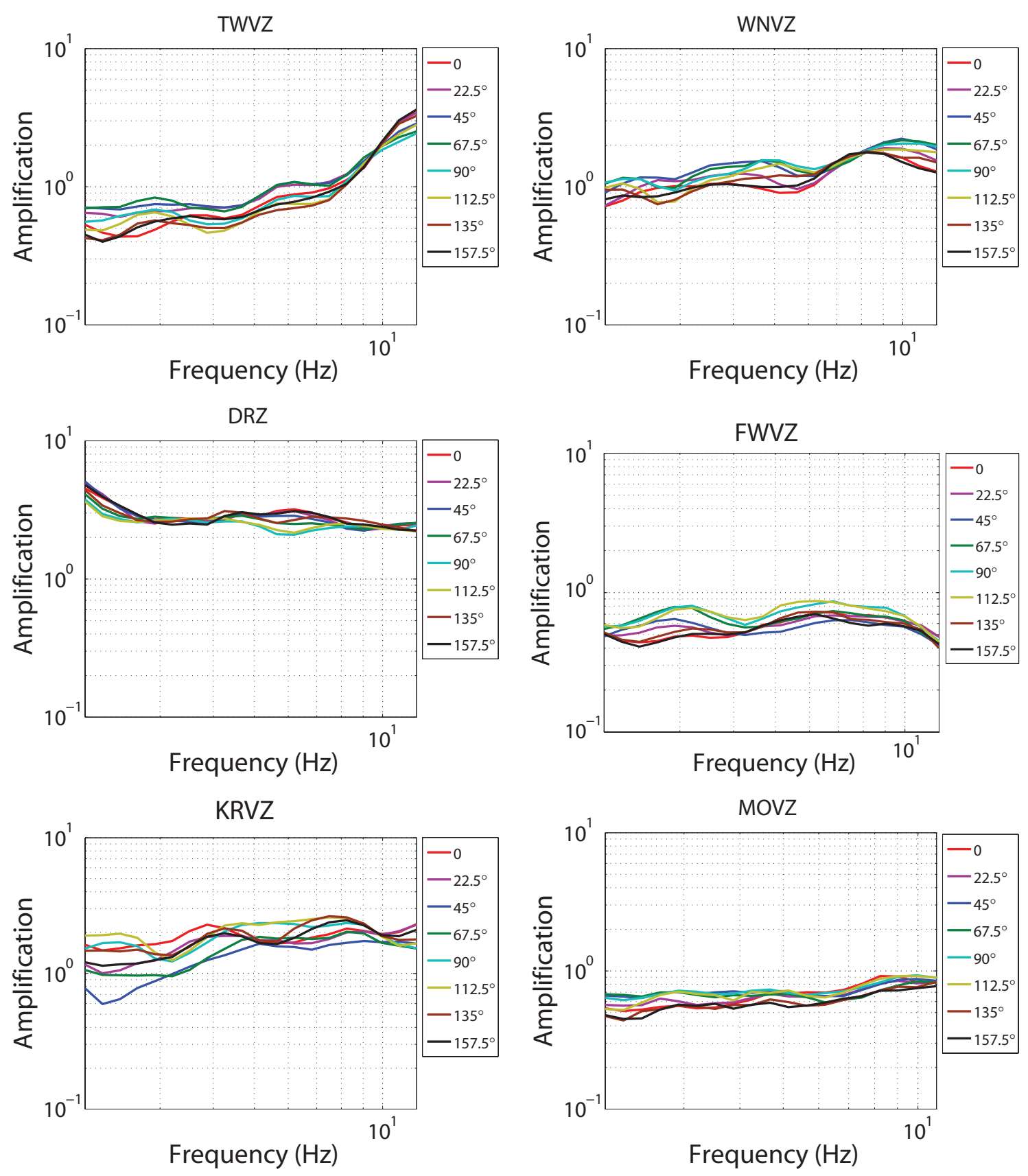

Figure 4.22 Directional site amplifications of the non-TVZ for stations TWVZ, WNVZ, DRZ, FWVZ, KRVZ and MOVZ. 


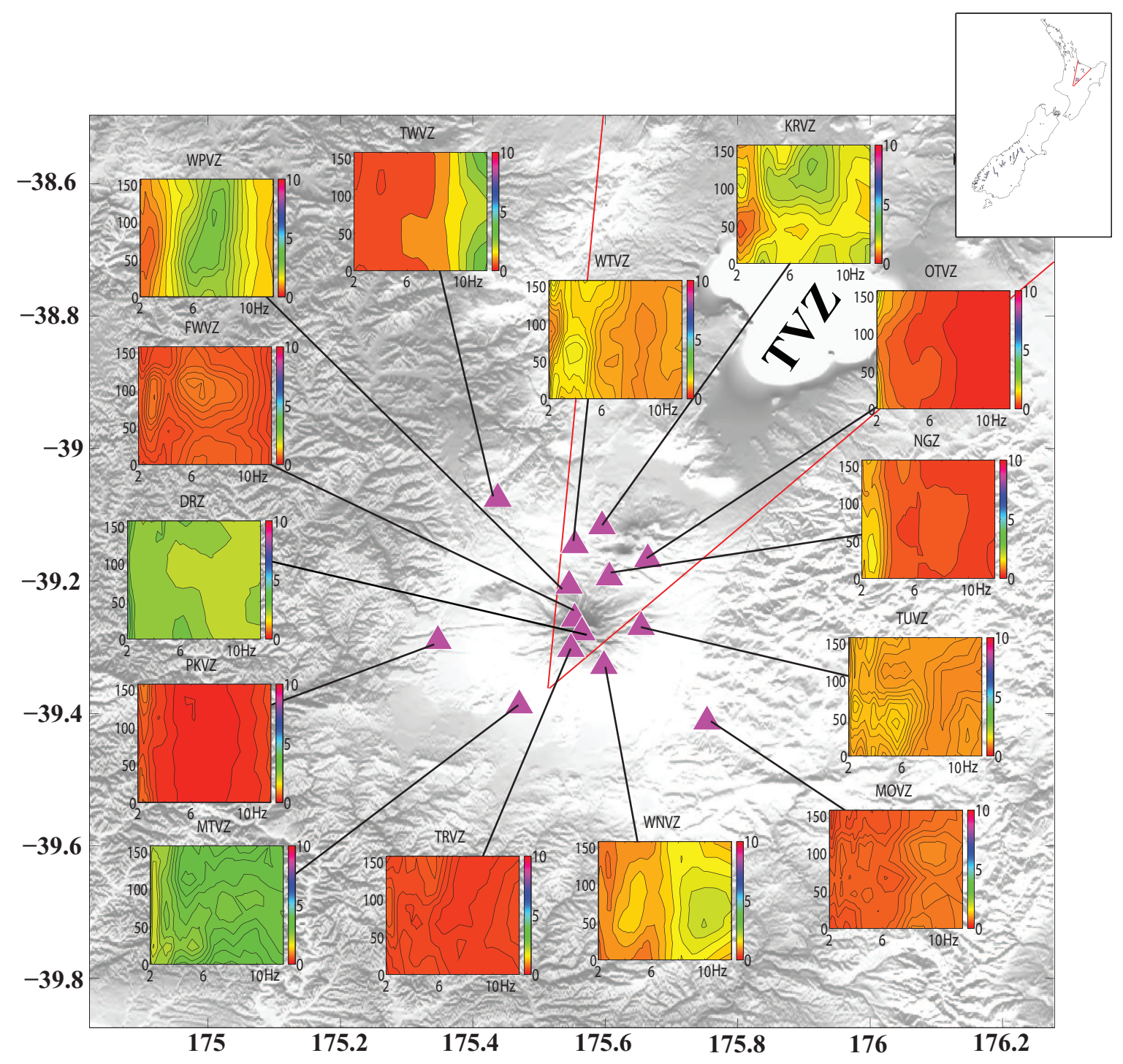

Figure 4.23 The directional site amplifications in the southernmost part of the TVZ using the non-TVZ dataset. 


\section{Chapter 5}

\section{Discussion}

We have investigated the frequency dependence of the estimated $Q_{S}$ anisotropy in the southernmost part of the TVZ by adopting the conventional frequency-dependent law in terms of $Q_{0}$ and $\alpha$ parameters (eq. 2.5) described in Chapter Two. The mechanism governing frequency-dependent seismic attenuation is still unclear, although its existence has been accepted for several decades (e.g. Aki, 1980a; Kinoshita, 1994). Aki and Chouet (1975) suggested that frequency-dependent attenuation of coda waves is due to variations in backscattering processes with depth causing strong changes in $Q$. The frequency dependence of $Q$ also has an important role to play in geophysical interpretation in terms of its association with tectonic activity. Numerous studies show that low $Q_{0}$ and high $\alpha$ are characteristic of active tectonic regions, while stable cratons are typically characterized by higher $Q_{0}$ and lower $\alpha$ (Aki, 1980b; Castro et al., 2008b,c) (see Table 2.2).

In volcanic regions, high densities of cracks or faults may increase body wave scattering, causing lower $Q$ with strong frequency dependence (e.g. Castro et al., 2008b), while the ductility associated with the presence of magma and hot temperatures may reduce the small-scale of heterogeneity, causing a dominance of intrinsic mechanism over scattering attenuation (Aki, 1980b; Giampiccolo et al., 2007). Intrinsic attenuation is characterized by a weaker frequency dependence of $Q$ (Giampiccolo et al., 2007). Thus, the frequency dependence of $Q$ can be interpreted as an indicator of the presence of hot materials and 
cracks, providing insights into the mechanisms governing attenuation overall. We consider strong frequency dependence (i.e. $\alpha>1$ (Mayeda et al., 1992)) to indicate scattering and weaker frequency dependence with $\alpha=0.2$ to 0.3 to be caused by intrinsic attenuation (Karato and Spetzler, 1990).

The geological structure of volcanic regions is commonly heterogeneous, containing fractures, cracks, dykes and other volcanic features. Such features can be oriented in preferred directions, causing the medium beneath the volcanic areas to be anisotropic. In anisotropic regions containing aligned cracks or fractures, seismic waves may propagate with different velocities and polarizations causing anisotropy in velocity: seismic waves polarised parallel in the plane of the cracks, travel faster than those polarised in a perpendicular direction. The presence of aligned cracks may also cause seismic waves to attenuate. The presence of aligned cracks or fractures may therefore establish a physical connection between seismic velocity anisotropy and attenuation anisotropy. A common crack model that has been widely used in the interpretation of seismic anisotropy is the penny-shaped crack model proposed by Hudson (1981). The theory predicts that the seismic wave propagating perpendicular to the crack's or fracture's orientation (slow shear waves) should be more highly attenuated than those which are parallel with the crack's or fracture's direction (fast shear waves).

Hudson's theory implies that the presence of aligned cracks will contribute to attenuation anisotropy. Cracks detected through attenuation anisotropy have been investigated using lab scale measurements and field observations. For example, Liu et al. (2005) found 23-30\% of attenuation anisotropy in the uppermost $200 \mathrm{~m}$ of the crust at a borehole seismometer in western part of Taiwan. They suggested that attenuation anisotropy in the near surface may be due to the contribution of microcracks' alignment, which also causes $8 \%$ velocity anisotropy at the same station. A fracturing contribution to attenuation anisotropy was also reported by Carter and Kendall (2006), who measured attenuation anisotropy using a microseismic dataset collected in an active oil-field. Interestingly, they observed that sometimes the fast waves are more attenuated than the slow waves. This finding is contra- 
dictory to Hudson's theory and it may suggest that the model is wrong. As discussed in Appendix C we initially obtained similar results when using Carter and Kendall's method. We suggest that the contradiction with the results provided using this model may be due to the directional effects of site responses as described in Section 4.4.2. When each polarization has a different amplification due to a site effect, it is possible that S-wave spectra with the slow polarization are enriched at high frequencies, while those with fast polarization are depleted, yielding a negative slope on the differential attenuation (Appendix C).

\subsection{The data}

Some of the source depths reported by GeoNet for the TVZ dataset are fixed at $5 \mathrm{~km}$ depth (Fig. 3.7). This will have caused inaccurate hypocentral distances because routine analysis probably did not determine accurately the depths of the shallow events. However, inaccurate hypocentral distances should not greatly affect the estimated attenuation functions because the basic idea of the non-parametric GIT method is to look for a smooth function of distance by suppressing rapid undulations interpreted as site effects. However, this inaccuracy may affect the measured site and source effects. For example, if the assumed hypocentral distances do differ from the true distances, they will produce amplitude variations of the attenuation function. The inversion suppresses this variation in order to obtain a smooth function. Once the attenuation function has been determined, the corrected spectra from the attenuation function are then split into source and site effects. However, the corrected data still contain amplitude variations (measurement error) resulting from the suppression in the first inversion. We conclude that the biggest effect would be in the source effect, in particular the falloff $\gamma$ of the spectral slope. The source function is not constrained in the inversion, so errors in the source may cause any shape of function to minimize residuals in a least-square sense (Akyol et al., 2002).

As already discussed in Chapter 2 and 3, the accuracy and resolution of the method depend on the density of the earthquakes used in the inversion. In our dataset, we only used 
seismograms containing S-phases picked by GeoNet. Consequently, some usable records may have been omitted from the dataset because they did not have S-phases picked during the initial analysis. In any further studies, it will be important to pick $\mathrm{S}$ waves arrival by hand to increase the raypath coverage of the dataset.

In addition, most of the earthquakes in the TVZ data have hypocentral distances less than $50 \mathrm{~km}$, even though the search criteria was to examine all earthquakes within $100 \mathrm{~km}$ of Ruapehu. This can be explained by computing the theoretical model of amplitude decay based on equation 2.4. We use an average $Q$ value ( $\sim 7$ at $1 \mathrm{~Hz}$ ) obtained for the upper crust model, because the upper crust model has $Q$ higher than the other models, allowing the Swave to propagate further. Then, we plot the theoretical model of amplitude decay showing how the S-wave energy decays with distances as shown in Figure 5.1. This figure shows that the S-wave energy decreases almost completely within $50 \mathrm{~km}$ in the TVZ, which is consistent with our dataset. In other words, the model implies that the S-waves are highly attenuated in that region.

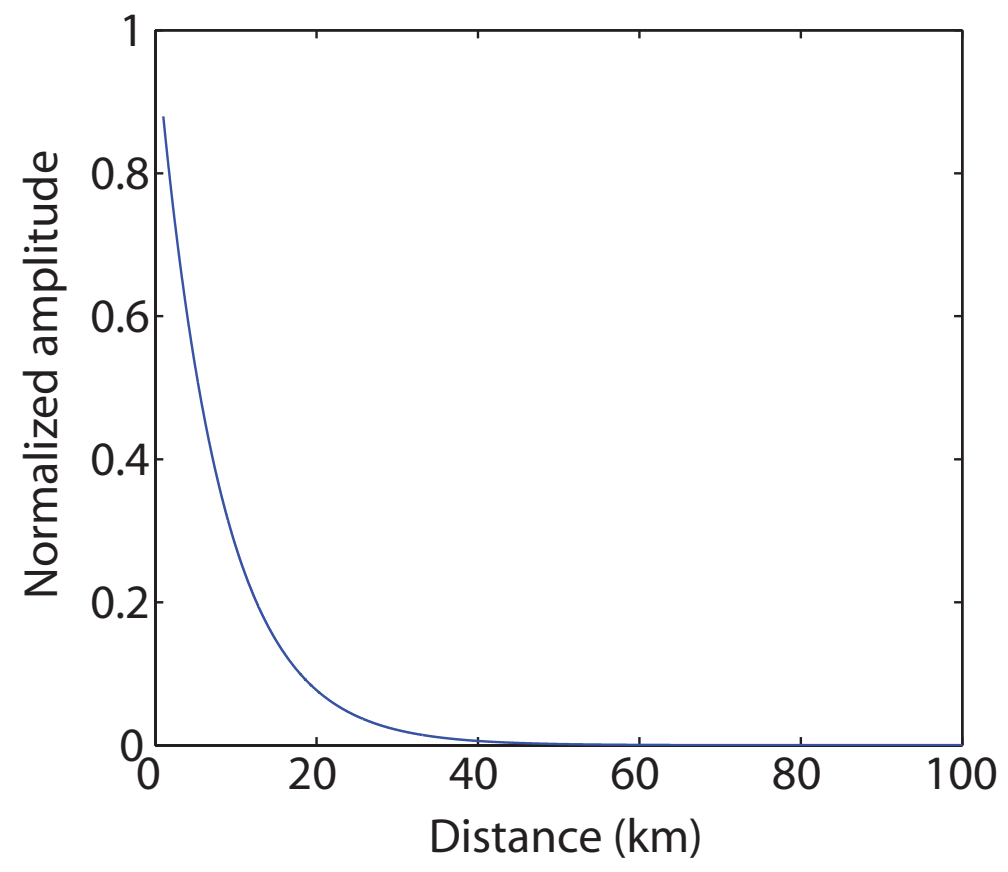

Figure 5.1 The theoretical model showing how the S-waves amplitude decay with distances. 


\subsection{Inside the TVZ}

\subsubsection{Amplitude decay functions}

In general, the amplitude decay functions obtained from $S_{E W}$ and $S_{T}$ attenuate more strongly than those obtained from $S_{N S}$ and $S_{R}$, respectively, particularly at high frequencies (Fig. 4.1 and 4.4). As high frequencies are most sensitive to scattering, we expect that this mechanism may dominate the attenuation process overall (e.g. Aki, 1980b). It is also important to note in those figures that particularly at high frequencies for both components, the functions show a change in the rate of decay for hypocentral distances greater than $25 \mathrm{~km}$, in which the attenuation function versus distance curves become more flattened.

The flattening effect apparent in the attenuation functions may reflect by several factors. Firstly, the flattening effect at $r>25 \mathrm{~km}$ may be the effect of postcritical layer reflection when the ray travels to a deeper layer of the Earth (Castro et al., 1996). An increase in velocity beneath the TVZ might cause a similar reflection; Harrison and White (2004) deduced that a $16 \mathrm{~km}$-thick quartzo-feldspathic crust is underlain to $\sim 30 \mathrm{~km}$ depth beneath the Taupo Volcanic Zone by a mafic material containing at least $2 \%$ partial melt. In contrast, Stratford and Stern (2004) reported an increase in seismic velocity for the same depth interval beneath the TVZ and suggested that the velocity structure from 16 to $30 \mathrm{~km}$ depth represents the presence of anomalously low-velocity upper mantle. Another possible factor is that of reflection due to lateral variations in crustal structure (i.e. the presence of dipping interfaces). However, the different flattening effects of the attenuation function between radial and transverse components get larger with increasing distance, which suggests that the different flattening on both components is mainly due to attenuation.

The directional attenuation-distance curves (Fig. 4.3) are probably related to the characteristic of the geological structures beneath the TVZ, such as the presence of cracks aligned in the upper crust. Such an explanation is intuitively attractive since seismic waves propagating parallel to the cracks' orientation should be less affected by scattering processes than those traveling perpendicular to the cracks. In Figure 4.3, the big difference 
between the amplitude decay rates in the E-N model, in which the decay functions obtained from the $\mathrm{N}$ component are less attenuated than those in the E component, implies the presence of cracks oriented in a N-S direction. Furthermore, the characteristic of the directional attenuation functions are consistent with a $3 D \mathrm{Vp}$ azimuthal anisotropy study in which the fast directions were found mainly to $\mathrm{N}-\mathrm{S}$ for the upper crust $(<7 \mathrm{~km})$ in the southernmost part of the TVZ (Eberhart-Phillips and Reyners, 2009).

\subsection{2 $Q$ model}

For the TVZ data, most records used in this measurement correspond to earthquakes located to the south of Lake Taupo (Fig. 3.5). Our results for the homogeneous, upper and lower crust $Q$ models show that there is a difference in the value of $Q$ between two orthogonal components (Fig. 4.6, 4.8 and 4.9) at some frequencies. These results indicate the presence of attenuation anisotropy beneath the region.

Furthermore, the TVZ data has a dominant direction for a radial component of approximately $215^{\circ}$, so we expect that the radial and transverse $Q$ model should correspond to the $Q$ model for rotations between $22.5^{\circ}$ and $45^{\circ}$. We compute an attenuation model for a rotation angle of $35^{\circ}$ to test the consistency of the radial and transverse model.
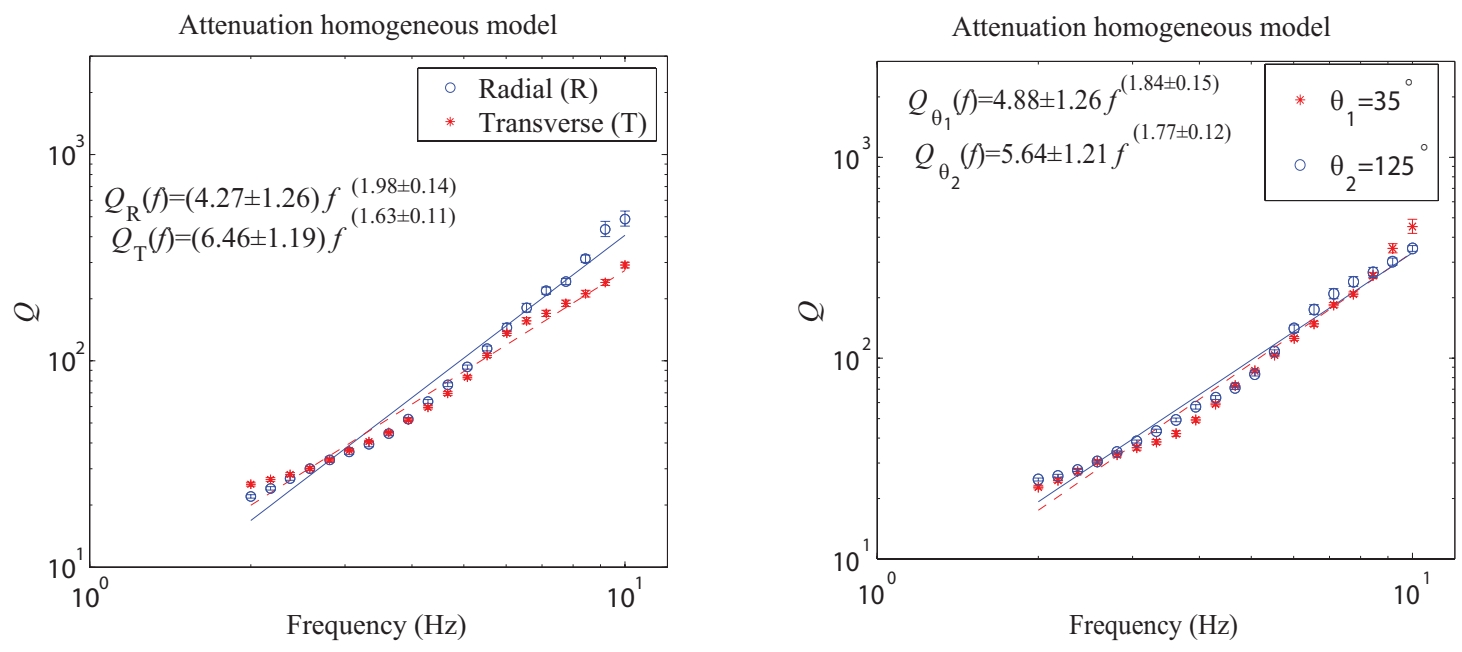

Figure 5.2 Comparison $Q$ values to test the consistency of the radial and transverse model. 
Figure 5.2 shows that there is no appreciable difference between $Q$ values of the two orthogonal components on the $35^{\circ}$ models, while the $\mathrm{R}$ and $\mathrm{T}$ model shows that the $Q$ values of the transverse component are lower than those of radial component at high frequencies. A plausible explanation regarding the discrepancy is that the waves on the radial component travel along the raypath from the source to the stations in the vertical plane, while those on the transverse component propagate in the horizontal plane. Thus, the existence of vertical cracks may cause the waves on the transverse component $(\mathrm{SH})$ to be more attenuated than those on the radial component. The previous discussion suggests that the orientation of the vertical cracks is $\mathrm{N}-\mathrm{S}$, at angle of $35^{\circ}$ to the dominant radial direction.

\section{Homogeneous $Q$ model}

In general, the low values of homogeneous $Q$ (Fig. 4.6) obtained for S-waves beneath the TVZ agree with those found in other volcanic regions. For example, Castro et al. (2008b) found a strong frequency dependence of $Q$ described by the relation $Q_{S}=17.8 f^{1.3}$ for a highly volcanically active region in the southern part of Italy. Styles (2009) found low S-wave $Q_{S}$ of $\sim 350$ at a frequency of $10 \mathrm{~Hz}$ and depths shallower than $15 \mathrm{~km}$ in the southernmost part of the TVZ. She determined $Q_{S}$ at $10 \mathrm{~Hz}$ and extrapolated to other frequencies by assuming $\alpha=0.3$. By using this power law for the frequency dependence of $Q$, we can compare our results with Styles' average $Q$ results (Fig. 5.3). We take an average value of $Q_{0}=5$ and $\alpha=1.8$ for our data.

Figure 5.3 shows that our results are comparable with the average values obtained from Styles' $Q_{S}$ tomography study at $f=10 \mathrm{~Hz}$. At frequencies less than $10 \mathrm{~Hz}$, our results imply lower $Q$ than Styles' results. This can be explained because Styles' study mainly focused on the attenuation structure in the mantle and subduction zone, so she assumed $\alpha=0.3$ (Karato and Spetzler (1990) suggest $\alpha$ values ranging $0.1-0.3$ for mantle rocks), instead of the value of $\alpha>1$ that we determined for the crust in the volcanic region.

The homogeneous $Q$ model also shows that the pair of orthogonal seismograms in the $\mathrm{N}-\mathrm{S}$ and $\mathrm{E}-\mathrm{W}$ directions yield maximum attenuation anisotropy, in which S-waves 


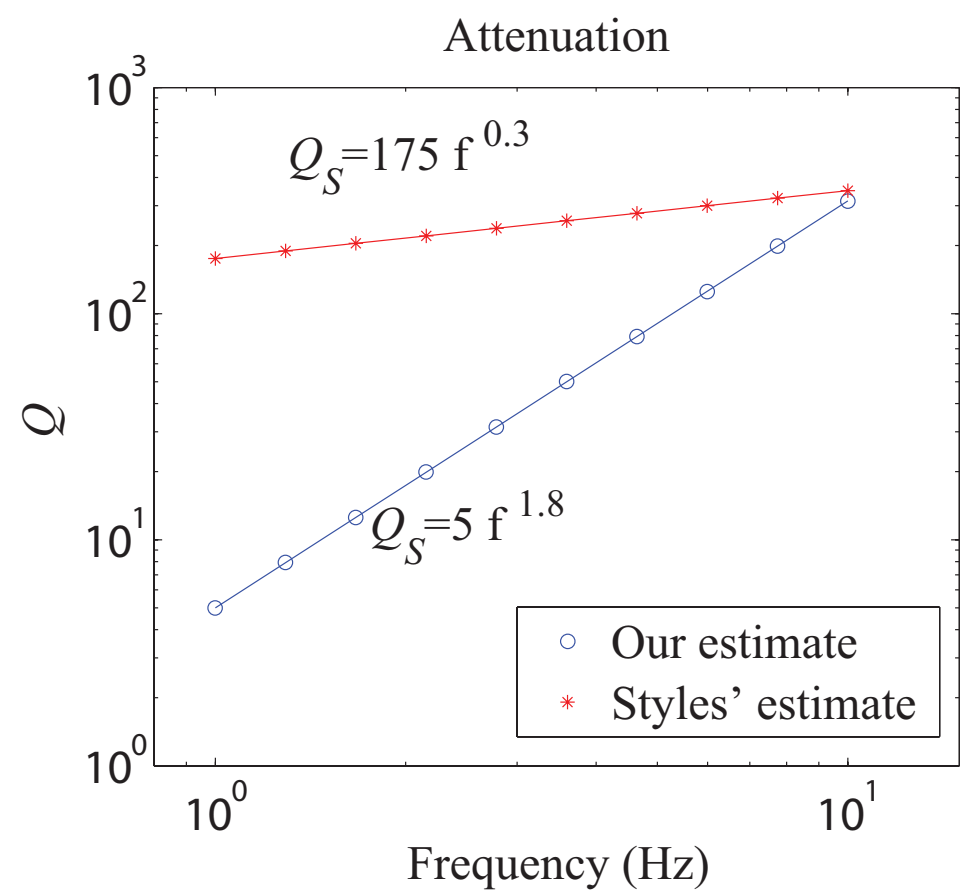

Figure 5.3 Comparison of average $Q$ values obtained in this and Styles'(2009) studies.

polarised in the $\mathrm{N}-\mathrm{S}$ direction are less attenuated than those polarised in the $\mathrm{E}-\mathrm{W}$ orientation. The attenuation anisotropy implied by this model exists mainly at high frequencies ( $\mathrm{f}>6 \mathrm{~Hz}$ ), where we expect that scattering due to crack alignment provides the dominant contribution. Adopting the average S-wave velocity of $3.2 \mathrm{~km} / \mathrm{s}$ used in the homogenous $Q$ model for the 6-10 Hz frequency band of the N-E $Q$ model (Fig. 4.6), the wavelength of the anisotropic S-waves is computed to be of the order of 300 to $500 \mathrm{~m}$. This range may be comparable to the dimensions of dikes and other volcanic features, and we infer on the basis of the homogeneous model that the attenuation anisotropy beneath the TVZ may be caused by scattering due to heterogeneities.

\section{Two-layer $Q$ model}

The simple single layer $Q$ model does not distinguish the contributions of the upper and lower crust to the observed attenuation anisotropy. We anticipate waves traveling in the upper crust to be sensitive to cracks' orientations while those propagating in the lower crust 
beneath the TVZ to be affected by the presence of mineral fabric or partial melt, providing information about their influence on attenuation. With this motivation, we also considered a two-layer model (Section 4.2.2) for comparison with the homogeneous model.

The results shown in Figure 4.8 imply that the maximum attenuation anisotropy in the upper crust occurs at high frequencies $(f>7 \mathrm{~Hz})$ in the $\mathrm{E}-\mathrm{N}$ geometry. The slight difference between the statistical uncertainties of the estimates indicates a weak attenuation anisotropy in the upper crust. However, the small difference between the $Q_{R}$ and $Q_{T}$ estimates is within the statistical uncertainties of the measurements.

The N-S direction of lower attenuation for the upper crust south of Lake Taupo is comparable to the $\mathrm{N}-\mathrm{S}$ fast orientation of seismic anisotropy obtained using inversion of 3D travel time data (Eberhart-Phillips and Reyners, 2009), as already discussed in Section 5.2.1. The smaller values of anisotropy in this model compared to the homogeneous model may reflect heterogeneous structure as reported also by Eberhart-Phillips and Reyners (2009) and Styles (2009).

For the deeper portion of the crust ( $>9 \mathrm{~km}$ ), the $Q$ values tend to be lower than those obtained for the upper crust (Fig. 4.8 and 4.9). The lower crust results show that the principal orientation of attenuation anisotropy is radial, with $Q_{R}$ greater than $Q_{T}$ at high frequencies (f $>5 \mathrm{~Hz}$ ) (Figure 4.9). Conversely, in the azimuthal plane, $Q$ values in the $45^{\circ}$ direction have higher attenuation than those in the orthogonal direction at most frequencies.

Although the $Q$ values reveal a strong overall frequency dependence, suggesting that scattering may still dominate the attenuation mechanism, the frequency dependence is less pronounced at some frequencies (e.g. 7-10 Hz), suggesting a contribution of intrinsic attenuation. Thus, the attenuation anisotropy for depths greater than $9 \mathrm{~km}$ in this region may not be related to spatially extensive fracturing associated with the TVZ's ongoing extension, because extensive fracturing should be oriented $45^{\circ}$ causing low attenuation in that direction. Our results are consistent with the higher $Q$ direction coinciding with the fast velocity direction obtained from the 3D Vp azimuthal anisotropy 
study (Eberhart-Phillips and Reyners, 2009). Eberhart-Phillips and Reyners (2009) observed that the fast direction within the TVZ in the lower crust has a northwest orientation, parallel to the extension direction of the TVZ. They argued this could be related to extensional strain features in a ductile material, since the lower crust in the TVZ would be ductile as indicated by the shallow seismogenic zone (Reyners et al., 2006). However, attenuation anisotropy in ductile materials is not well understood: we know of no geophysical observations or theoretical models addressing the contribution of ductile materials to attenuation anisotropy. Further studies are necessary to reveal the exact mechanism of attenuation anisotropy in ductile materials, such as those that likely form the lower crust beneath the TVZ.

Attenuation anisotropy accompanying seismic velocity anisotropy in the lower crust has been also reported in another region by Castro et al. (1999). Castro et al. measured attenuation anisotropy in the Marche region of Italy, providing additional evidence of seismic anisotropy reported by previous seismic velocity anisotropy studies (e.g. Margheriti et al., 1996). Castro et al. observed low attenuation for the radial component compared to the transverse component at low frequencies ( $<4 \mathrm{~Hz}$ ), which they interpreted as due to magmatic activity related to the compressional tectonics in the region of Marche.

\subsection{Outside the TVZ}

$Q_{S}$ determined from the non-TVZ dataset is higher than that obtained from the TVZ dataset. The frequency dependence obtained for all seismogram directions is also very weak and nearly frequency-independent at frequencies of $2-7 \mathrm{~Hz}$, with maximum attenuation anisotropy occuring in the NE-SW direction $\left(45^{\circ}\right)$ at frequencies less than $7 \mathrm{~Hz}$ (Fig. 4.12). In contrast, attenuation anisotropy for the TVZ data mainly occurs at high frequencies $(f>6 \mathrm{~Hz})$ and exhibits strong frequency dependence. These results imply that the two regions have different attenuation anisotropy mechanisms.

$Q$ values with weak frequency dependence obtained for the non-TVZ data indicate that 
intrinsic attenuation dominates over scattering, in contrast to the scattering inferred for the TVZ on the basis of the pronounced frequency-dependence. The different attenuation mechanisms inferred to be characteristic of the two regions could be an artefact related to the depths of earthquakes used in the datasets (Fig. 3.9 and 3.7). The non-TVZ data have generally deeper focal depths $(>10 \mathrm{~km}$ ) than the TVZ (mostly less than $10 \mathrm{~km}$ depth) causing the waves to penetrate deeper into the Earth. Some studies show that the domination of intrinsic mechanism over scattering increases with depth (e.g. Aki, 1980b; Martynov et al., 1999; Castro et al., 2009). We expect that the contribution of scattering to the attenuation anisotropy would be significant for the TVZ due to shallower ray paths used in the dataset and heterogeneities within the volcanic area.

As noted above, most of the records in the non-TVZ dataset have focal depths greater than $10 \mathrm{~km}$ (Fig. 3.9). They are also located in the boundary of the southern termination of the TVZ in the vicinity of what is known as the Taranaki-Ruapehu line (TRL) (Fig. 3.5). The region north of the TRL has thinned crust $(20-25 \mathrm{~km})$, while south of the TRL has thickened crust ( $\sim 40 \mathrm{~km}$ ) (Stern and Davey, 1987; Reyners et al., 2007; Stern et al., 2010). A combined seismic attenuation and magnetotellurics study (Salmon, 2008) has revealed that the crust in the region south of the Taranaki-Ruapehu line is underlain by cool highdensity lithospheric mantle $\left(650^{\circ} \mathrm{C}\right)$ with high resistivity $(>1000 \Omega \mathrm{m})$ and low attenuation $\left(Q_{p}>1000\right)$. The higher $Q_{S}$ obtained in this study suggests that the non-TVZ waves travel through this structure. In addition, the effect of thickened crust also appears on the attenuation function, in which the decay curves are a bit steeper at distances greater than $35 \mathrm{~km}$ (Fig. 4.11).

\subsection{Source and site effects}

\section{Source effect}

We estimated the source parameters of regional earthquakes around the TVZ in terms of the low-frequency amplitude level $\left(\Omega_{0}\right)$, the high frequency fall-off $(\gamma)$ and the corner 
frequency $\left(f_{c}\right)$ using the source effects obtained in the second step inversion of the nonparametric method (Section 3.1.1). The source effect can be separated from the site effect by imposing the constraint that the average site effect equals one. The constraint is also applied in the first step of the inversion when calculating $Q$. We constrained the attenuation functions to be smooth functions of distance. This constraint is needed to avoid strong trade-offs or ambiguities causing large errors during inversion. In the second step of the inversion, however, both the source and site effects depend on frequency, meaning that any tradeoff or ambiguity cannot be reduced completely (Castro et al., 1990). Furthermore, the source function is not constrained in the inversion. This implies that the source function may have any functional shape in order to reduce residuals in a least-squares sense (Akyol et al., 2002).

The slope of the source spectrum at high frequencies, described by the parameter $\gamma$, has values ranging from 1.9 to 4.9. For clarity, we rewrite Boatwright's equation as

$$
S(f)=\Omega_{0} /\left[1+\left(f / f_{c}\right)^{2 \gamma}\right]^{1 / 2}
$$

These results suggest that the events decay at rates proportional to approximately $\omega^{-2}$ and $\omega^{-3}$ or greater. Most of the simple theoretical models yield $\gamma$ of about 2-3 (Ambeh and Fairhead, 1991). For instance, Brune (1970) found an $\omega^{-2}$ decay model, which is equivalent to $\gamma=2$. However, the fact that the high frequency fall-off $(\gamma)$ has values greater than 3, may imply either the presence of some attenuation mechanism other than path effects as defined on equation 3.6 or complicated source functions which cannot be defined as simple theoretical source models such as Brune's source model (Ambeh and Fairhead, 1991). Another possible source of $\gamma$ greater than 3 is that the TVZ dataset are fixed at $5 \mathrm{~km}$ depth affecting the source functions in the inversion as described in Section 5.1.

It is difficult to compare the corner frequencies $\left(f_{c}\right)$ obtained in this study and by Styles (2009), because we use different earthquakes and the corner frequency values may vary for different earthquakes depending on source size and rupture complexity (Stein and Wysession, 2003). However, our $f_{c}$ values, which range from 2.2 to $4.9 \mathrm{~Hz}$, are generally comparable to those obtained from the 3D S-wave tomography study (Styles, 2009). Styles 
(2009) obtained $f_{c}$ values ranging from about 3.7 to $13.8 \mathrm{~Hz}$ by assuming that the high frequency spectra of the earthquake decay was $\omega^{-2}$ following Brune's source model. Further analysis is needed to determine other source parameters describing earthquakes scaling relationships and the stress drop of local earthquakes in the central North Island.

\section{Site effects}

The results from two different groups of source locations (Fig. 3.5) show that the amplifications at the GeoNet sites are approximately the same for both datasets, as expected if the site response is independent of earthquake location. However, the amplifications at stations PKVZ, MTVZ and DRZ for the TVZ data are higher than those for the non-TVZ data (Fig. 4.15- 4.16 and Fig. 4.21-4.22). A possible explanation is that a directional effect may exist in this region. All the earthquakes in the non-TVZ data are located closer to the stations and deeper than those within the TVZ, causing the rays to travel nearly vertically to the stations (Fig. 3.5). As a consequence, the TVZ and non-TVZ data are expected to have different incident wavefields. In this case, we suggest that as the angle of incidence increases, the amplification decreases (e.g. Frischknecht and Wagner, 2004). Another possibility is that could be due to lack of TVZ earthquakes observed by those stations (Table 4.6). This causes the site amplification of the three stations to be poorly constrained. Additional work is needed to study the intriguing possibility of directional site functions in this region. 


\section{Chapter 6}

\section{Summary}

We have analyzed earthquake data sets from two different regions in the southernmost part of the TVZ with the aim of studying the mechanisms governing attenuation anisotropy. Our results reveal the presence of attenuation anisotropy in the southernmost part of the TVZ. We find a connection between the frequency dependence of S-wave attenuation anisotropy and geological features beneath the region: in particular, in the upper and lower crust. The findings are consistent with a general theoretical framework (Hudson, 1981) and with the results of previous structural and seismological investigations in the southernmost part of the TVZ (e.g. Styles, 2009; Eberhart-Phillips and Reyners, 2009).

The low values of $Q_{S}$ exhibiting strong frequency dependence obtained within the TVZ are comparable to those found in other volcanic regions (e.g. Castro et al., 2008b). The frequency dependence of $Q_{S}$ beneath this region suggests that scattering may dominate over intrinsic attenuation, reflecting heterogeneities within the volcanic area. If we consider the variation of $Q$ with depth, the weak attenuation anisotropy in the N$\mathrm{S}$ direction found for the upper crust can be related to geological heterogeneity (e.g. Eberhart-Phillips and Reyners, 2009; Styles, 2009). Conversely attenuation anisotropy in the lower part of the crust beneath the TVZ is aligned in a northwest orientation and consis-

tent with results of the 3D Vp azimuthal anisotropy study (Eberhart-Phillips and Reyners, 2009), which those authors related to extensional strain features in ductile materials. 
The $Q_{S}$ values obtained from the non-TVZ dataset indicate that $\mathrm{S}$-waves are less attenuated outside the TVZ than those within the TVZ. The weak frequency-dependence of $Q_{S}$ observed at low frequencies outside the TVZ suggests the dominance of intrinsic attenuation over scattering. Furthermore, attenuation anisotropy mainly occurs at low frequencies for the non-TVZ and at high frequencies for the TVZ, indicating that attenuation in the two regions reflects different attenuation anisotropy mechanisms.

The source parameters of the earthquakes analyzed for the TVZ, in particular the high frequency fall-off $(\gamma)$ and the corner frequency $\left(f_{c}\right)$, indicate that the spectra decay at close to $\omega^{-2}$ and $\omega^{-3}$ or greater. However, the fact that the high frequency fall-off $(\gamma)>2$ and attenuation frequency dependence coefficient $(\alpha)>1$ as mentioned in Chapter 4 and 5 may result as a trade-off between the source parameters and the attenuation terms. The high $\alpha$ seems contrary to the fact that the high frequencies of body waves are usually attenuated more rapidly than that of lower frequencies in the highly attenuating regions (Lowrie, 1999). Furthermore, the trade-off may also lead to an ambiguity of the seismic moment $(\Omega)$ results (Chapter 4 ) which should be the same for the two different components of the seismograms. It is expected that there may be correlation between the errors in $\alpha$ and $\gamma$. Therefore, further research is needed to reveal the possible trade-off.

The site amplification results as a function of polarization angles and source locations are generally similar for both datasets, suggesting that the observed site functions may be independent of the earthquakes' locations. A few stations show an indication of directional site effects, although this observation requires further analysis. Further investigation is necessary in order to substantiate the possibility of directional site effects around the TVZ. 


\section{References}

Aki, K. (1980a). Attenuation of shear waves in the lithosphere for frequencies from 0.05 to $25 \mathrm{~Hz}$. Phys Earth Planet In, 21.

Aki, K. (1980b). Scattering and attenuation of shear waves in the lithosphere. J. Geophys. Res., 85:6496-6504.

Aki, K. and Chouet, B. (1975). Origin of coda waves: Source, attenuation and scattering effects. J. Geophys. Res., 80:3322-3342.

Aki, K. and Richards, P. G. (1980). Quantitative Seismology. University Science Books, 2nd edition.

Akyol, N., Akinci, A., and Eydogan, H. (2002). Separation of source, propagation and site effects from $\mathrm{S}$ waves of local earthquakes in Bursa region, northwestern Turkey. Pure Appl. Geophys., 159:1253-1269.

Ambeh, W. B. and Fairhead, J. D. (1991). Spectral characteristics and source parameters of microearthquakes from the Mt Cameroon volcanic region, West Africa. Geophys. J. Int., 106:229-237.

Anderson, J. G. and Hough, S. E. (1984). A model for the shape of the fourier amplitude spectrum of acceleration at high frequencies. Bull. Seism. Soc. Am., 74:1969-1993.

Andrews, D. J. (1986). Objective determination of source parameters and similarity of earthquakes of different sizes. In Boatwright, J., Scholz, C. H., and Das, S., editors, 
Earthquake Source Mechanic, American Geophysical Union, pages 259-267, Washington, DC.

Ansell, J. and Bannister, S. (1996). Shallow morphology of the subducted Pacific plate along the Hikurangi margin, New Zealand. Phys. Earth. Planet. Inter, 93:3-20.

Arevalo, C. M., Bianco, F., Ibanez, J. M., and Del pezzo, E. (2003). Shallow seismic attenuation and shear wave splitting in the short period range of Deception Island volcano (Antarctica). J. Volcanol. Geoth. Res., 128:89-113.

Bannister, S., Bryan, C. J., and Bibby, H. M. (2004). Shear wave velocity variation across the Taupo Volcanic Zone, New Zealand, from receiver function inversion. Geophys. J. Int., 159:291-310.

Barton, N. (2007). Rock quality, seismic velocity, attenuation and anisotropy. Taylor and Francis Group, London.

Bath, M. (1974). Spectral analysis in geophysics (Development in Solid Earth Geophysics). Elsevier, Amsterdam.

Beavan, J., Tregoning, P., Bevis, M., Kato, T., and Maeertens, C. (2002). Motion and rigidity of the Pacific Plate and implications for plate boundary deformation. J. Geoph. Res., 107:2261.

Bibby, H. M., Caldwell, T. G., Davey, F. J., and Webb, T. H. (1995). Geophysical evidence on the structure of the Taupo Volcanic Zone and its hydrothermal circulation. $J$. Volcanol. Geoth. Res., 68:29-58.

Boatwright, J. (1978). Detailed analysis of two small New York State earthquake sequence. Bull. Seism. Soc. Am., 68:1117-1131.

Brune, J. N. (1970). Tectonic stress and the spectra of seismic shear waves from earthquakes. J. Geophys. Res., 75:4997-5009. 
Bryan, C. J. and Sherburn, S. (1999). Seismicity associated with the 1995-1996 eruptions of Ruapehu volcano, New Zealand : narrative and insights into physical processes. $J$. Volcanol. Geoth. Res., 90:1-18.

Carter, A. J. and Kendall, J. M. (2006). Attenuation anisotropy and the relative frequency content of split shear-wave. Geophys. J. Int., 165:865-874.

Castro, R. R., Anderson, J. G., and Singh, S. K. (1990). Site response, attenuation and source spectra of $\mathrm{S}$ waves along the Guerrero, Mexico, Subduction zone. Bull. Seism. Soc. Am., 80:1481-1503.

Castro, R. R., Condori, C., Romero, O., Jacques, C., and Suter, M. (2008a). Seismic attenuation in northeastern Sonora,Mexico. Bull. Seism. Soc. Am., 98:722-732.

Castro, R. R., Fabriol, H., Bour, M., and Le Brun, B. (2003). Attenuation and site effects in the region of Guadeloupe, Lesser Antilles. Bull. Seism. Soc. Am., 93:612-626.

Castro, R. R., Gallipoli, M. R., and Mucciarelli, M. (2008b). Crustal Q in southern Italy determined from regional earthquakes. Tectonophysics, 457:96-101.

Castro, R. R., Huerta, C. I., Romero, O., Jacques, C., Hurtado, A., and Fernndez, A. I. (2009). Body-wave attenuation near the rupture of the 1887 Sonora, Mexico, earthquake (MW 7.5). Geofsica Internacional, 48:297-304.

Castro, R. R., Massa, M., Augliera, P., and Pacor, F. (2008c). Body-wave attenuation in the region of Garda, Italy. Pure appl. geophys, 165:1351-1366.

Castro, R. R., Monachesi, G., Mucciarelli, M., Trojani, L., and Pacor, F. (1999). P- and S-wave attenuation in the region of Marche, Italy. Tectonophysics, 302:123-132.

Castro, R. R., Pacor, F., Sala, A., and Petrungaro, C. (1996). S wave attenuation and site effects in the region of Friuli, Italy. Bull. Seism. Soc. Am., 101:22355-22369. 
Castro, R. R., Rebollar, C. J., Inzunza, L., Orozco, L., Sanchez, J., Galvez, O., Farfan, F. J., and Mendez, I. (1997). Direct body-wave Q estimates in northern Baja California, Mexico. Phys Earth Planet In., 103:33-38.

Cole, J. (1990). Structural control and origin of volcanism in the Taupo volcanic zone, New Zealand. Bull. Volcanol., 52:445-459.

Del Pezzo, E., Bianco, F., and Zaccarelli, L. (2006). Separation of Qi and Qs from passive data at Mt. Vesuvius: A reappraisal of the seismic attenuation estimates. Phys Earth Planet In., 159:202-212.

Del Pezzo, E., De Natale, G., Scarcella, G., and Zollo, A. (1985). $q_{c}$ of the three component seismograms of volcanic microearthquakes at Campi Flegrei Volcanic area-Southern Italy. Pure Appl. Geophys, 123:683-696.

Donoghue, S. L., Neall, V. E., and Palmer, A. S. (1995). Stratigraphy and chronology of late Quaternary andesitic tephra deposits, Tongariro Volcanic Centre, New Zealand. Journal of the Royal Society of New Zealand, pages 115-206.

Dutta, U., Martirosyan, A., Biswas, N., Papageorgiou, A., and Combellick, R. (2001). Estimation of S-Wave site response in Anchorage, Alaska, from weak-motion data using Generalized Inversion Method. Bull. Seism. Soc. Am., 91:335-346.

Eberhart-Phillips, D. and Reyners, M. (2009). Three-dimensional distribution of seismic anisotropy in the Hikurangi subduction zone beneath the central North Island, New Zealand. J. Geophys. Res., 114:B06301.

Eberhart-Phillips, D., Reyners, M., Chadwick, M., and Stuart, G. (2008). Threedimensional attenuation structure of the Hikurangi subduction zone in the central North Island, New Zealand. Geophys. J. Int., 174:418-434.

Efron, B. (1979). Bootstrap methods, another look at the jackknife. Ann. Stat., 7:1-26. 
Feustel, A. J. (1998). Seismic attenuation in underground mines: a comparative evaluation of methods and results. Tectonophysics, 289:31-49.

Field, E. H. and Jacob, K. H. (1995). A comparison and test of various site response estimation techniques, including three that are non reference-site dependent. Bull. Seism. Soc. Am., 85:1127-1143.

Frischknecht, C. and Wagner, J.-J. (2004). Seismic soil effects in an embanked deep alpine valley: a numerical investigation of 2-D resonance. Bull. seism. Soc. Am., 94:171-186.

Gamble, J. A., Price, R. C., Smith, I. E. M., McIntosh, W. C., and Dunbar, N. W. (2003). ${ }^{40} \mathrm{Ar} /{ }^{39} \mathrm{Ar}$ geochronology of magmatic activity, magma flux and hazards at Ruapehu volcano, Taupo Volcanic Zone, New Zealand. J. Volcanol. Geoth. Res., 120:271-287.

Gerst, A. and Savage, M. (2004). Seismic anisotropy beneath Ruapehu Volcano: a possible eruption forecasting tool. Science, 306:1543-1547.

Giampiccolo, E., D’Amico, S., Patane, D., and Gresta, S. (2007). Attenuation of source parameters of shallow microearthquakes at Mt. Etna volcano, Italy. Bull. Seism. Soc. Am., 97:184-197.

Gudmundsson, O., Finlayson, D. M., Itikarai, I., Nishimura, Y., and Johnson, W. R. (2004). Seismic attenuation at Rabaul volcano, Papua New Guinea. J. Volcanol. Geoth. Res., 130:77-92.

Hackett, W. R. (1985). Geology and petrology of Ruapehu volcano and related vents. $\mathrm{PhD}$ thesis, School of Geography, Enviromental and Earth Sciences, Victoria University of Wellington, Wellington, New Zealand.

Harrison, A. J. and White, R. S. (2004). Crustal structure of the Taupo Volcanic Zone, New Zealand: Stretching and igneous intrusion. Geophys. Res. Lett., 31:L13615.

Hartzell, S. (1992). Site response estimation from earthquake data. Bull. Seism. Soc. Am., $82: 2308-2327$. 
Houghton, B. F., Wilson, C. J. N., McWilliams, M., Lanphere, M. A., Weaver, S. D., Briggs, R. M., and Pringle, M. S. (1995). Chronology and dynamics of a large silicic magmatic system: central Taupo Volcanic Zone, New Zealand. Geology, 23:13-16.

Hudson, J. A. (1981). Wave speeds and attenuation of elastic waves in material containing cracks. Geophys. J. Roy. Astron. Soc., 64:133-150.

Karato, S. and Spetzler, H. A. (1990). Defect microdynamics in minerals and solid-state mechanisms of seismic wave attenuation and velocity dispersion in the mantle. Reviews of Geophysics, 28:399-421.

Kinoshita, S. (1994). Frequency-dependent attenuation of shear waves in the crust of the southern Kanto area, Japan. Bull. Seism. Soc. Am., 84:1387-1396.

Konno, K. and Omachi, T. (1998). Ground-motion characteristics estimated from spectral ratio between horizontal and vertical components of microtremors. Bull. Seism. Soc. Am., 88:1228-1241.

Lamarche, G. and Lebrun, J. F. (2000). Transition from strike-slip faulting to oblique subduction: active tectonics at the Puysegur Margin, South New Zealand. Tectonophysics, 316:67-89.

Liu, Y., Teng, T., and Ben-Zion, Y. (2005). Near-surface seismic anisotropy, attenuation and dispersion in the aftershock region of the 1999 Chi-Chi earthquake. Geophys. J. Int., 160:695-706.

Lowrie, W. (1999). Fundamentals of Geophysics. Cambridge University Press.

Luetgert, J. H. (1992). Interactive two-dimensional seismic raytracing for the Macintosh. U.S. Geol. Surv., Open File Report, pages 92-356.

Margheriti, L., Nostro, C., Cocco, M., and Amato, A. (1996). Seismic anisotropy beneath the Northern Apennines (Italy) and its tectonic implications. Geophys. Res. Lett., 23:2721-2724. 
Martynov, G. V., Vernon, F. L., Mellors, R. J., and Pavlis, G. L. (1999). High-frequency attenuation in the crust and upper mantle of the northern Tien Shan. Bull. seism. Soc. Am., 89:215-238.

Mayeda, K., Koynagi, S., Hoshiba, M., Aki, K., and Zeng, Y. (1992). A comparative study of scattering, intrinsic and coda $\mathrm{q} 1$ for Hawaii, Long Valley, and central California between 1.5 and 15 hz. J. Geoph. Res., 97:6643-6659.

Menke, W. (1989). Geophysical Data Analysis: Discrete Inverse Theory. International Geophysics Series. Academic Press.

Miller, V. and Savage, M. (2001). Changes in seismic anisotropy after volcanic eruptions: evidence from Mount Ruapehu. Science, 293:2231-2233.

Nur, A. and Simmons, G. (1969). Stress-induced velocity anisotropy in rock: an experimental study. J. Geophys. Res., 74:6667-6674.

Oth, A. (2007). Source Processes and Spectral Ground Motion Models of IntermediateDepth Vrancea (Romania)Earthquakes. PhD thesis, Fakultat fur Physik, Universitat Karlsruhe (TH), Germany.

Oth, A., Bindi, D., Parolai, S., and Wenzel, F. (2008). S-wave attenuation characteristics beneath the Vrancea region in Romania: New insights from the inversion of ground motion spectra. Bull. Seism. Soc. Am., 98:2482-2497.

Oth, A., Parolai, S., Bindi, D., and Wenzel, F. (2009). Source spectra and site response from $\mathrm{S}$ wave of intermediate-depth Vrancea, Romania, earthquakes. Bull. Seism. Soc. Am., 99:235-254.

Polatidis, A., Kiratzi, A., Hatzidimitriou, P., and Margaris, B. (2003). Attenuation of shear-waves in the back-arc region of the Hellenic arc for frequencies from 0.6 to $16 \mathrm{hz}$. Tectonophysics, 367:29-40. 
Reyners, M., Eberhart-Phillips, D., and Stuart, G. (2007). The role of fluids in lower-crustal earthquakes near continental rifts. Nature, 446:1075-1078.

Reyners, M., Eberhart-Phillips, D., Stuart, G., and Nishimura, Y. (2006). Imaging subduction from the trench to $300 \mathrm{~km}$ depth beneath the central North Island, New Zealand, with Vp and Vp/Vs. Geophys. J. Int., 165:565-583.

Rowlands, D. P., White, R. S., and Haines, A. J. (2005). Seismic tomography of the Tongariro Volcanic Centre, New Zealand. Geophys. J. Int., 163:1180-1194.

Salazar, W., Sardina, V., and de Cortina, J. (2007). A hybrid inversion technique for the evaluation of source, path and site effect employing S-wave spectra for subduction and upper-crustal earthquakes in El Salvador. Bull. Seism. Soc. Am., 97:208-221.

Salmon, M. L. (2008). Crust and upper mantle inhomogeities beneath western North Island, New Zealand: evidence from seimological and electromagnetic data. $\mathrm{PhD}$ thesis, School of Geography, Enviromental and Earth Sciences, Victoria University of Wellington, Wellington, New Zealand.

Sato, H. (1977). Energy propagation including scattering effect; single isotropic scattering. J. Phys. Earth., 25:27-41.

Savage, M. K. (1999). Seismic anisotropy and mantle deformation: What have we learned from shear wave splitting? Rev. Geophys., 37:65-106.

Sherburn, S., Bryan, C. J., Hurst, A. W., Latter, J. H., and Scott, B. J. (1999). Seismicity of Ruapehu volcano, New Zealand, 19711996: a review. J. Volcanol. Geoth. Res., 88:255278.

Sheriff, R. F. and Geldart, L. P. (1995). Exploration Seismology. Cambride University Press.

Smith, W. H. F. and Sandwell, D. T. (1997). Global seafloor topography from satellite altimetry and ship depth soundings. Science, 277:195-196. 
Stein, S. and Wysession, M. (2003). An introduction to seismology, earthquakes and earth structure. Blackwell Publishing, Oxford.

Stern, T. and Davey, F. (1987). A seismic investigation of crustal and upper mantle structure within the Central Volcanic Region of New Zealand. New Zealand Journal of Geology and Geophysics, 30:217-231.

Stern, T., Stratford, W., Seward, A., Henderson, M., Savage, M., Smith, E., Benson, A., Greve, S., and Salmon, M. (2010). Crustmantle structure of the central North Island, New Zealand, based on seismological observations. Geophys. J. Int., 190:58-74.

Stern, T. A. (2009). Reconciling short- and long-term measures of extension in continental back arcs: heat flux, crustal structure and rotations within central North Island, New Zealand. 321:73-87.

Stern, T. A., Stratford, W. R., and Salmon, M. L. (2006). Subduction evolution and mantle dynamics at a continental margin; central North Island, New Zealand. Rev. Geophys, 44:RG4002.

Stratford, W. R. and Stern, T. A. (2004). Strong seismic reflections and melts in the mantle of a continental back-arc basin. Geophys. Res. Lett., 31:L06622.

Stratford, W. R. and Stern, T. A. (2006). Crustal and upper mantle structure of a continental back-arc: Central North Island, New Zealand. Geophys. J. Int., 166:469-484.

Styles, K. E. (2009). Shear wave attenuation structure and anisotropy in the Hikurangi subduction zone, central North Island, New Zealand. PhD thesis, School of Earth and Environment, The University of Leeds, England.

Sutherland, R., Berryman, K., and Norris, R. (2006). Quaternary slip rates and geomorphology of the Alpine Fault: Implications for kinematics and seismic hazard in southwest New Zealand. Geological Society of America Bulletin, 118:464-474. 
Tao, G. and King, M. S. (1990). Shear-wave velocity and Q anisotropy in rocks: a loboratory study. Int. J. Rock. Mech. Min. Sci., 27:353-361.

Titzschkau, T., Savage, M., and Hurst, T. (2009). Changes in attenuation related to eruptions of Mt. Ruapehu Volcano, New Zealand. J. Volcanol. Geoth. Res., Article in press.

Toksoz, M. N. and Johnston, D. H. (1981). Seismic wave attenuation. Geophysics reprint series, No. 2. Society of Exploration Geophysicist.

Villa, J., Correig, A. M., and Carbonell, R. (1997). New evidence of azimuthal variation at Campi Flegrei: Attenuation and predominant frequency. Pure Appl. Geophys, 150:283304.

Wilson, C. J. N., Houghton, B. F., McWilliams, M. O., Lanphere, M. A., Weaver, S. D., and Briggs, R. M. (1995). Volcanic and structural evolution of Taupo Volcanic Zone, New Zealand: a review. J. Volcanol. Geoth. Res., 68:1-28.

Wustefeld, A., Bokelmann, G., Zaroli, C., and Barruol, G. (2008). Splitlab: A shear-wave splitting environment in Matlab. Computers and Geosciences, 34:515-528.

Yu, W. C. and Wen, L. X. (2006). Inner core attenuation anisotropy. Earth Planet. Sci. Lett, 245:581-594. 


\section{Appendix A}

\section{Event information}

Table A.1 Events used for Q model of inside TVZ

\begin{tabular}{|c|l|l|l|l|l|}
\hline Cupsid & Evt date & Evt lat & Evt lon & Evt depth & Evt mag \\
\hline 2283711 & 2004234 & -38.99 & 175.72 & 5.51 & 2.65 \\
2283569 & 2004234 & -39.00 & 175.71 & 5.22 & 2.01 \\
2285465 & 2004238 & -38.99 & 175.71 & 5.00 & 2.49 \\
2285516 & 2004238 & -38.99 & 175.71 & 4.20 & 2.44 \\
2290416 & 2004250 & -38.84 & 175.86 & 5.00 & 2.86 \\
2485053 & 2004294 & -39.13 & 175.66 & 4.77 & 2.24 \\
2342078 & 2004357 & -38.79 & 175.67 & 5.00 & 2.23 \\
2558323 & 2005022 & -38.90 & 175.87 & 4.21 & 2.20 \\
2376709 & 2005073 & -39.02 & 175.73 & 5.00 & 2.80 \\
2403552 & 2005133 & -38.83 & 175.82 & 5.00 & 3.42 \\
2469742 & 2005280 & -39.27 & 175.55 & 3.48 & 2.24 \\
2473535 & 2005289 & -38.85 & 175.69 & 5.00 & 2.30 \\
2494762 & 2005336 & -39.13 & 175.67 & 3.79 & 2.43 \\
2507731 & 2006002 & -39.00 & 175.78 & 7.31 & 2.67 \\
2507784 & 2006002 & -39.00 & 175.78 & 9.48 & 2.15 \\
2517145 & 2006023 & -39.00 & 175.78 & 5.00 & 2.25 \\
2517146 & 2006023 & -39.00 & 175.78 & 7.66 & 2.52 \\
2517149 & 2006023 & -38.99 & 175.78 & 10.05 & 2.11 \\
2532957 & 2006061 & -38.92 & 175.89 & 8.67 & 2.11 \\
2554479 & 2006106 & -38.88 & 175.64 & 5.00 & 2.49 \\
2562153 & 2006122 & -39.14 & 175.64 & 4.69 & 2.07 \\
2568736 & 2006136 & -38.98 & 175.81 & 7.59 & 2.23 \\
2593778 & 2006190 & -38.96 & 175.71 & 11.08 & 2.23 \\
2593780 & 2006190 & -38.96 & 175.71 & 8.73 & 2.13 \\
2608767 & 2006222 & -39.01 & 175.75 & 5.20 & 2.65 \\
2608774 & 2006222 & -39.01 & 175.75 & 5.00 & 2.38 \\
\hline & & & & Continued on next page \\
\hline
\end{tabular}


Table A.1 - continued from previous page

\begin{tabular}{|c|c|c|c|c|c|}
\hline Cupsid & Evt date & Evt lat & Evt lon & Evt depth & Evt mag \\
\hline 2608778 & 2006222 & -39.01 & 175.75 & 9.99 & 2.01 \\
\hline 2619218 & 2006245 & -39.01 & 175.74 & 7.85 & 2.28 \\
\hline 2630946 & 2006270 & -38.93 & 175.72 & 7.95 & 2.38 \\
\hline 2631017 & 2006271 & -38.92 & 175.71 & 5.00 & 2.17 \\
\hline 2657786 & 2006327 & -38.97 & 175.77 & 6.37 & 2.28 \\
\hline 2661705 & 2006335 & -38.99 & 175.81 & 5.00 & 2.81 \\
\hline 2662040 & 2006336 & -38.99 & 175.82 & 6.62 & 2.14 \\
\hline 2680142 & 2007009 & -38.97 & 175.82 & 6.95 & 2.26 \\
\hline 2704153 & 2007061 & -38.97 & 175.82 & 6.32 & 2.10 \\
\hline 2712064 & 2007077 & -39.02 & 175.76 & 5.00 & 2.04 \\
\hline 2717771 & 2007089 & -38.94 & 175.74 & 5.00 & 2.87 \\
\hline 2718437 & 2007091 & -38.89 & 175.80 & 5.60 & 2.14 \\
\hline 2722077 & 2007098 & -38.94 & 175.88 & 6.82 & 2.22 \\
\hline 2771473 & 2007207 & -38.93 & 175.84 & 5.00 & 2.70 \\
\hline 2771475 & 2007207 & -38.93 & 175.85 & 5.00 & 2.14 \\
\hline 2771553 & 2007207 & -38.94 & 175.79 & 2.57 & 3.11 \\
\hline 2771378 & 2007207 & -38.93 & 175.85 & 5.67 & 2.33 \\
\hline 2771402 & 2007207 & -38.92 & 175.82 & 5.00 & 2.63 \\
\hline 2771980 & 2007208 & -38.93 & 175.85 & 5.00 & 2.48 \\
\hline 2772028 & 2007208 & -38.93 & 175.83 & 5.28 & 2.35 \\
\hline 2772064 & 2007208 & -38.93 & 175.86 & 5.94 & 2.93 \\
\hline 2772336 & 2007209 & -38.94 & 175.85 & 7.12 & 2.02 \\
\hline 2797040 & 2007264 & -39.04 & 175.79 & 10.02 & 2.59 \\
\hline 2819924 & 2007314 & -39.12 & 175.65 & 4.28 & 2.03 \\
\hline 2823239 & 2007320 & -38.91 & 175.86 & 7.46 & 2.34 \\
\hline 2831557 & 2007339 & -39.13 & 175.66 & 5.00 & 2.01 \\
\hline 2844837 & 2008001 & -38.99 & 175.75 & 5.57 & 2.19 \\
\hline 2852376 & 2008018 & -39.10 & 175.67 & 3.00 & 3.72 \\
\hline 2852375 & 2008018 & -39.11 & 175.69 & 3.00 & 3.07 \\
\hline 2855969 & 2008025 & -39.07 & 175.66 & 7.21 & 2.02 \\
\hline 2855673 & 2008025 & -39.08 & 175.68 & 5.97 & 2.32 \\
\hline 2855797 & 2008025 & -39.08 & 175.68 & 5.00 & 2.36 \\
\hline 2867955 & 2008050 & -39.04 & 175.79 & 7.94 & 2.22 \\
\hline 2880771 & 2008076 & -39.14 & 175.64 & 3.17 & 2.14 \\
\hline 2881694 & 2008078 & -39.06 & 175.73 & 5.00 & 2.38 \\
\hline 2887121 & 2008090 & -38.98 & 175.66 & 5.26 & 2.34 \\
\hline 2888474 & 2008093 & -38.98 & 175.72 & 7.09 & 2.47 \\
\hline 2899045 & 2008115 & -39.01 & 175.72 & 1.83 & 2.13 \\
\hline 2915807 & 2008151 & -39.02 & 175.81 & 3.66 & 3.37 \\
\hline 2915902 & 2008151 & -39.01 & 175.80 & 5.00 & 2.03 \\
\hline 2918368 & 2008156 & -39.02 & 175.82 & 3.00 & 2.24 \\
\hline 2918478 & 2008157 & -39.03 & 175.81 & 3.00 & 2.51 \\
\hline 2918616 & 2008157 & -38.78 & 175.86 & 2.76 & 2.26 \\
\hline
\end{tabular}


Table A.1 - continued from previous page

\begin{tabular}{|c|l|l|l|l|l|}
\hline Cupsid & Evt date & Evt lat & Evt lon & Evt depth & Evt mag \\
\hline 2933865 & 2008187 & -38.96 & 175.72 & 4.28 & 2.02 \\
2935261 & 2008190 & -39.02 & 175.75 & 6.05 & 2.13 \\
2938750 & 2008197 & -38.93 & 175.82 & 5.00 & 2.13 \\
2944198 & 2008208 & -39.01 & 175.74 & 12.00 & 2.17 \\
2945700 & 2008211 & -38.94 & 175.71 & 5.00 & 2.48 \\
2945663 & 2008211 & -38.94 & 175.71 & 5.00 & 2.62 \\
2945864 & 2008212 & -38.94 & 175.71 & 5.00 & 2.17 \\
2946956 & 2008214 & -38.94 & 175.71 & 5.00 & 2.15 \\
2949560 & 2008219 & -38.95 & 175.70 & 5.00 & 2.04 \\
2951293 & 2008223 & -38.84 & 175.88 & 5.00 & 2.52 \\
2952218 & 2008225 & -38.84 & 175.85 & 5.00 & 2.46 \\
2957115 & 2008235 & -39.04 & 175.68 & 5.00 & 2.40 \\
2957361 & 2008235 & -39.04 & 175.69 & 5.00 & 2.01 \\
2961706 & 2008244 & -38.84 & 175.92 & 5.00 & 2.30 \\
3024650 & 2009003 & -38.89 & 175.88 & 5.00 & 2.14 \\
3029757 & 2009013 & -38.98 & 175.72 & 5.97 & 2.08 \\
3030322 & 2009014 & -39.08 & 175.68 & 5.00 & 3.12 \\
3030120 & 2009014 & -38.86 & 175.87 & 5.00 & 2.02 \\
3030339 & 2009014 & -39.08 & 175.68 & 5.00 & 2.35 \\
3036573 & 2009027 & -39.08 & 175.76 & 5.00 & 2.28 \\
3037129 & 2009028 & -39.10 & 175.65 & 5.00 & 2.31 \\
\hline
\end{tabular}

Table A.2 Event used for Q model of outside TVZ

\begin{tabular}{|c|l|l|l|l|l|}
\hline Cupsid & Evt date & Evt lat & Evt lon & Evt depth & Evt mag \\
\hline 2223442 & 2004122 & -39.24 & 175.32 & 15.65 & 2.32 \\
2226361 & 2004127 & -39.31 & 175.29 & 5.00 & 2.23 \\
2226627 & 2004128 & -39.31 & 175.33 & 12.00 & 3.07 \\
2226865 & 2004128 & -39.30 & 175.33 & 12.00 & 2.30 \\
2252384 & 2004175 & -39.26 & 175.30 & 22.24 & 2.20 \\
2258972 & 2004187 & -39.25 & 175.29 & 16.08 & 2.04 \\
2258443 & 2004187 & -39.29 & 175.46 & 13.18 & 2.01 \\
2264285 & 2004195 & -39.40 & 175.29 & 8.50 & 2.06 \\
2268180 & 2004203 & -39.23 & 175.32 & 13.20 & 2.19 \\
2269167 & 2004205 & -39.35 & 175.38 & 16.28 & 2.06 \\
2273155 & 2004214 & -39.35 & 175.41 & 13.97 & 2.36 \\
2276848 & 2004220 & -39.32 & 175.52 & 13.11 & 2.13 \\
2288218 & 2004244 & -39.31 & 175.35 & 30.88 & 2.33 \\
2295430 & 2004261 & -39.26 & 175.47 & 15.72 & 2.09 \\
2297938 & 2004266 & -39.22 & 175.31 & 26.01 & 2.01 \\
2301462 & 2004274 & -39.25 & 175.25 & 13.45 & 2.27 \\
\hline \multicolumn{7}{|c|}{ Continued on next page } \\
\hline
\end{tabular}


Table A.2 - continued from previous page

\begin{tabular}{|c|c|c|c|c|c|}
\hline Cupsid & Evt date & Evt lat & Evt lon & Evt depth & Evt mag \\
\hline 2301796 & 2004275 & -39.27 & 175.44 & 17.99 & 2.18 \\
\hline 2301893 & 2004275 & -39.24 & 175.25 & 10.60 & 2.72 \\
\hline 2302302 & 2004276 & -39.24 & 175.33 & 14.29 & 2.06 \\
\hline 2304574 & 2004281 & -39.24 & 175.32 & 19.49 & 2.10 \\
\hline 2314788 & 2004304 & -39.25 & 175.25 & 13.59 & 2.30 \\
\hline 2329743 & 2004334 & -39.23 & 175.33 & 14.34 & 2.27 \\
\hline 2522919 & 2004343 & -39.42 & 175.41 & 5.00 & 2.33 \\
\hline 2345765 & 2005001 & -39.27 & 175.44 & 12.86 & 2.74 \\
\hline 2345770 & 2005001 & -39.30 & 175.41 & 12.00 & 2.01 \\
\hline 2355970 & 2005024 & -39.23 & 175.48 & 12.40 & 2.80 \\
\hline 2361176 & 2005036 & -39.24 & 175.47 & 14.22 & 2.13 \\
\hline 2368977 & 2005055 & -39.23 & 175.47 & 14.10 & 2.09 \\
\hline 2370815 & 2005059 & -39.23 & 175.45 & 14.46 & 2.06 \\
\hline 2594369 & 2005070 & -39.41 & 175.52 & 5.00 & 2.05 \\
\hline 2378454 & 2005077 & -39.26 & 175.21 & 28.80 & 2.72 \\
\hline 2380251 & 2005081 & -39.22 & 175.39 & 9.07 & 2.62 \\
\hline 2383016 & 2005087 & -39.24 & 175.32 & 13.19 & 2.79 \\
\hline 2385407 & 2005093 & -39.23 & 175.32 & 13.62 & 2.55 \\
\hline 2390123 & 2005104 & -39.25 & 175.22 & 13.17 & 2.01 \\
\hline 2617848 & 2005107 & -39.41 & 175.73 & 12.41 & 2.64 \\
\hline 2392562 & 2005109 & -39.37 & 175.60 & 8.91 & 2.27 \\
\hline 2393711 & 2005111 & -39.28 & 175.20 & 11.44 & 2.25 \\
\hline 2394291 & 2005113 & -39.28 & 175.23 & 21.94 & 2.51 \\
\hline 2394458 & 2005113 & -39.28 & 175.24 & 24.02 & 2.39 \\
\hline 2396978 & 2005118 & -39.24 & 175.33 & 13.92 & 2.31 \\
\hline 2401380 & 2005128 & -39.23 & 175.42 & 9.64 & 2.12 \\
\hline 2401616 & 2005129 & -39.28 & 175.20 & 10.87 & 2.21 \\
\hline 2403591 & 2005133 & -39.26 & 175.23 & 15.47 & 2.44 \\
\hline 2405968 & 2005138 & -39.25 & 175.28 & 14.53 & 2.46 \\
\hline 2428115 & 2005187 & -39.34 & 175.57 & 17.65 & 2.12 \\
\hline 2428845 & 2005188 & -39.31 & 175.30 & 28.71 & 2.26 \\
\hline 2432753 & 2005196 & -39.33 & 175.49 & 10.70 & 2.41 \\
\hline 2434827 & 2005200 & -39.38 & 175.53 & 10.14 & 2.59 \\
\hline 2435569 & 2005202 & -39.37 & 175.54 & 6.70 & 2.20 \\
\hline 2436195 & 2005203 & -39.35 & 175.54 & 3.53 & 2.38 \\
\hline 2439273 & 2005210 & -39.24 & 175.33 & 13.54 & 2.69 \\
\hline 2450013 & 2005235 & -39.34 & 175.32 & 29.96 & 2.07 \\
\hline 2451974 & 2005239 & -39.27 & 175.35 & 12.00 & 2.02 \\
\hline 2452439 & 2005240 & -39.35 & 175.55 & 1.54 & 2.11 \\
\hline 2452440 & 2005240 & -39.42 & 175.58 & 12.37 & 2.21 \\
\hline 2454018 & 2005244 & -39.24 & 175.32 & 17.82 & 2.05 \\
\hline 2455947 & 2005248 & -39.31 & 175.29 & 12.00 & 2.07 \\
\hline 2459027 & 2005255 & -39.25 & 175.39 & 8.46 & 2.06 \\
\hline
\end{tabular}


Table A.2 - continued from previous page

\begin{tabular}{|c|l|l|l|l|l|}
\hline Cupsid & Evt date & Evt lat & Evt lon & Evt depth & Evt mag \\
\hline 2464712 & 2005269 & -39.24 & 175.23 & 23.62 & 2.49 \\
2476484 & 2005295 & -39.23 & 175.34 & 14.85 & 2.31 \\
2476751 & 2005296 & -39.24 & 175.34 & 14.24 & 2.23 \\
2480193 & 2005304 & -39.26 & 175.46 & 15.19 & 2.02 \\
2483009 & 2005310 & -39.24 & 175.49 & 7.30 & 2.18 \\
2487566 & 2005320 & -39.35 & 175.40 & 16.72 & 2.18 \\
2506485 & 2005364 & -39.24 & 175.32 & 16.96 & 2.22 \\
\hline
\end{tabular}




\section{Appendix B}

\section{Other decay curves}




\section{B.1 The TVZ decay functions}
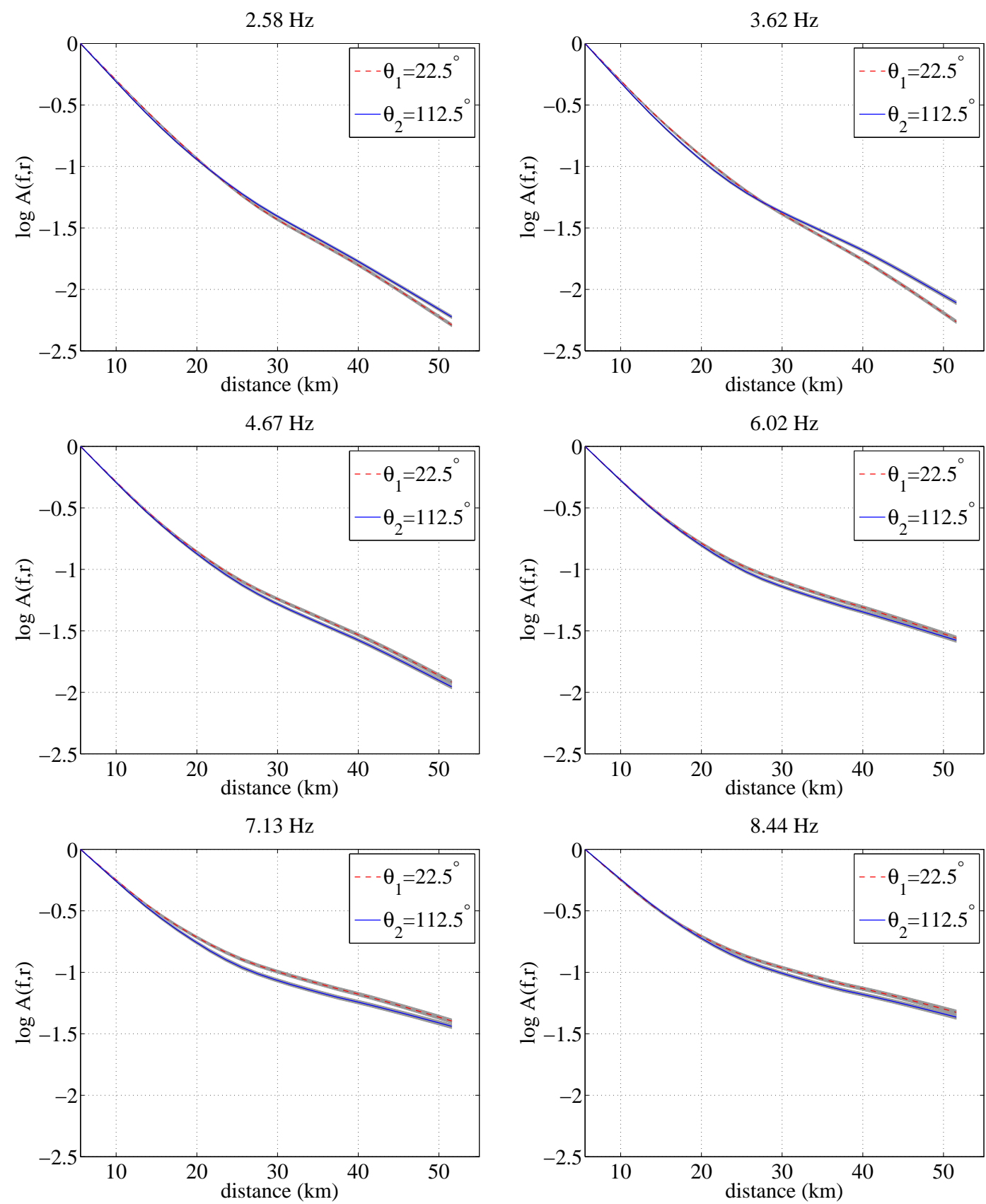

Figure B.1 Amplitude decay functions versus hypocentral distance for rotation $22.5^{\circ}$ at six frequencies obtained from the TVZ data. The dashed red and blue lines represent the mean of 200 bootstrap samples and the gray shaded area represents the mean $\pm 2 \sigma$. 

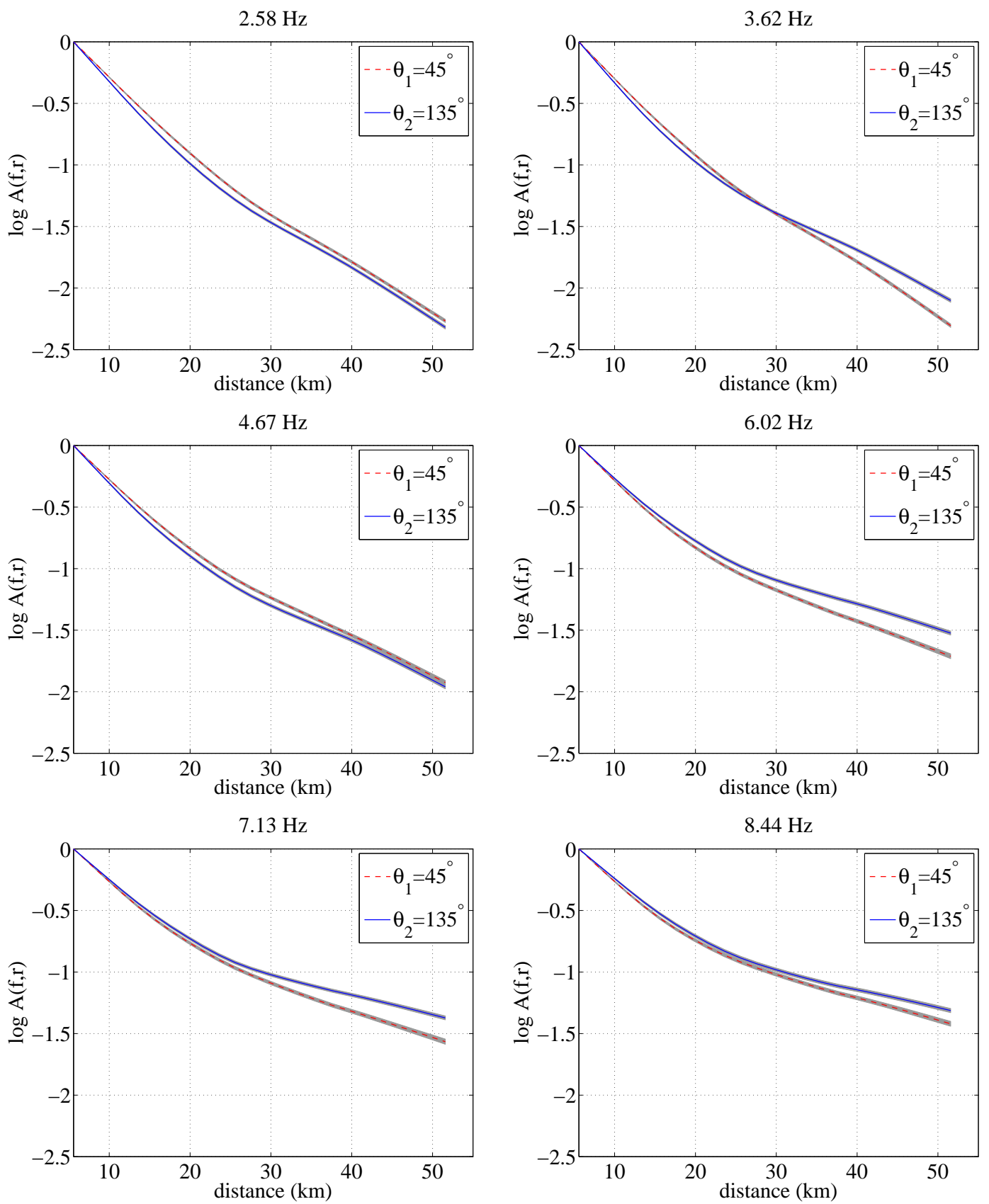

Figure B.2 Amplitude decay functions versus hypocentral distance for rotation $45^{\circ}$ at six frequencies obtained from the TVZ data. The dashed red and blue lines represent the mean of 200 bootstrap samples and the gray shaded area represents the mean $\pm 2 \sigma$. 

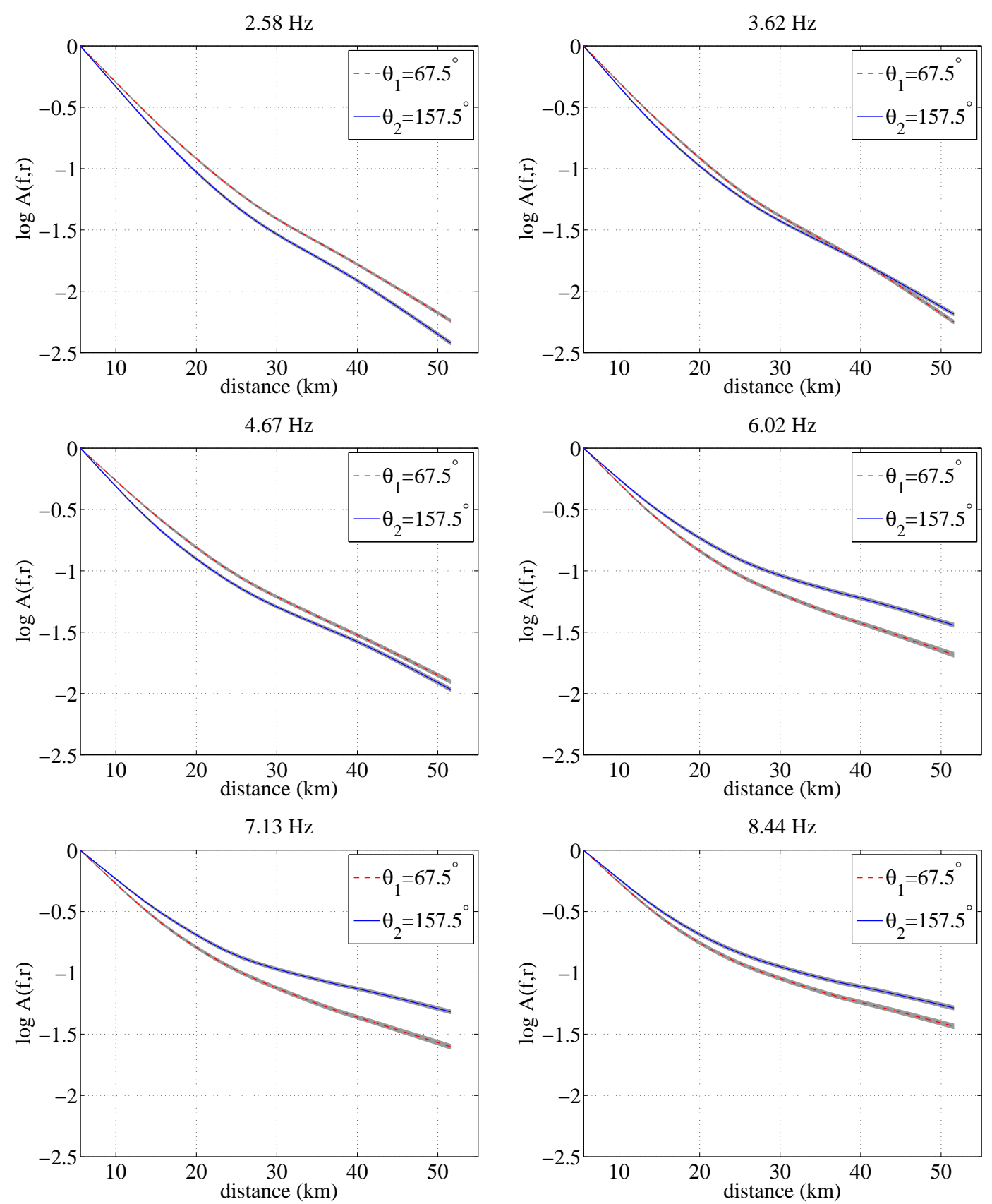

Figure B.3 Amplitude decay functions versus hypocentral distance for rotation $67.5^{\circ}$ at six frequencies obtained from the TVZ data. The dashed red and blue lines represent the mean of 200 bootstrap samples and the gray shaded area represents the mean $\pm 2 \sigma$. 


\section{B.2 The non-TVZ decay functions}
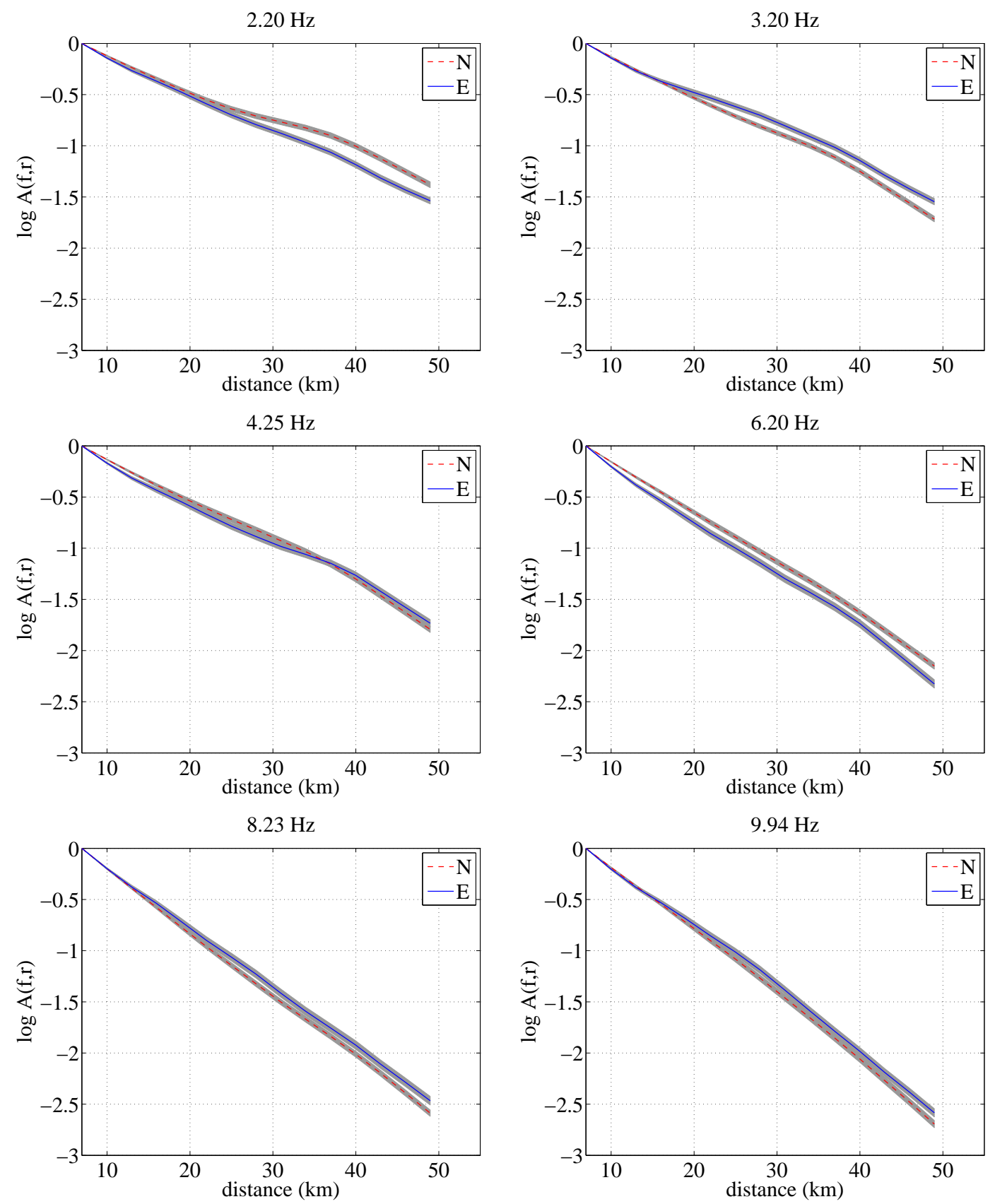

Figure B.4 Amplitude decay functions versus hypocentral distance for $\mathrm{E}$ and $\mathrm{N}$ components at six frequencies obtained from the non-TVZ data. The dashed red and blue lines represent the mean of 200 bootstrap samples and the gray shaded area represents the mean $\pm 2 \sigma$. 

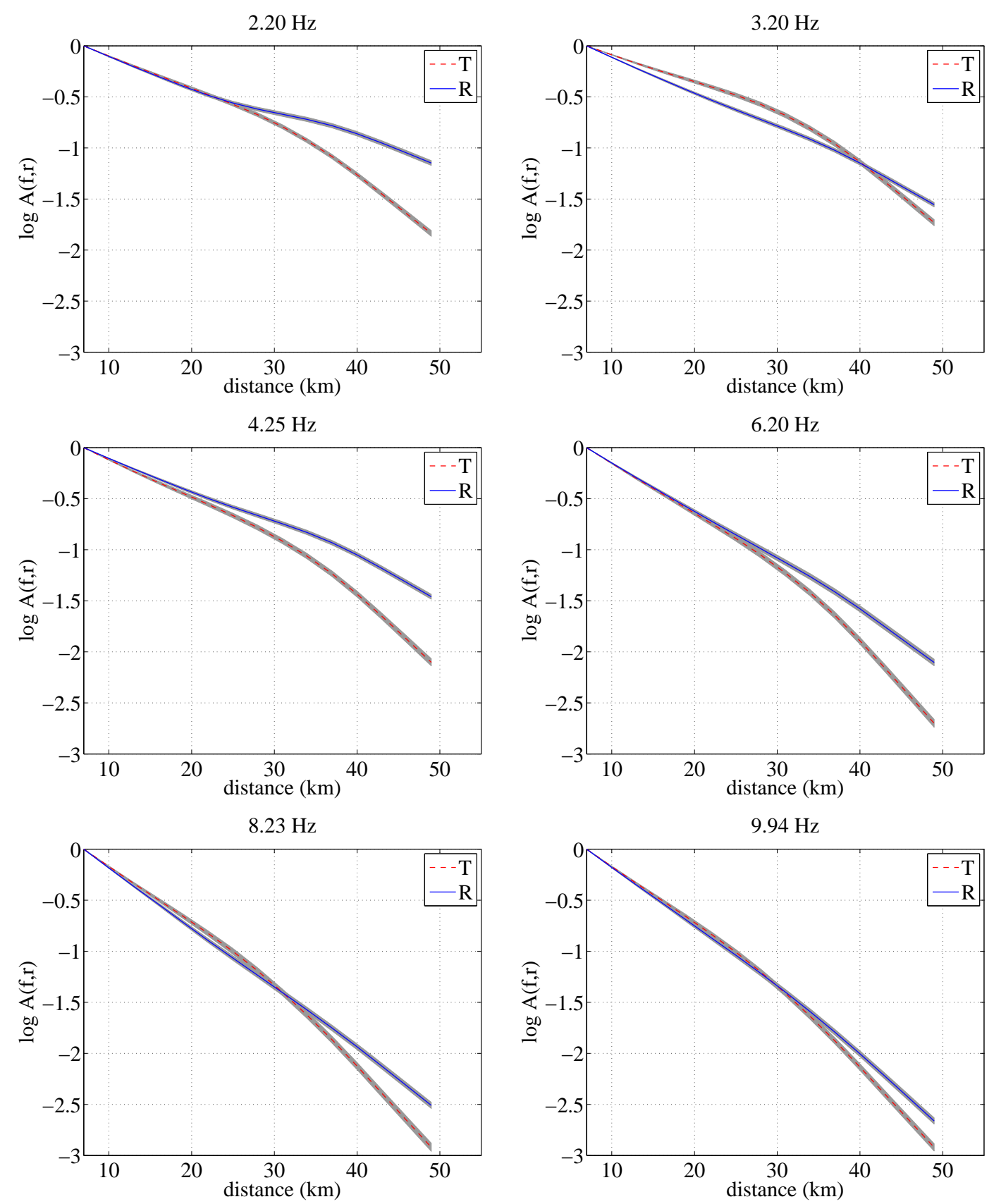

Figure B.5 Amplitude decay functions versus hypocentral distance for R and $\mathrm{T}$ components at six frequencies obtained from the non-TVZ data. The dashed red and blue lines represent the mean of 200 bootstrap samples and the gray shaded area represents the mean $\pm 2 \sigma$. 

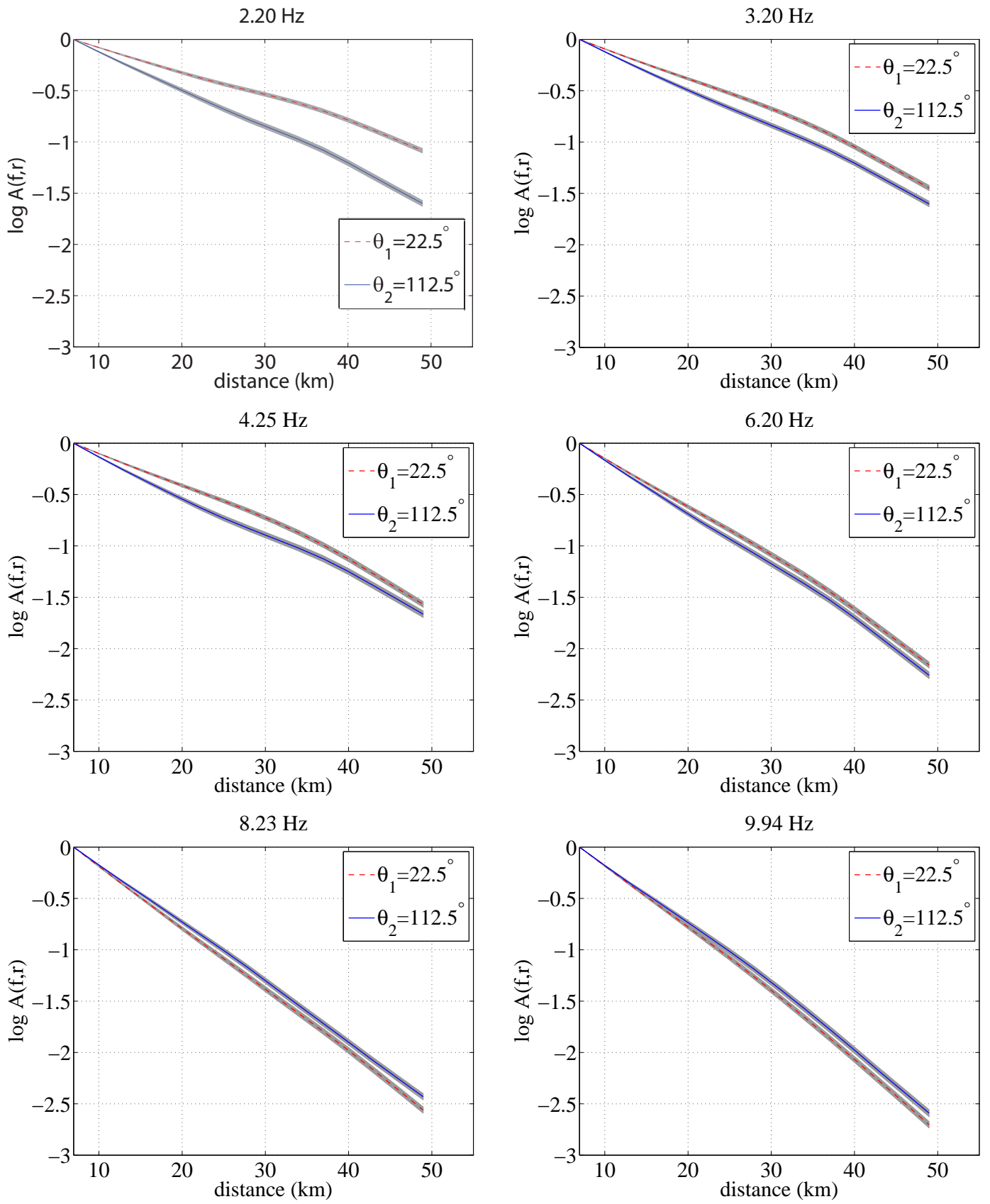

Figure B.6 Amplitude decay functions versus hypocentral distance for rotation $22.5^{\circ}$ at six frequencies obtained from the non-TVZ data. The dashed red and blue lines represent the mean of 200 bootstrap samples and the gray shaded area represents the mean $\pm 2 \sigma$. 

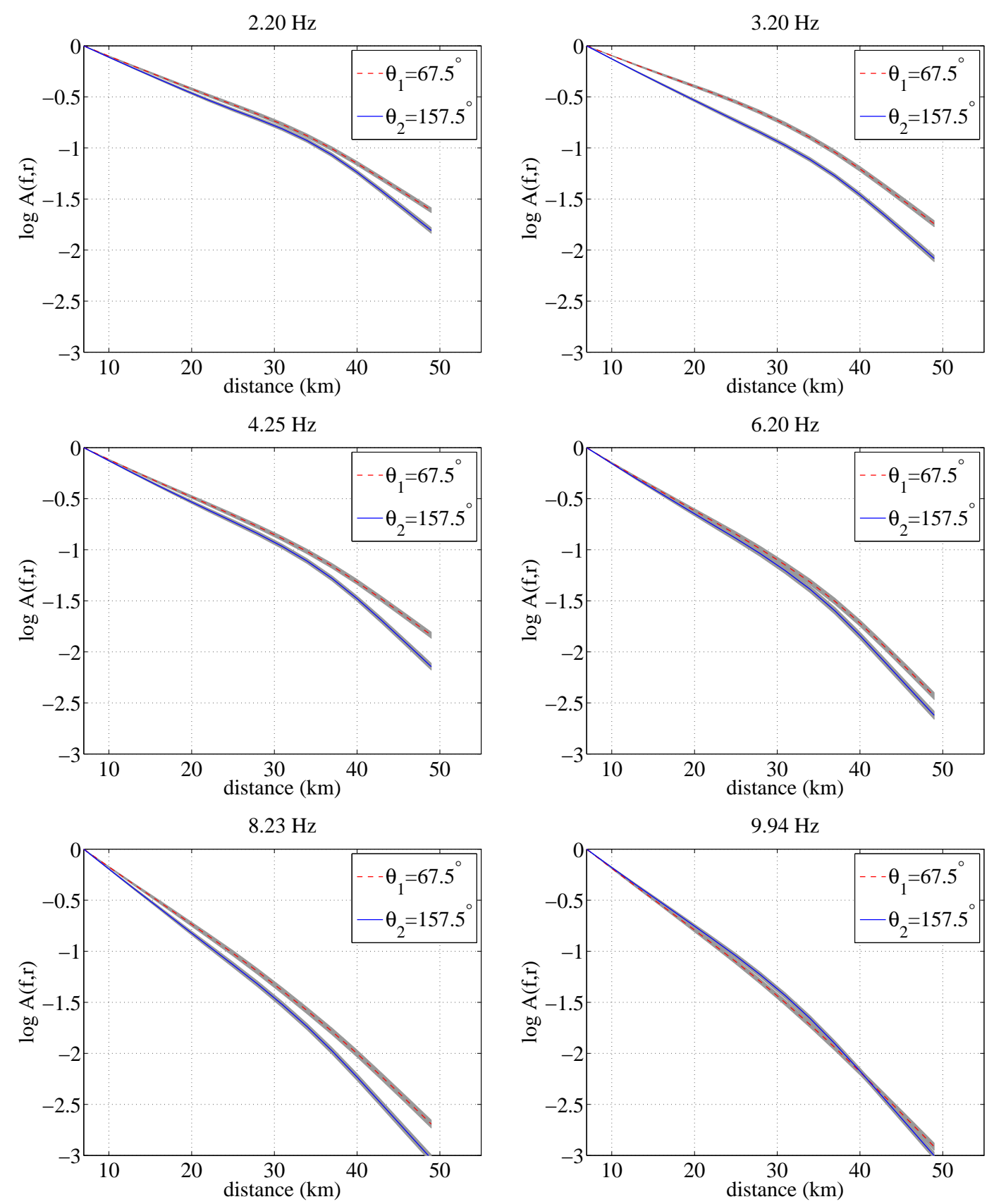

Figure B.7 Amplitude decay functions versus hypocentral distance for rotation $67.5^{\circ}$ at six frequencies obtained from the non-TVZ data. The dashed red and blue lines represent the mean of 200 bootstrap samples and the gray shaded area represents the mean $\pm 2 \sigma$. 


\section{Appendix C}

\section{Spectral ratio-differential attenuation method}

\section{C.1 Spectral ratio-differential attenuation method}

In the initial stages of this study, we implemented the differential attenuation method proposed by Carter and Kendall (2006) to quantify attenuation anisotropy. The results obtained using this approach are discussed in Appendix C.2. The differential attenuation method compares the relative frequency content of fast $\left(S_{1}\right)$ and slow $\left(S_{2}\right)$ shear-waves (Carter and Kendall, 2006), and is based on the fact that the amplitude decreases with increasing frequency. Thus, forming the ratio of spectral amplitudes for $S 1$ and $S 2$ yields the dimensionless differential attenuation $\Delta Q_{f}^{-1}$.

The amplitude spectra of $S 1$ and $S 2$ recorded at a seismic station as a function of frequency satisfy

$$
A_{S n}(f)=G_{S n}(f) S_{S n}(f) R_{S n}(f) \exp \left[\frac{-\pi t_{S n} f}{Q_{S n}}\right], \quad n=1,2
$$

where $G_{S n}$ is the geometrical spreading factor experienced by $S_{1}$ and $S_{2}, S_{S n}$ is the source spectrum, $R_{S n}$ is the instrument response, $t_{S n}$ is the travel time of the split shear waves and $Q_{S n}$ describes the frequency-independent quality factor of the split waves. The spectral ratio method (Bath, 1974) can then be used to determine the relative attenuation between the waveforms. Spectral division of $A_{S 1}$ with $A_{S 2}$ is expressed as,

$$
\frac{A_{S 1}(f)}{A_{S 2}(f)}=\frac{G_{S 1}(f) S_{S 1}(f) R_{S 1}(f)}{G_{S 2}(f) S_{S 2}(f) R_{S 2}(f)} \exp \left[\pi f\left(\frac{t_{S 2}}{Q_{S 2}}-\frac{t_{S 1}}{Q_{S 1}}\right)\right]
$$


Because both $S_{1}$ and $S_{2}$ recordings are from the same source and recorded by the same station, we assume that $S_{S 1}(f)=S_{S 2}(f)$ and $R_{S 1}(f)=R_{S 2}(f)$, but we check the instrument response and correct for any variation if they are not equal. Therefore, we can neglect the source spectrum and the receiver response. We further assume that the geometric spreading factors $\left(G_{S 1}(f)\right.$ and $\left.G_{S 2}(f)\right)$ have the same frequency dependence; $\frac{G_{S 1}(f)}{G_{S 2}(f)}$ is then independent of frequency. Taking logarithms on both sides of eq. C.2, which allows us to determine the corner frequency of the spectrum, we obtain

$$
\log \left[\frac{A_{S 1}(f)}{A_{S 2}(f)}\right]=c+\log \exp \left[\pi f\left(\frac{t_{S 2}}{Q_{S 2}}-\frac{t_{S 1}}{Q_{S 1}}\right)\right]
$$

The first term on the right hand side of eq. C.3 is independent of frequency $f$ in the $G_{S n}$ term in equation eq. C.1. Therefore, a plot of $\log \left[\frac{A_{S 1}(f)}{A_{S 2}(f)}\right]$ versus $f$ yields a gradient $m$, from which the differential attenuation term $\Delta Q_{f}^{-1}$ can be calculated after dividing $\left(\log _{e} 10 . m\right)$ by $\pi t_{S 1} ;$ In other words,

$$
\Delta Q_{f}^{-1}=\frac{t_{S 2}}{t_{S 1} Q_{S 2}}-\frac{1}{Q_{S 1}}
$$

We use the same seismograms as analysed using the non-parametric GIT method. We split the $S$-wave into $S_{1}$ and $S_{2}$ using a modified Splitlab code (Wustefeld et al., 2008). In calculating the spectral ratio, a time window and pre-event noise are chosen using the same window lengths. Amplitude spectra are then calculated through fast Fourier transform and smoothed using the Konno-Ohmachi windowing function (Konno and Omachi, 1998) as outlined in Section 3.2.1. To calculate the differential attenuation, the signals are processed to identify an appropriate bandwidth by comparing the amplitude spectra for $S_{1}$ and $S_{2}$ with the pre-event spectral amplitude of the noise (see Figure C.1).

\section{C.2 Results}

We display the results obtained using the spectral ratio method of Carter and Kendall (2006) (see Section C.1 for details of the method). We analyse the same dataset as the 
non-parametric GIT method, which forms the bulk of Chapter 4, in order to determine the preferred method of quantifying seismic attenuation anisotropy.

The right panel of Fig. C.1 reveals a negative slope of the spectral ratios indicating in this case that the $\mathrm{S}$-wave the fast polarization is more attenuated than that with the slow polarization. The reverse is true for the earthquake depicted in the left panel. The two events exhibit splitting times of 0.16 and $0.2 \mathrm{~s}$. Although the splitting times are slightly different, this should not adversely affect the attenuation anisotropy measurement. The important thing in this method is that the shear wave must be split in order to quantify attenuation anisotropy (Carter and Kendall, 2006). We choose appropriate frequency bands for the spectral ratio by comparing the amplitude spectra of $S_{1}$ and $S_{2}$ with the pre-event spectral amplitudes of noise. Interestingly, the results for event 2 appear to contradict (Hudson, 1981)'s theory, which predicts that the slower arrival $S_{2}$ should be more attenuated in high frequencies than the faster arrival $S_{1}$. However, the results are in good agreement with previous observations reported by Carter and Kendall (2006), who also found some results showing $S_{2}$ to be more attenuated than $S_{1}$ and some with $S_{1}$ more attenuated that $S_{2}$ and they state that the precise mechanism of the effect is still unclear. We show in Section 4.4.2 that polarisation dependent amplification occurs in this region, which could affect the measurements made with this method. 

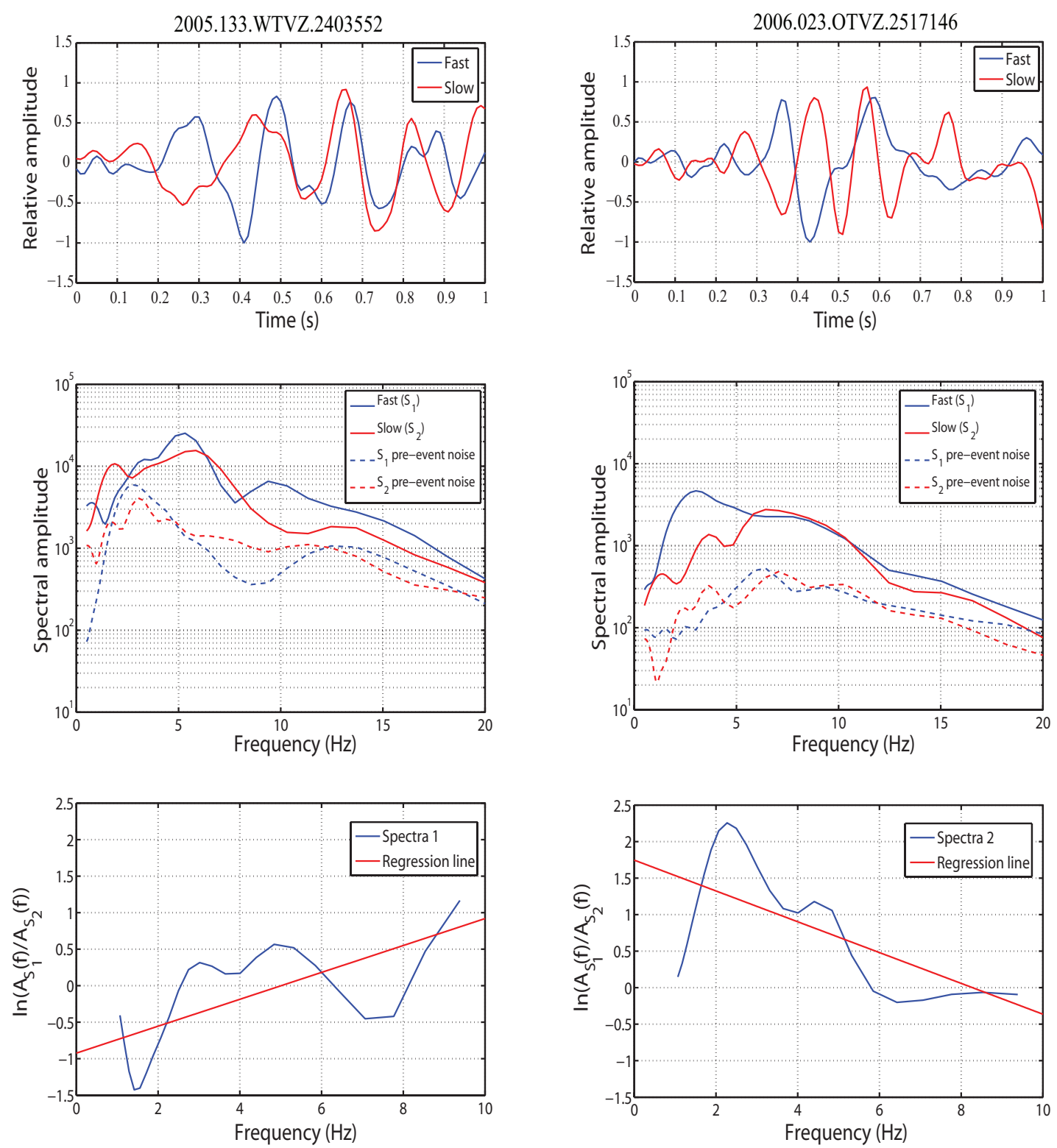

Figure C.1 Result for two earthquakes recorded at GeoNet stations WTVZ and OTVZ, showing the seismograms of the $S_{1}$ and $S_{2}$ components. The top panel shows splitting results. The middle panel shows the spectral amplitudes of $S_{1}$ and $S_{2}$ and their pre-event noise. Data 2 (right) in the bottom panel shows that $S_{2}$ is richer in high frequencies than $S_{1}$, whereas data 1 (left) shows the converse. 